\title{
The Atmospheric Response to High Nonthermal Electron Beam Fluxes in Solar Flares. I. Modeling the Brightest NUV Footpoints in the X1 Solar Flare of 2014 March 29
}

\author{
Adam F. Kowalski ${ }^{1,2,3,4}$, Joel C. Allred ${ }^{4}$, Adrian Daw ${ }^{4}$, Gianna Cauzzi ${ }^{5}$, and Mats Carlsson ${ }^{6}$ \\ ${ }^{1}$ Department of Astrophysical and Planetary Sciences, University of Colorado Boulder, 2000 Colorado Ave, Boulder, CO 80305, USA; \\ Adam.Kowalski@lasp.colorado.edu \\ ${ }^{2}$ National Solar Observatory, University of Colorado Boulder, 3665 Discovery Drive, Boulder, CO 80303, USA \\ ${ }^{3}$ Department of Astronomy, University of Maryland, College Park, MD 20742, USA \\ NASA/Goddard Space Flight Center, Code 671, Greenbelt, MD 20771, USA \\ 5 INAF-Osservatorio Astrofisico di Arcetri, I-50125 Firenze, Italy \\ ${ }^{6}$ Institute of Theoretical Astrophysics, University of Oslo, PO Box 1029 Blindern, NO-0315 Oslo, Norway \\ Received 2016 June 28; revised 2016 September 22; accepted 2016 September 22; published 2017 February 6
}

\begin{abstract}
The 2014 March 29 X1 solar flare (SOL20140329T17:48) produced bright continuum emission in the far- and near-ultraviolet (NUV) and highly asymmetric chromospheric emission lines, providing long-sought constraints on the heating mechanisms of the lower atmosphere in solar flares. We analyze the continuum and emission line data from the Interface Region Imaging Spectrograph (IRIS) of the brightest flaring magnetic footpoints in this flare. We compare the NUV spectra of the brightest pixels to new radiative-hydrodynamic predictions calculated with the RADYN code using constraints on a nonthermal electron beam inferred from the collisional thick-target modeling of hard X-ray data from Reuven Ramaty High Energy Solar Spectroscopic Imager. We show that the atmospheric response to a high beam flux density satisfactorily achieves the observed continuum brightness in the NUV. The NUV continuum emission in this flare is consistent with hydrogen (Balmer) recombination radiation that originates from low optical depth in a dense chromospheric condensation and from the stationary beam-heated layers just below the condensation. A model producing two flaring regions (a condensation and stationary layers) in the lower atmosphere is also consistent with the asymmetric Fe II chromospheric emission line profiles observed in the impulsive phase.
\end{abstract}

Key words: Sun: atmosphere - Sun: flares - radiation: dynamics - radiative transfer

\section{Introduction}

The spectral energy distribution of the ultraviolet, optical, and infrared continuum (white-light) emission contains important information on the atmospheric response at the highest densities in the flare atmosphere but has remained largely unconstrained due to a lack of broad-wavelength spectral observations (Fletcher et al. 2007). The white-light emission provides important constraints on the strength and depth of flare heating resulting from magnetic energy release in the corona. White-light emission is thought to be produced by the energy deposition by nonthermal electrons due to the close spatial and temporal coincidence with hard X-ray emission (e.g., Rust \& Hegwer 1975; Hudson et al. 1992; Metcalf et al. 2003; Martínez Oliveros et al. 2012). The source of these nonthermal electrons is controversial; in the standard flare model, they are accelerated in the high corona as "beams" (the collisional thick-target model; Brown 1971; Emslie 1978), but recently it has been suggested that limits on electron numbers and propagation effects (e.g., beam instabilities) require an alternative mode of energy transport, such as acceleration of particles in the lower corona or chromosphere by Alfvén waves (Fletcher \& Hudson 2008). Continuum measurements from spectra are necessary to test heating models by providing constraints on the optical depths and electron densities that are attained in the deepest layers of the flare atmosphere.

Spatially resolved flare spectra have been obtained at NUV/blue wavelengths from the ground and have shown a range of spectral properties around the expected location of the Balmer jump (Neidig 1983; Kowalski et al. 2015a). The continuum emission from these spectra has been interpreted as optically thin hydrogen recombination radiation (Neidig \& Wiborg 1984), although an emission component from increased $\mathrm{H}^{-}$emission in the upper photosphere has also been suggested to explain some of the observed variation (Hiei 1982; Boyer et al. 1985). However, the brightest regions of solar flares were rarely and poorly sampled in the past: the locations of the spectrographic slit relative to the small whitelight footpoints were not precisely known (Neidig \& Wiborg 1984; Neidig 1989), due to the rapid spatial and temporal evolution of the emission and also to variable and poor seeing during flares observed from the ground prior to the advent of adaptive optics (Donati-Falchi et al. 1984). Therefore, the most extreme atmospheric conditions in the lower atmosphere during solar flares are not well constrained.

Recently, Sun-as-a-star observations from $\mathrm{SOHO} / \mathrm{VIRGO}$ 's Sun PhotoMeter (SPM) have indicated the presence of a hotter blackbody emission component in the optical with a color temperature of $T \sim 9000 \mathrm{~K}$ (Kretzschmar 2011) which implies very large heating at high densities. High spatial resolution observations from Hinode during two X-class flares have shown a much lower color temperature in the optical of only $T \sim 5000-6000 \mathrm{~K}$ (Watanabe et al. 2013; Kerr \& Fletcher 2014), which is consistent with photospheric heating by several hundred $\mathrm{K}$ or optically thin hydrogen recombination radiation from heating of the mid-chromosphere. Blackbody fitting of NUV and optical spectra and broadband photometry of magnetically active $M$ dwarf stars in the gradual and impulsive phases of flares results in larger color temperatures, $T \gtrsim 8000 \mathrm{~K}$ but typically $T \sim 9000-12,000 \mathrm{~K}$ (Hawley \& Fisher 1992; Hawley et al. 2003; Zhilyaev et al. 2007; Fuhrmeister 
et al. 2008; Kowalski et al. 2010, 2013). Clearly, a more thorough spatially resolved characterization of the brightest flare footpoints is necessary to determine the prevalence of hot blackbody-like emission in solar flares, and if any proposed heating model can self-consistently explain the implied heating requirements.

Previous spectral observations of the NUV and optical have been interpreted using static isothermal slab models (e.g., Donati-Falchi et al. 1985) or semi-empirical static models (Machado et al. 1980; Mauas et al. 1990; Kleint et al. 2016), but the flare atmosphere is known to be highly dynamic and stratified (Cauzzi et al. 1996; Falchi \& Mauas 2002). It has been proposed that the continuum emission may originate in impulsively generated downflows in the upper chromosphere (Livshits et al. 1981; Gan et al. 1992), or chromospheric "condensations" (hereafter CC; Fisher et al. 1985; Fisher 1989), which are also attributed to the formation of $\mathrm{H} \alpha$ red-wing emission components that are often observed in solar flares (Ichimoto \& Kurokawa 1984; Canfield \& Gayley 1987; Canfield et al. 1990). Kowalski et al. (2015b) recently found that an extremely large electron beam flux of $10^{13} \mathrm{erg} \mathrm{cm}^{-2} \mathrm{~s}^{-1}$ could produce hot $T \sim 10,000 \mathrm{~K}$ blackbody-like emission in very dense CCs. With current computational facilities and constraints on electron beam fluxes from the Reuven Ramaty High Energy Solar Spectroscopic Imager (RHESSI; Lin et al. 2002) and high spatial resolution imagery, it is timely to critically examine the hydrodynamic and time-dependent radiative response of the models and compare to new spectral observations of solar flares.

We have begun a large campaign to characterize the emission properties of the brightest flaring magnetic footpoints during Cycle 24 flares using new NUV and far-ultraviolet (FUV) spectra from the Interface Region Imaging Spectrograph (IRIS; De Pontieu et al. 2014). The high spatial resolution of IRIS allows improved intensity measurements of the continuum emission, which is observed in compact sources, or kernels, as small as 0!'3 (Jess et al. 2008). In this paper, we present radiative-hydrodynamic (RHD) modeling of the brightest continuum flaring pixels in the X1 flare of 2014 March 29, which has been extensively studied by Judge et al. (2014), Young et al. (2015), Liu et al. (2015), Battaglia et al. (2015), Kleint et al. (2015), Matthews et al. (2015), Kleint et al. (2016) and Rubio da Costa et al. (2016). In Heinzel \& Kleint (2014) and Kleint et al. (2016), the bright NUV continuum emission from this flare was identified and compared to static beam heated model atmospheres from Ricchiazzi \& Canfield (1983) and to static phenomenological models with the RH code. Heinzel \& Kleint (2014) concluded that the NUV continuum intensity was consistent with optically thin Balmer continuum emission. However, the time-dependent radiative transfer and the hydrodynamics, which can affect beam propagation through evaporation and condensation, have not yet been compared in detail to the continuum observations; Heinzel et al. (2016) and Rubio da Costa et al. (2016) present new RHD simulations with the Flarix and RADYN codes, respectively, for relatively low beam fluxes compared to the flux inferred from imaging spectroscopy of the brightest source in the flare (Kleint et al. 2016). In this paper, we use the state-of-the-art Fokker-Planck treatment of energy deposition (Mauas \& Gomez 1997; Liu et al. 2009; Battaglia et al. 2012; Allred et al. 2015) from a high-flux electron beam, in order to understand the time evolution of the atmospheric stratification that self-consistently explains both the NUV continuum emission and chromospheric line profiles in the brightest flaring footpoints.

By rigorously testing new RHD models guided by the combined information from RHESSI and new data of the whitelight continuum and chromospheric lines, we seek answers to the following questions:

1. Using electron beam parameters inferred from standard thick target modeling of RHESSI X-ray data, do electron beams produce an atmospheric and radiative response that is consistent with the high spatial and spectral line and continuum constraints from IRIS?

2. Does a CC form that is hot and dense enough to explain the observed IRIS line and continuum emission?

3. Does the hydrodynamic response of the atmosphere to beam heating result in flare continuum emission in the IRIS NUV channel that is predominantly optically thin hydrogen recombination radiation? Is there evidence for hot $(T \gtrsim 9000 \mathrm{~K})$ blackbody-like radiation from photospheric densities?

The paper is organized as follows. In Sections 2 and 3, we describe the calibration of the IRIS observations and the high spatial resolution flare footpoint development; in Section 4, we discuss the constraints on the continuum intensity and emission line profiles in the spectra of the brightest flaring footpoints; in Section 5 we describe the radiative-hydrodynamic modeling of these spectra and the formation of the NUV continuum and Fe II emission lines; in Section 6, we compare the modeling results to the brightest sources in the slit jaw images; in Section 7 we discuss several limitations of the modeling and future work; in Sections 8 and 9 we present our conclusions. Appendices A and B discuss broader wavelength model predictions for the optical continuum emission.

\section{Intensity Calibration of the IRIS Observations}

IRIS is a rastering spectrograph with simultaneous coverage in the near-ultraviolet (NUV, 2782.7-2835.1 $\AA$ ) and far-ultraviolet (FUV1, 1331.7-1358.4 A; FUV2, 1389.0-1407.0 Å) including both lines and continua. IRIS has a spatial resolution of 0 "! 33 in the FUV and 0". 4 in the NUV. The IRIS observations covering the GOES X1 flare SOL2014-03-29T17:48 were obtained with an 8-step raster across AR 12017 (NOAA 12017, $\mu=0.82$ ). We discuss the flare footpoint development of this flare in Section 3.2

The 8-step raster was obtained with an $8 \mathrm{~s}$ exposure time per step, resulting in a $75 \mathrm{~s}$ raster cycle. During the flare, the automatic exposure control (AEC) decreased the NUV spectral exposure times to $2.4 \mathrm{~s}$ at UT 17:46:13. The spectra were binned in the dispersion direction in the FUV only, resulting in a spectral pixel width of $\sim 26 \mathrm{m \AA}$ in both channels. We employ the $2830 \AA$ slit-jaw (SJI 2832) images which remained unsaturated for the duration of the flare and have the same spatial scale $\left(0\right.$. ! 166 pixel $\left.^{-1}\right)$ as the spectra. The SJI 2832 images (\#0 to \#179) are obtained once per raster at the third slit position. The bandpass of SJI 2832 has a FWHM of $4 \AA$ centered on $\lambda=2830 \AA$, which is far into the red absorption wing of $\mathrm{Mg}$ II $h$.

IRIS level 3 data cubes were created by shifting the NUV spectra by -2 pixels and the FUV spectra by +1 pixels in order to align with the fiducial marks of SJI 2832. The NUV wavelength calibration was adjusted by $+0.025 \AA$ based on the alignment of the Ni I 2799.474 A line. We applied an intensity 
calibration to the spectra (IRIS Technical Note 24; Kleint et al. 2016) and SJI 2832 using the post-flight effective area curves $\left(A_{\text {eff }}(\lambda)\right)$ from the routine iris_get_response.pro developed by J. P. Wuesler. Specifically, the conversion of the spectra with calibrated units of DN s${ }^{-1}$ pixel $^{-1}$ to units of erg $\mathrm{cm}^{-2} \mathrm{~s}^{-1}$ s.r. $^{-1} \AA^{-1}$ was performed by multiplying by the following factor:

$$
C=\frac{\text { gain }}{A_{\text {eff }}(\lambda) \Delta \lambda} \times S \times E,
$$

where the gain is 18 photons $\mathrm{DN}^{-1}$ for the NUV spectra, 4 photons $\mathrm{DN}^{-1}$ for the FUV spectra, $\Delta \lambda$ is the dispersion, $S$ is the solid angle conversion from pixel ${ }^{-1}$ to steradian ${ }^{-1}$ for a slit width of $0 . " 33$, and $E$ is the energy per photon. We convert the SJI 2832 calibrated count rate (DN s${ }^{-1}$ pixel $^{-1}$ ) to an equivalent (constant) continuum intensity over the SJI bandpass by using Equation (1) with $A_{\text {eff }}(\lambda) \Delta \lambda$ replaced by $\int A_{\text {eff }}(\lambda) d \lambda=0.02 \mathrm{~cm}^{2} \AA$ as a proxy for the line and continuum emission brightness in the SJI 2832 images during the flare.

\section{IRIS Slit Jaw 2832 Image Analysis}

Compact flaring magnetic footpoints are readily identified using the high spatial resolution SJI 2832 data. The flare intensity in SJI 2832 is also used to calculate a flare footpoint area, which we compare to the area obtained from partially unresolved RHESSI hard X-ray observations as described in Kleint et al. (2016). The flare footpoint area is critical for inferring a nonthermal electron beam energy flux, which is an input for 1D RHD modeling (Section 5).

We calculate the excess intensity in SJI 2832 at each time during the flare by subtracting a pre-flare image (SJI 2832 \#171). Because the observing cadence (75 s) is relatively low compared to the timescale $(\sim 120 \mathrm{~s})$ of the hard X-ray impulsive phase of the flare, we use a pre-flare image instead of a running difference to define the flare area. It should be kept in mind that the flare area consists of both newly heated flare regions and decaying flare regions that were previously heated, and we do not know if the bright pixels in this flare are observed during their rising or decaying evolution.

In Figure 1 (top panel) we show the SJI 2832 \#173 image which exhibits brightness variations outside the flare ribbons due to the sunspots and granulation. As a result of the evolution of granulation and a drift in the instrument pointing, temporal variations of $10 \%-20 \%$ are present in the excess intensity images. Thus we define the flare area using two significance thresholds as follows:

1. Low Threshold Excess ("low thresh"). We subtract a preflare image (\#171) from each image in the observation. For the excess images prior to \#171, we find that the standard deviation of the excess count rate approaches a value of $\approx 15 \mathrm{DN} \mathrm{s}^{-1}$ pixel $^{-1}$ before the flare, which is due to the granulation variation. Our low threshold excess area ("low thresh") corresponds to the flare area with an excess count rate of $60 \mathrm{DN} \mathrm{s}^{-1}$ pixel $^{-1}$, which is $\approx 0.6 \times 10^{6} \mathrm{erg} \mathrm{cm}^{-2} \mathrm{~s}^{-1}$ s.r. $^{-1} \AA^{-1}$ or $4 \sigma$.

2. High Threshold Excess ("high thresh"). We seek to determine the physical processes that produce the continuum intensity in the brightest footpoints in the flare determined by the SJI 2832 sources. We define a high threshold excess that corresponds to an excess count
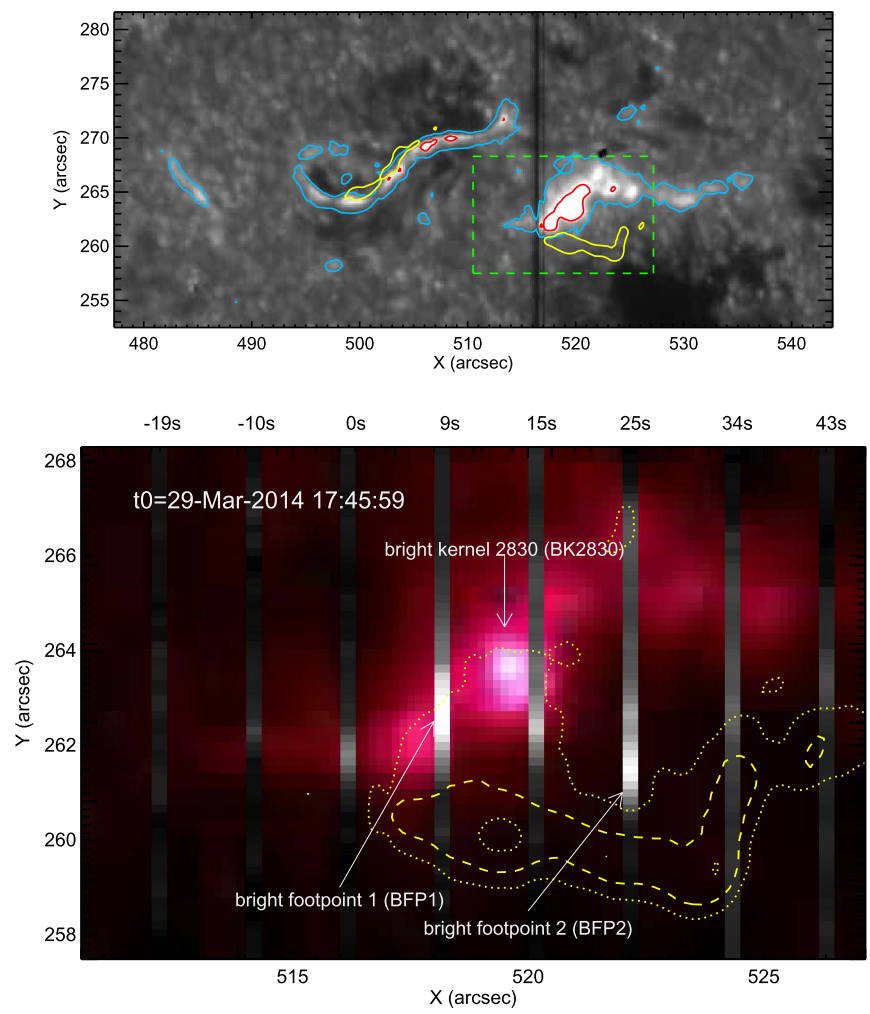

Figure 1. (Top) Total intensity in SJI 2832 \#173. The areas corresponding to the high thresh (red) and low thresh (light blue) values for the excess intensity SJI 2832 \#173 at the time of 2014 March 29 17:45:59 are overlayed. The high thresh area for SJI 2832 \#174 is shown in yellow contours. (Bottom) An expanded view of the green dashed box in the top panel, showing the excess intensity of SJI 2832 \#173. The spectroscopic raster \#173 during the hard $\mathrm{X}$-ray impulsive phase is shown for the excess C2826 (NUV continuum) in gray scale, and the times for each spectrum relative to $t 0$ are indicated on the top $x$-axis. The high thresh $\left(3 \times 10^{6} \mathrm{erg} \mathrm{cm}^{-2} \mathrm{~s}^{-1}\right.$ s.r. $\left.{ }^{-1} \AA^{-1}\right)$ area for SJI $2832 \# 174$ is indicated by a yellow dashed contour. The yellow dotted contours show the area with excess intensity above $1.5 \times 10^{6} \mathrm{erg} \mathrm{cm}^{-2} \mathrm{~s}^{-1}$ s.r. ${ }^{-1} \AA^{-1}$ and $6 \times 10^{6} \mathrm{erg} \mathrm{cm}^{-2} \mathrm{~s}^{-1}$ s.r. $^{-1} \AA^{-1}$ (half of high thresh value and twice the high thresh value, respectively). BK2830 is located at $(x, y)=(519.5,263.8)$. The ribbon progresses downward over time and the IRIS slit crossed two locations of bright NUV flare continuum emission as indicated by BFP1 and BFP2 with arrows.

rate of $\sim 290 \mathrm{DN} \mathrm{s}^{-1} \mathrm{bin}^{-1}$ or $3 \times 10^{6} \mathrm{erg} \mathrm{cm}^{-2} \mathrm{~s}^{-1}$ s.r. ${ }^{-1} \AA^{-1}$, which is a factor of five larger than the lowthresh value. The high-thresh level corresponds to $\sim 30 \%$ of the maximum excess pixel value in SJI 2832 \#173. The low- and high-thresh areas are shown as contours on the total intensity image SJI 2832 \#173 in Figure 1 (top panel).

An approximate comparison between temporally contiguous and spatially adjacent measurements of the continuum intensity from the spectra and in SJI 2832 justifies the high-thresh value. The spectral region at $\lambda=2825.6-2825.9 \AA$ (hereafter, C2826) shows bonafide continuum emission (Section 4). At the same spatial (y) location in the excess spectra and in the excess SJI 2832 image, the spatial extent in the $y$-direction of the excess spectral continuum $(x=518$ ". 2 at the raster position corresponding to $t 0+9 \mathrm{~s}$ in Figure 1 (bottom)) has a FWHM of $\approx 11$ pixels, or 1 !" 8 with a half-maximum excess intensity value of $1.1 \times 10^{6} \mathrm{erg} \mathrm{cm}^{-2} \mathrm{~s}^{-1}$ s.r. ${ }^{-1} \AA^{-1}$. At one raster step earlier in SJI $2832 \# 173$ 


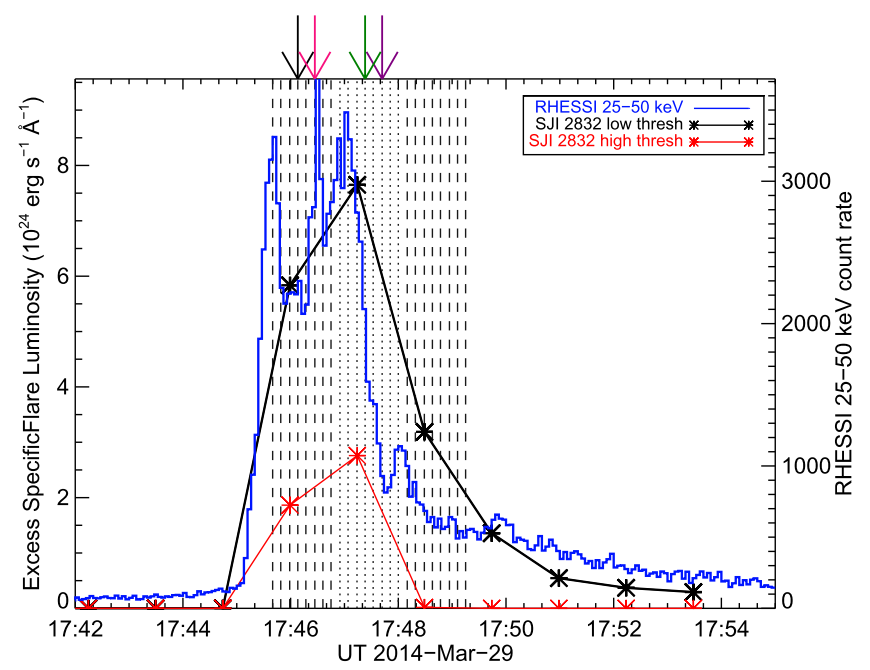

Figure 2. Excess specific flare luminosity in the SJI 2832 integrated over the low thresh and high thresh areas (left axis) compared to the corrected count rate RHESSI hard X-ray $E=25-50 \mathrm{keV}$ light curve (summed for the detectors $1 \mathrm{~F}$, $2 \mathrm{~F}, 3 \mathrm{~F}, 4 \mathrm{~F}, 5 \mathrm{~F}, 6 \mathrm{~F}, 7 \mathrm{~F}$, and $9 \mathrm{~F}$; right axis). The vertical dashed and dotted lines indicate the times of the spectral raster observations (the leftmost grouping of vertical dashed lines corresponds to raster $\# 173$, the vertical dotted lines to raster \#174). The arrows at the top indicate the times of the four flare spectra shown in Figure 3, and are color-coded to the spectra. The spectral observations of bright NUV continuum emission in BFP1 at 17:46:08 (black arrow) and BFP2 at 17:46:24 (pink arrow) correspond to the middle of the impulsive phase of the hard X-ray light curve.

(corresponding to $t 0$ in Figure 1), we find that a N-S spatial extent of 11 pixels (thus, the same extent as the FWHM of the C2826 continuum emitting area in the spectra) corresponds to an excess slit jaw intensity of approximately $3 \times 10^{6} \mathrm{erg} \mathrm{cm}^{-2} \mathrm{~s}^{-1}$ s.r. $^{-1} \AA^{-1}$. Thus, a high-thresh value of $3 \times 10^{6} \mathrm{erg} \mathrm{cm}^{-2} \mathrm{~s}^{-1}$ s.r. $^{-1} \AA^{-1}$ from SJI 2832 reasonably represents an area corresponding to bright continuum emission, with an excess C2826 value $I_{\lambda, \text { excess }}>10^{6} \mathrm{erg} \mathrm{cm}^{-2} \mathrm{~s}^{-1}$ s.r. $^{-1} \AA^{-1}$ (assuming that the emission has not significantly decayed over the time from slit jaw image to the spectral observation). The high-thresh value is $70 \%$ of the maximum over the $y$-direction profile at the time of SJI $2832 \# 173$, and suggests that excess flare continuum intensity constitutes $\approx 50 \% \quad\left(2.2 \times 10^{6} \mathrm{erg} \mathrm{cm}^{-2} \mathrm{~s}^{-1}\right.$ s.r. $^{-1} \AA^{-1} /$ $4.3 \times 10^{6} \mathrm{erg} \mathrm{cm}^{-2} \mathrm{~s}^{-1}$ s.r. $^{-1} \AA^{-1}$ ) of the excess intensity in the SJI 2832 images.

\subsection{Comparison to Hard X-Ray Emission}

RHESSI data provide critical information on the flare impulsive-phase hard X-ray emission, which is used to infer a single or double power-law distribution of an electron beam using the collisional thick-target model (e.g., Holman et al. 2003; Milligan et al. 2014). The collisional thick-target modeling of the hard X-ray RHESSI data and the hard X-ray imaging for this flare has been performed by Battaglia et al. (2015), Kleint et al. (2016), and Rubio da Costa et al. (2016), and we use representative parameters from these fits for RHD modeling (Section 5) of the early impulsive phase before the bright thermal component becomes very bright in the X-ray spectrum.

The hard X-ray $E=25-50 \mathrm{keV}$ RHESSI light curve (after applying the approximate adjustment for attenuator state changes) is shown in Figure 2 with the times of the IRIS spectral observations are indicated by vertical dashed and dotted lines. The $E=25-50 \mathrm{keV}$ light curve defines the impulsive phase of the flare (120 s FWHM), and consists of several peaks with varying durations of 8-30s superimposed on a gradually varying emission component. The eight slit positions in Figure 1 (bottom) correspond to raster \#173, and the times of these spectral observations are indicated by the leftmost eight vertical dashed lines in Figure 2. We refer to SJI 2832 \#173 (17:45:59) as the "mid peak phase" and SJI 2832 \#174 (17:47:14) as the "early fast decay phase" according to the phase of the hard X-ray light curve. Notably, SJI 2832 \#173 occurs just after the hard X-ray event peaking between $17: 45: 36-17: 45: 48$ that is evident in the high energy bands $E>25 \mathrm{keV}$ in Figure 2 .

The excess specific luminosity from the low- and high-thresh flare areas in SJI 2832are shown in Figure 2; the time profiles are generally similar to the coarse evolution of the $E=25-50 \mathrm{keV}$ X-ray light curve. The areal evolution of the low-thresh (not shown) is similar to the specific luminosity but exhibits a faster decay relative to the peak. During the impulsive phase (SJI 2832 \#173 and 174), the low thresh area is approximately a factor of 10 larger than the high thresh area, but only $30 \%$ of the excess specific flare luminosity originates from the high-thresh area. In the gradual phase of the hard X-ray light curve, the high thresh area is just six pixels, and thus this threshold effectively separates the impulsive and gradual phases of the hard X-ray emission in this flare.

\subsection{Flare Footpoint Evolution in SJI 2832}

In Figure 1 (top panel), we show the spatial evolution in SJI 2832 of the low-thresh and high-thresh flare areas. The highthresh areas are shown as red (SJI $2832 \# 173$ ) and yellow (SJI 2832 \#174) contours, and the low-thresh (SJI 2832 \#173) are the light blue contours. Similar to recent high spatial resolution data of other C, M, and X-class two-ribbon flares (Krucker et al. 2011; Sharykin \& Kosovichev 2014; Kowalski et al. 2015a), one relatively narrow (NE) ribbon develops across the umbra and another (SW) ribbon that is more spatially diffuse develops in the plage. From the mid peak phase (SJI $2832 \# 173$ ) to early fast decay phase (SJI $2832 \# 174$ ), the location of the high thresh flare area changes relatively rapidly, following motions that are both perpendicular and parallel to the magnetic polarity inversion line, which runs diagonally from SE to NW through the spots at $\left(508^{\prime \prime}, 270^{\prime \prime}\right)$ (Kleint et al. 2015). From the mid peak to the early fast decay phases, the (apparent) motion of the high-thresh footpoints in the SW (plage) ribbon is predominantly perpendicular to the polarity inversion line (nearly parallel to the slit in the image $y$ direction), as can be seen by comparing the high-thresh locations at the two times in Figure 1. However, the brightest regions of the $\mathrm{NE}$ (umbral) ribbon rapidly move from the western to the eastern side of the ribbon (in the direction away from the IRIS slit). The SW ribbon moves rapidly through the plage toward the large sunspot of negative polarity (Kleint et al. 2015) at $\left(530^{\prime \prime}, 255^{\prime \prime}\right)$; the average apparent speed of this ribbon in the image $y$-direction is $30-40 \mathrm{~km} \mathrm{~s}^{-1}$ and is as high as $60 \mathrm{~km} \mathrm{~s}^{-1}$ at some locations. The NE ribbon moves apparently slower in the direction perpendicular to the polarity inversion line, since it develops over the umbrae of the spots where its spatial development may be limited by the umbral magnetic field. 

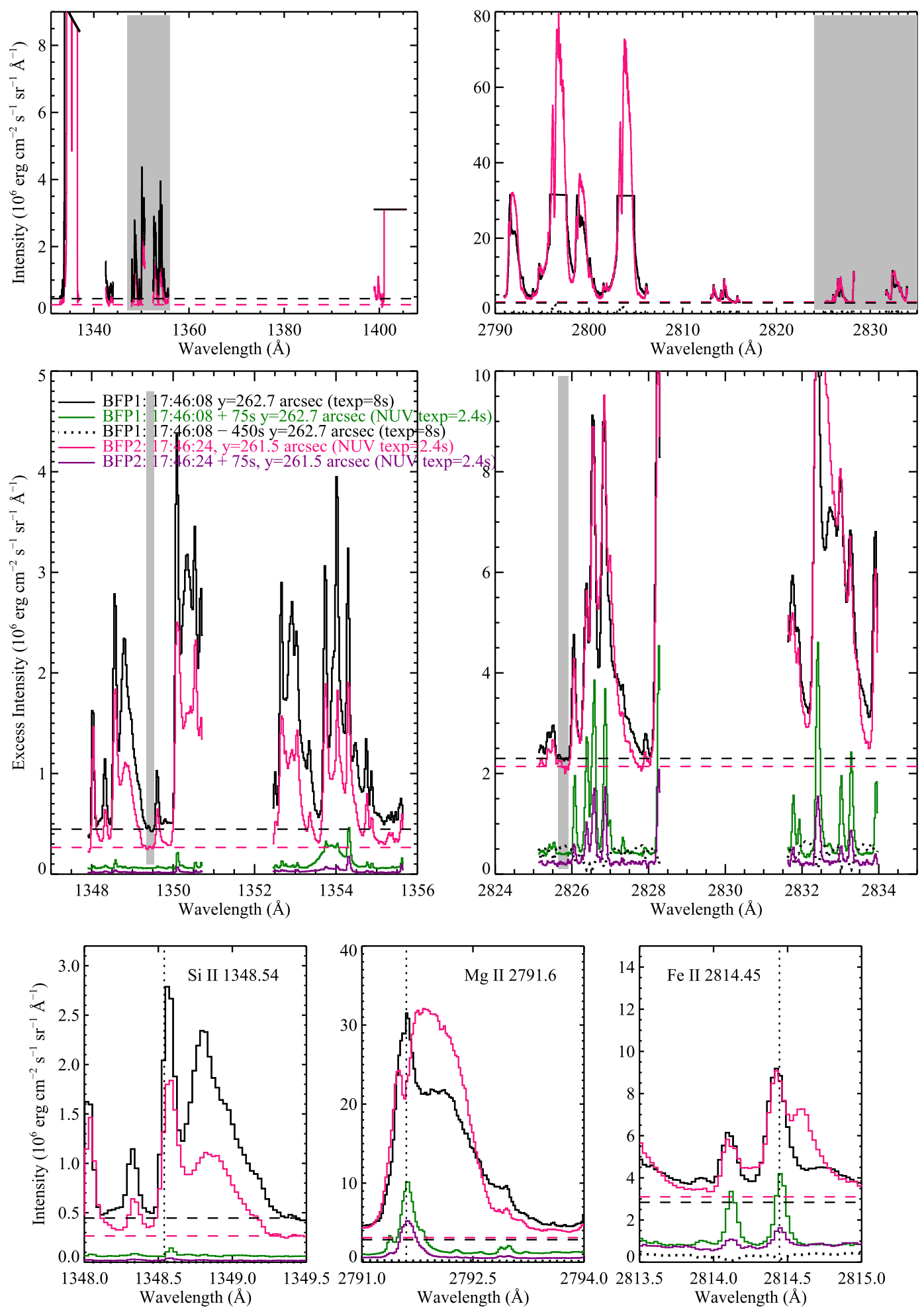

Figure 3. (Top) FUV and NUV spectra extracted from the brightest flaring pixels in Figure 1 at $t 0+9 \mathrm{~s}$ (BFP1, black) and at $t 0+25 \mathrm{~s}$ (BFP2, pink) over the wavelength range of IRIS. Note that only select wavelength regions of the full possible spectral coverage were recorded for the observations of this flare. (Middle) Enlarged view of the wavelength ranges indicated by the shaded gray regions in the top panel. The wavelength ranges of the continuum regions C1349 and C2826 are indicated by shaded vertical bars. The spectra at the same spatial positions as BFP1 and BFP2 are shown one raster later (\#174) as dark green and purple spectra, respectively. A pre-flare spectrum is shown for the NUV as a dotted spectrum. (Bottom) Selected chromospheric spectral lines for the same locations and times as the spectra in the middle panels; note that the pre-flare spectrum has not been subtracted here. Each singly ionized species shows a redshifted emission component and a component centered near the rest wavelength. The redshifted emission component is much less prominent as the continuum level decreases in the hard X-ray fast decay phase. In all panels, the horizontal dashed lines are the values of C1349 and C2826 from BFP1 at 17:46:08 and BFP2 at 17:46:24 extrapolated to all wavelengths. 
In Figure 1 (bottom panel) we show a zoomed region of the excess intensity in the SW (plage) ribbon at SJI 2832 \#173 $(t 0)$. This ribbon contains the brightest kernel (BK2830) in the flare at $(x, y)=(519$ !' 5,263 !' 8$)$. The high-thresh contours of the excess intensity are shown for SJI 2832 image \#174, which illustrates that a trail of emission extends from BK2830 in SJI $2832 \# 173$ to the brightest regions in the ribbon in SJI 2832 \#174 as the ribbon has apparently moved toward the SW umbra. The spectroscopic slit positions and relative timing are indicated, showing that the slit does not cross the brightest regions of BK2830; the brightest points in BK2830 move diagonally from NW to SE as the raster "hops" over it from $t 0+9 \mathrm{~s}$ to $t 0+15 \mathrm{~s}$. Imaging spectroscopy of RHESSI data shows that the brightest hard X-ray source corresponds approximately to this location (Battaglia et al. 2015; Kleint et al. 2016). After SJI 2832 \#174 (in the hard X-ray gradual phase), the ribbons become fainter and they continue to separate but at significantly slower apparent speed.

\section{Spectral Analysis of the Brightest Footpoint Emission}

In this section we calculate the continuum intensity and chromospheric line bisector velocities for the two brightest NUV pixels, labeled "bright footpoint 1" and "bright footpoint 2 " in Figure 1 (bottom). The quantities from these spectra will be compared to the RHD model outputs in Section 5 .

\subsection{Continuum Intensity}

The continuum intensity at the brightest footpoints provides rigorous constraints for flare heating model predictions. We search all spectra for bright $\mathrm{Mg}$ II $\lambda 2791.6$ and excess C2826 emission to identify the brightest flare sources that cross the IRIS slit. We calculate the excess continuum intensity in the FUV (1349.35-1349.53 $\AA$; hereafter C1349) and in the NUV (2825.64-2825.90 ^; C2826). The excess values are obtained by subracting the pre-flare continuum intensity at the same spatial location. For the emission line analysis, the pre-flare is not subtracted because this can affect the line profiles. ${ }^{7}$ The excess C2826 raster image in Figure 1 (bottom) shows the two brightest flaring locations in $\mathrm{Mg}$ II and in the $\mathrm{C} 2826$ continuum, which are labeled "bright footpoint \#1" (hereafter, BFP1) at the 4th raster position and "bright footpoint \#2" (hereafter, BFP2) at the 6th raster position in raster \#173. Most of the bright regions in the excess $\mathrm{C} 2826$ raster image aligns with the excess intensity in the SJI 2832 images, but the location of BFP2 in the 6th raster position appears between the high-thresh contours of SJI 2832 \#173 and \#174; this can be attributed to the rapid spatial development of the flare ribbon toward the SW umbra over the time of the spectral raster (Figure 1). The spectra from BFP1 (black) and BFP2 (pink) are shown in the top panel of Figure 3. The BFP1 spectra (black) were obtained with exposure times of $8 \mathrm{~s}$ in the FUV and NUV, and the BFP2 spectra (pink) were obtained with an exposure time of $8 \mathrm{~s}$ in the FUV and $2.4 \mathrm{~s}$ in the NUV. In the FUV, the major emission lines (e.g., C II and Si IV) are saturated for both spectra, but the $\mathrm{Mg}$ II $\mathrm{h}+\mathrm{k}$ lines in the NUV are not saturated for the spectrum of BFP2. The Mg II triplet $\lambda 2791.6$ is not saturated in either spectra.

\footnotetext{
The continuum shape can also be affected by subtracting the pre-flare (Kleint et al. 2016). Whereas a physical quantity (velocity) is inferred from the line bisector, the observed excess is used only as a direct comparison to the model excess.
}

The excess NUV and FUV spectra for BFP1 (black) and BFP2 (pink) are shown in the middle panel of Figure 3 for the wavelength regions indicated by gray bars in the top panel of Figure 3. The wavelength regions of C1349 and C2826 are shown as gray bars in the middle panel of Figure 3. These wavelength regions are continuum regions outside of major and minor flare emission lines (the NUV continuum wavelength range is the region considered by Heinzel \& Kleint 2014; Kleint et al. 2016). We note that although the gray bar in Figure 3 corresponding to $\mathrm{C} 1349$ does not include any prominent emission lines, this wavelength region includes the rest wavelength of Fe XII $\lambda 1349.4$. This wavelength region is nonetheless the most optimal estimate of the continuum among the limited wavelength regions that were read out for these observations. The times of BFP1 and BFP2 compared to the RHESSI impulsive phase are indicated by the two leftmost arrows at the top of Figure 2. BFP1 occurs after the hard X-ray peak from $17: 45: 36$ to $17: 45: 48$, and there is a short hard X-ray event near the time of BFP2, indicated by the pink arrow in Figure 2. Extrapolations of C2826 and C1349 over the NUV and FUV spectral ranges are shown as horizontal dashed lines, which indicate that these continua are the lower pedestal of the emission compared to the rest of the spectra. A preflare spectrum and a spectrum corresponding to the early fast decay phase (raster \#174) at the locations of BFP1 and BFP2 are also shown in Figure 3 to illustrate the evolution of the excess continuum emission. Within one raster (75 s), the excess NUV continuum values have decreased by a factor of $>4$, which is consistent with the rapid motion of the ribbon front toward the sunspot (Figure 1).

The times, locations, and measured intensity values of $\mathrm{C} 2826$ and $\mathrm{C} 1349$ are given for BFP1 and BFP2 in Table 1. The values of the excess continuum intensity of BFP1 and BFP2 are $\sim 2.1-2.2 \times 10^{6} \mathrm{erg} \mathrm{cm}^{-2} \mathrm{~s}^{-1}$ s.r. $^{-1} \AA^{-1}$ and $\sim 0.27-0.45 \times$ $10^{6} \mathrm{erg} \mathrm{cm}^{-2} \mathrm{~s}^{-1}$ s.r. $^{-1} \mathrm{~A}^{-1}$ for the $\mathrm{C} 2826$ and $\mathrm{C} 1349$ continuum regions, respectively. The ratio of excess NUV to excess FUV continuum intensity for BFP1 is $\sim 5$. For BFP2, the exposure times are not the same for the NUV and FUV due to the IRIS AEC adjustment. The intensity contrast in the NUV for BFP1 and BFP2 are $200 \%-400 \%$ (Kleint et al. 2016), which is a large range due to a significant spatial variation in the NUV continuum in the preflare spectrum (which typically varies from pixel to pixel by $10^{5} \mathrm{erg} \mathrm{cm}^{-2} \mathrm{~s}^{-1}$ s.r. $^{-1} \AA^{-1}$ or $10 \%-20 \%$ ). A third bright footpoint (BFP3) in the 5th raster position at $(x, y) \sim\left(520^{\prime \prime}, 262^{\prime \prime}\right)$ in Figure 1 exhibits an excess C2826 of $\sim 1.5 \times 10^{6} \mathrm{erg} \mathrm{cm}^{-2} \mathrm{~s}^{-1}$ s.r. ${ }^{-1} \AA^{-1}$; but this footpoint is not analyzed further in this paper.

\subsection{Bisector Velocity of the Asymmetric Chromospheric Line Profiles}

In Figure 3 (bottom panels), we show the emission line profiles of $\mathrm{Si}$ II $\lambda 1348.54$, the $\mathrm{Mg}$ II triplet $\lambda 2791.6$, and Fe II $\lambda 2814.45$ for BFP1 and BFP2. The rest wavelengths are indicated as vertical dotted lines. A redshifted, broadened emission component is present in each of these line profiles, as discussed by Liu et al. (2015) for this flare for the $\mathrm{Mg}$ II $h+k$ lines. We refer to this redshifted emission component as a "red wing asymmetry" (RWA), since this redshifted component is qualitatively similar to the red wing asymmetries observed in $\mathrm{H} \alpha$ profiles from Ichimoto \& Kurokawa (1984). A spectrally resolved RWA component is no longer present by the next raster $75 \mathrm{~s}$ later (dark green and purple spectra), while the emission centered near the rest wavelength remains relatively 
Table 1

IRIS Continuum Observations

\begin{tabular}{lcccccc}
\hline \hline label & mid-time & raster \# & $x\left[^{\prime \prime}\right]$ (raster position) & $y\left[^{\prime \prime}\right]$ (pixel) & $\begin{array}{c}\mathrm{C} 2826 \text { (pre) } \\
{\left[10^{6} \mathrm{erg} \mathrm{cm}^{-2} \mathrm{~s}^{-1} \mathrm{s.r}^{-1} \AA^{-1}\right]}\end{array}$ & $\begin{array}{c}\mathrm{C} 1349 \text { (pre) } \\
{\left[10^{6} \mathrm{erg} \mathrm{cm}^{-2} \mathrm{~s}^{-1} \mathrm{s.r}^{-1} \AA^{-1}\right]}\end{array}$ \\
\hline BFP1 & $17: 46: 08$ & 173 & $518.2(4)$ & $262.7(437)$ & $2.84(0.54)$ & $0.45(\ldots)$ \\
BFP2 & $17: 46: 24$ & 173 & $522.2(6)$ & $261.5(430)$ & $3.10(0.96)$ & $0.27(\ldots)$ \\
\hline
\end{tabular}

Note. The $y$-pixel corresponds to the $y$-pixel location after a +1 pixel and -2 pixel shift has been applied to obtain the level 3 FUV and NUV spectral datacubes, respectively. For the $x$ and $y$ positions, IDL indices of level 3 datacubes correspond to the values in the parentheses after subtracting 1 .

bright. We interpret the RWA as evidence of downflowing, heated chromospheric material as discussed for the $\mathrm{Mg}$ II lines in Graham \& Cauzzi (2015) for the X-class flare SOL2014-0910T17:45. The rapid disappearance of the RWA component over the raster cycle of $75 \mathrm{~s}$ is consistent with the findings of Graham \& Cauzzi (2015) showing that the condensation lifetime is $\sim 30-60 \mathrm{~s}$.

Following Graham \& Cauzzi (2015), we measure the $30 \%$ bisector of the $\mathrm{Mg}$ II triplet line to infer a chromospheric velocity. ${ }^{8}$ The bisector velocities for the BFP1 and BFP2 spectra in Figure 3 are $\sim 40 \mathrm{~km} \mathrm{~s}^{-1}$, but the detailed line profiles differ considerably between the two spectra.

\subsubsection{NUV Fe II Emission Line Analysis}

Many Fe II emission lines become bright in the NUV during the flare and exhibit complex line profiles that can be used as a diagnostic of the flare chromosphere (Walkowicz et al. 2008). These lines are never saturated in IRIS flare spectra and are thus a useful parameter to compare among flares. Moreover, they are much more optically thin than other, brighter chromospheric flare lines such as the Mg II lines and may provide a favorable alternative as a diagnostic of the flare velocity field through the white-light continuum emitting layers. The Fe II lines have not yet been characterized in IRIS flare spectra. In this section we describe the properties of the line profiles and in Section 5.6 we compare to model predictions.

The Fe II flare lines in the NUV typically have upper levels with excitation energies of $E / h c \sim 61,000 \mathrm{~cm}^{-1}$, and we focus our analysis on the Fe II $\lambda 2814.45$ and Fe II $\lambda 2832.39$ lines. The line profiles for BFP1 and BFP2 are shown in the top panels of Figures 4 and 5 for Fe II $\lambda 2814.45$ and Fe II $\lambda 2832.39$, respectively. The Fe II $\lambda 2814.45$ and Fe II $\lambda 2832.39$ lines exhibit an emission component with a peak that is within 1 pixel of the rest wavelength and a RWA emission component that peaks at least 5 pixels to the red of the of the rest wavelength.

The Fe II $\lambda 2814.45$ line is one of the least blended with other emission lines, thus allowing a clean characterization of the RWA emission component. The peaks of the RWA emission components for this line in BFP1 and BFP2 are indicated by "RWA" in Figure 4. In the BFP1 spectrum (black) of Fe II $\lambda 2814.45$, the RWA component is much less intense than the peak of the line at rest-wavelength (hereafter, indicated as $\lambda_{\text {rest }}$ ) and is shifted to the red to $\lambda \sim 2814.75 \AA$. The peaks of the RWA components of Fe II $\lambda 2814.45$ are redshifted to $\lambda$ $-\lambda_{\text {rest }}=16 \mathrm{~km} \mathrm{~s}^{-1}$ (BFP2) and $\lambda-\lambda_{\text {rest }}=32 \mathrm{~km} \mathrm{~s}^{-1}$ (BFP1), which are indicated by vertical lines with $\lambda_{\text {rest }}$ in Figure 4. Notably, the emission line components at $\lambda_{\text {rest }}$ and the

\footnotetext{
Note, we do not subtract a pre-flare spectrum before measuring the bisector.
}

extrapolated excess C2826 continuum values are equal in intensity for BFP1 and BFP2. In the BFP2 spectrum (pink), the RWA component is much more prominent compared to the peak of the line at $\lambda_{\text {rest }}$ and is shifted less to the red. For BFP2, we subtract the extrapolation of $\mathrm{C} 2826$ and measure the $30 \%$ bisector velocity as $+9 \mathrm{~km} \mathrm{~s}^{-1}$, compared to a redshift of $+16 \mathrm{~km} \mathrm{~s}^{-1}$ for the peak of the RWA emission. The Mg II $\lambda 2791$ bisector velocity is a factor of 4 larger than the Fe II $\lambda 2814.45$ bisector velocity, which is due to the much brighter and broader RWA component in the $\mathrm{Mg}$ II line.

Figure 5 shows the observations for BFP1 and BFP2 for the Fe II $\lambda 2832.39$ line, which is a stronger line than Fe II $\lambda 2814.45$ in the flare, but has several other lines (Fe I and Ti II) to the red of $\lambda_{\text {rest }}$. In Figure 5 we also indicate the wavelengths that correspond to velocities of $\lambda-\lambda_{\text {rest }}=16 \mathrm{~km} \mathrm{~s}^{-1}$ and $\lambda-\lambda_{\text {rest }}=32 \mathrm{~km} \mathrm{~s}^{-1}$ by vertical lines, which identifies similar wavelength positions of the RWA peaks as for Fe II $\lambda 2814.45$.

We define the quantity $I_{\mathrm{RWA}} / I_{\lambda_{\text {rest }}}$ as the ratio of the peak intensity of the RWA component to the peak of the $\lambda_{\text {rest }}$ component (see also Liu et al. 2015). For the BFP2 spectrum (pink spectrum), $I_{\mathrm{RWA}} / I_{\lambda_{\text {rest }}} \sim 2 / 3$ for the Fe II $\lambda 2814.45$ line, whereas for the Fe II $\lambda 2832.39$ line, $I_{\mathrm{RWA}} / I_{\lambda_{\text {rest }}} \sim 1$, and the value of $I_{\mathrm{RWA}} / I_{\lambda_{\text {rest }}} \sim 1.7$ for the $\mathrm{Mg}$ II triplet line (Figure 3 ). For the BFP1 spectrum, the $I_{\mathrm{RWA}} / I_{\lambda_{\text {rest }}} \ll 1$ for Fe II $\lambda 2814.45$ whereas for the Fe II $\lambda 2832.39$ line, $I_{\mathrm{RWA}} / I_{\lambda_{\text {res }}} \sim 0.5$, and for the $\mathrm{Mg}$ II triplet line $I_{\mathrm{RWA}} / I_{\lambda_{\text {res }}} \sim 0.6$. The RWA component is more intense compared to the $\lambda_{\text {rest }}$ component for lines that are more optically thick, and when the RWA peak is shifted further to the red it has a lower intensity. In Section 5.6 we test our model predictions for the appearance of two emission line components (RWA and $\lambda_{\text {rest }}$ ) and the variation in relative brightness of these line components between Fe II $\lambda 2814.45$ and Fe II $\lambda 2832.39$.

\section{Radiative-hydrodynamic Flare Modeling}

\subsection{RADYN Flare Model Setup}

The high spatial resolution measurements of continuum intensity and chromospheric line profiles from IRIS, combined with constraints from RHESSI on the parameters of nonthermal electron beams, motivate new RHD flare models. We perform 1D plane-parallel, RHD flare modeling with the RADYN code (Carlsson \& Stein 1992, 1994, 1995, 1997, 2002) using the updated flare version described in Allred et al. (2015) to simulate flare heating from a nonthermal electron beam, in order to determine if the models give consistent results with the new IRIS data. We refer the reader to Allred et al. (2015) for a detailed description of the flare modeling method.

As our starting model atmosphere, we use a semi-circular loop of half-length $10 \mathrm{Mm}$ that corresponds to the QS.SL.HT model from Allred et al. (2015), which has an apex electron density and temperature of $8 \times 10^{9} \mathrm{~cm}^{-3}$ and $3.2 \mathrm{MK}$, 

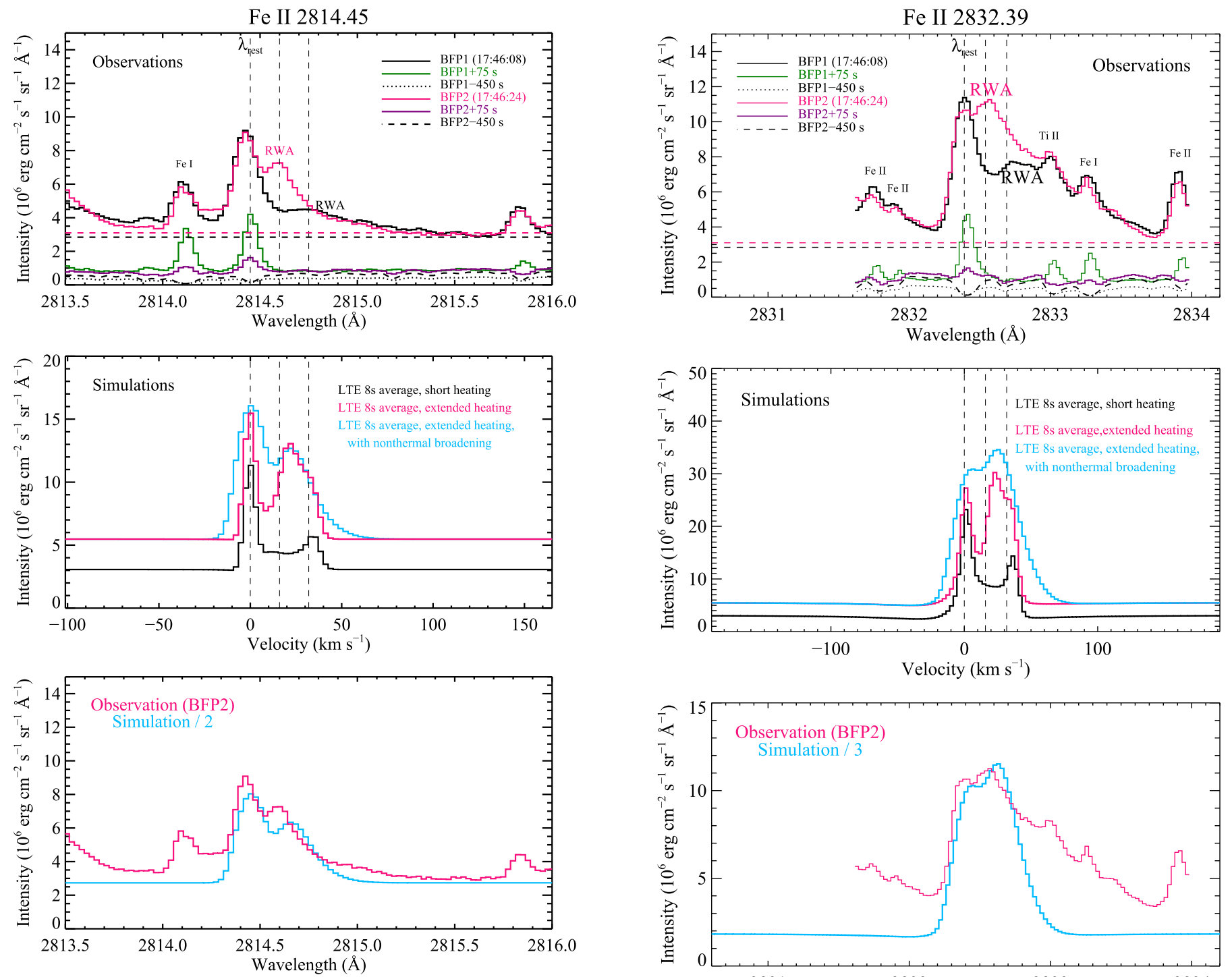

Figure 4. (Top) Fe II $\lambda 2814.45$ profiles for the same times and locations as in Figure 3. "RWA" indicates the locations of the peaks of the red wing asymmetry line components. The three vertical dashed lines show the rest wavelength for Fe II $\lambda 2814.45, \lambda-\lambda_{\text {rest }}=16 \mathrm{~km} \mathrm{~s}^{-1}$, and $\lambda-\lambda_{\text {rest }}=32 \mathrm{~km} \mathrm{~s}^{-1}$; these velocities identify the peaks of the RWA components for BFP2 and BFP1, respectively. The rest wavelength component of Fe I $\lambda 2814.11$ (Nave et al. 1994) is indicated in the top panel. The black and pink horizontal dashed lines are the values of C2826 extrapolated to this wavelength range. The dotted and dashed spectra indicate the pre-flare. (Middle) LTE Fe II $\lambda 2814.45$ profiles averaged over the first $8 \mathrm{~s}$ of the 5F11 model: the short $(4 \mathrm{~s})$ heating model (black) and the extended (15 s) heating model (pink). The light blue spectrum is the average of the first $8 \mathrm{~s}$ of the extended $5 \mathrm{~F} 11$ heating run with a nonthermal broadening parameter of $\xi=7 \mathrm{~km} \mathrm{~s}^{-1}$ included at heights $z>500 \mathrm{~km}, \xi=2 \mathrm{~km} \mathrm{~s}^{-1}$ included at $z<500 \mathrm{~km}$, and a variable value of $\xi(t)$ included in the CC (see text; Table 4). The same vertical dashed lines in the top panel are reproduced in the bottom panel. (Bottom) Simulation of Fe II $\lambda 2814.45$ with nonthermal broadening compared to the observation of BFP2 from the top panel. The model intensity has been reduced by half. The 5F11 model averaged over the first $8 \mathrm{~s}$ reproduces two spectrally resolved Fe II $\lambda 2814.45$ emission components with the same relative peak intensity as in the observations.

respectively. This starting atmosphere is closest to the plage environment where the majority of the high-thresh emission is observed in this flare (Figure 1), and the model loop length is consistent with the RHESSI hard X-ray footpoint separation in the early impulsive phase (Battaglia et al. 2015). In this preflare atmosphere, the transition region occurs at $z \sim 1150 \mathrm{~km}$,
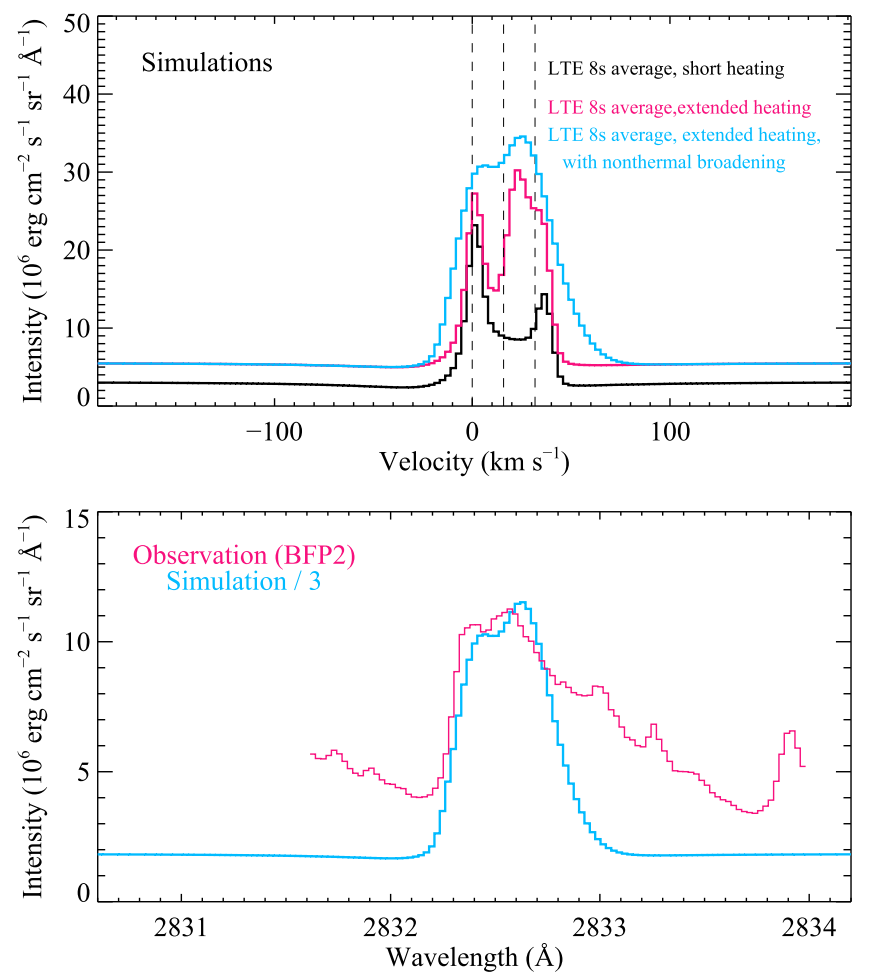

Figure 5. Same as Figure 4 but for Fe II $\lambda 2832.39$. Note the enlarged wavelength range. Other emission lines in this range are indicated: Fe II $\lambda 2833.92,2831.76,2831.92$ (Nave \& Johansson 2013), Fe I $\lambda 2833.27$ (Nave et al. 1994), and Ti II $\lambda 2832.99$ (I. Zapadlik et al. 2016, private communication). The two emission components in the 5F11 model averaged over the first $8 \mathrm{~s}$ are adequately reproduced with the correct relative intensity but are both brighter than the observations.

where $z=0$ is defined as the height where $\tau_{5000}=1$ at $t=0$. Carlsson et al. (2015) modeled Mg II lines from IRIS and concluded that plage regions have deeper transition regions due to the conductive flux from a hot, dense corona.

The equations of conservation of mass, momentum, energy, charge are solved together with the rate equations and the equation of radiative transfer on an adaptive grid scheme from Dorfi \& Drury (1987). The non-LTE (NLTE) problem is solved in RADYN using the technique of Scharmer (1981) and Scharmer \& Carlsson (1985) for three model atoms: a hydrogen atom (six levels including $\mathrm{H}$ II), a helium atom (five levels for He I, three levels for He II, and He III), and a singly ionized calcium ion (six levels including the Ca III ground state), giving a total of 22 bound-bound (b-b) and 19 bound-free (b-f) transitions calculated in detail. The optically thin loss function from Kowalski et al. (2015b) accounts for other b-b transitions 
not treated in detail, but excludes several ions that are likely optically thick at low temperature near $T \sim 10,000 \mathrm{~K}$ (see Section 7). Everything else is kept the same as in Allred et al. (2015) except that we have excluded the Mg II ion from the detailed radiative transfer and cooling rates. The proper calculation of the $\mathrm{Mg}$ II $h$ and $k$ lines during flares requires a prescription for several important effects that are not yet included in the RADYN code, such as overlapping b-b transitions and partial frequency redistribution, which may be affected by elastic collisions with the electron beam (Hawley et al. 2007). The cooling rate from the $\mathrm{Mg}$ II ion can be important for flare atmospheres (Avrett et al. 1986) but modifying RADYN to include overlapping transitions and partial redistribution is outside the scope of this work.

Wavelengths where the continuum is calculated in detail have been added at $\lambda=2826 \AA$ in the NUV and $\lambda=1332$, 1358, 1389, $1407 \AA$ in the FUV in order to compare to observed intensity measurements from IRIS. The continuum emission in the NUV calculated in RADYN includes all possible continua except for the opacity from the absorption wings of $\mathrm{Mg}$ II, which extend through the entire IRIS NUV wavelength range. We use the RH code (Uitenbroek 2001) to include the $\mathrm{Mg}$ II $h+k$ b-b opacities in the far wing at several times in the dynamic simulation at $\lambda=2826 \AA$ using a method discussed in Section 5.5. Five $\mu$ values are calculated in the models $(\mu=0.05,0.23,0.5,0.77$, and 0.95$)$; we compare the model values at $\mu=0.77$ which is closest to the observations of the flare $(\mu=0.82)$.

\subsection{Flare Heating Inputs}

The brightest hard X-ray RHESSI footpoints (Battaglia et al. 2015; Kleint et al. 2016) are cospatial with the brightest continuum emitting footpoints in the SOL2014-03-29T17:48 flare in Figure 1. To model the flare heating at these locations, we use a state-of-the-art prescription for energy deposition from a nonthermal electron beam which has been included in the RADYN flare code (Allred et al. 2015). The energy deposition rate is determined from the solution to the steadystate Fokker-Planck equation given a power-law of nonthermal electrons injected at the top of the model atmosphere. The Fokker-Planck solver has been adapted from McTiernan \& Petrosian (1990) and is available at http://hesperia.gsfc.nasa. gov/hessi/modelware.htm. The power-law parameters at the top of the model loop are chosen to be $E_{c}=25 \mathrm{keV}$ and $\delta=4.2$, which are consistent within the uncertainty range of the thick-target modeling presented in Kleint et al. (2016).

An important input for the RHD models is the energy flux density in nonthermal electrons, which cannot unambiguously be obtained from RHESSI data alone because the width of flare ribbons is known to be smaller than the resolution of RHESSI (Krucker et al. 2011; Sharykin \& Kosovichev 2014; Jing et al. 2016). We use the high spatial resolution of SJI 2832 to estimate a flare footpoint area for the calculation of the nonthermal energy flux. Kleint et al. 2016 derived an energy flux of $3.5 \times 10^{11} \mathrm{erg} \mathrm{cm}^{-2} \mathrm{~s}^{-1}$ for the bright hard X-ray source at 17:46:15-17:46:25 UT in the SW plage ribbon using an unresolved width of $1^{\prime \prime}$, which gives an area of $2.4 \times 10^{16}$ $\mathrm{cm}^{2}$ for the source (see also Judge et al. 2014). The high-thresh area $^{9}$ in the SW plage ribbon in SJI 2832 corresponds to $\lesssim 5 \times 10^{16} \mathrm{~cm}^{2}$, which is an upper limit because the cadence of

\footnotetext{
9 Areas are de-projected using the $\mu$ value of 0.82 for these observations.
}
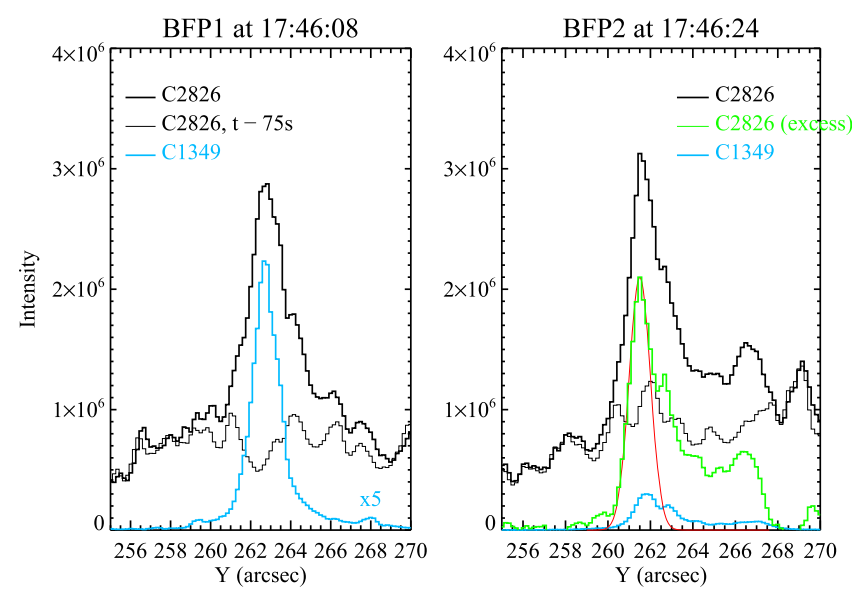

Figure 6. Spatial profiles from the $\mathrm{C} 2826$ and $\mathrm{C} 1349$ continuum regions for BFP1 and BFP2 in the bottom panel of Figure 1. For the NUV, the exposure time for the left panel is $8 \mathrm{~s}$ and the exposure for the right panel is $2.4 \mathrm{~s}$. The excess NUV intensity profile is shown in the right panel with a Gaussian fit (FWHM $\sim 1$ !' 2 ) to the leading edge of the profile; therefore, the leading edge of the NUV continuum emission is adequately resolved with IRIS at this location.

the observations is not fast enough to determine which areas are newly formed flare areas and which areas correspond to decaying emission. In the spatial profile of the excess C2826 (Figure 6, right panel), there are several peaks in the wake of the brightest part of the ribbon. A Gaussian with FWHM of 1 ". 2 can account for the "leading edge" (Isobe et al. 2007; Krucker et al. 2011) of the flare ribbon. Using the high-thresh area gives an acceptable lower limit on the flux of $1.5 \times 10^{11}$ erg cm $\mathrm{cm}^{-2} \mathrm{~s}^{-1}$, which is likely a factor of $\sim 2$ too low because the leading edge is the relevant area to divide into the hard $\mathrm{X}$-ray power.

If we use the area of BK2830 $\left(A \sim 1.5 \times 10^{16} \mathrm{~cm}^{2}\right.$ at FWHM intensity) at $(x, y)=(519 ! .5,263$ !" 8$)$ in Figure 1 (bottom), the flux would be $\geqslant 5 \times 10^{11} \mathrm{erg} \mathrm{cm}^{-2} \mathrm{~s}^{-1}$. As discussed by Battaglia et al. (2015), the RHESSI 35-100 keV images at 17:46 (SJI 2832 \#173) show two sources corresponding to the umbral and plage ribbons, and thus it is reasonable to assume that the spatially integrated nonthermal power originates from some flaring area that is associated with both ribbons and the RHESSI emission from the SW ribbon may include more flare area than BK2830. The high-thresh area for the mid peak phase includes area from both ribbons (see Figure 1, top panel) while the area of high thresh and hard X-ray emission are comparable (within a factor of 2), suggesting that the high thresh is a reasonable proxy for the hard X-ray RHESSI emission in this flare.

Thus, we consider a range of nonthermal energy flux for our modeling to bracket the (spatially averaged) values in the plage flare ribbon from Kleint et al. (2016): a model with a flux of $F_{\mathrm{NT}}=10^{11} \mathrm{erg} \mathrm{cm}^{-2} \mathrm{~s}^{-1}$ (F11; discussed in Kuridze et al. 2015 ) and a high beam flux model with $F_{\mathrm{NT}}=5 \times 10^{11}$ erg cm ${ }^{-2} \mathrm{~s}^{-1}$ (5F11). We note that lower values of the flux of $2-5 \times 10^{10} \mathrm{erg} \mathrm{cm}^{-2} \mathrm{~s}^{-1}$ have been inferred for this flare (e.g., Battaglia et al. 2015); see Heinzel et al. (2016) for RHD modeling of these flux levels using the Flarix code.

The nonthermal beam energy deposition duration is chosen to be $20 \mathrm{~s}$ for the F11 model. For the 5F11 model, we choose a short duration of flare heating for $4 \mathrm{~s}$ and an extended duration of heating for $15 \mathrm{~s}$. Rubio da Costa et al. (2016) used the derivative of the GOES1-8 $\AA$ light curve to infer heating 
timescales of 8-20 s at times around 17:46. However, we note that there are hard X-ray variations in the RHESSI light curve in Figure 2 that occur on shorter timescales, and Penn et al. (2016) show demodulated RHESSI light curves ( $1 \mathrm{~s}$ integration times) for another flare that exhibit short duration hard X-ray events with FWHM durations of only $4-5 \mathrm{~s}$. Thus, we explore a range of heating durations (4-15 s) for the 5F11 simulation. We use a constant heating profile and power-law index over the heating durations.

In the 5F11 model, shocks develop in the chromosphere (Section 5.4) and the time-steps become unmanageably small due to a radiative instability near the upper, lower density shock (see Kennedy et al. 2015, for a detailed analysis of this atmospheric region). At $t=3 \mathrm{~s}$ we adjust the second derivative of the adaptive grid weights to weight toward the higher density shock, and we decrease the accuracy of the minor level populations (see Kowalski et al. 2015b). These adjustments allow the 5F11 simulation to progress with larger time-steps. After $4 \mathrm{~s}$ of heating in the 5F11 model and $20 \mathrm{~s}$ of heating in the F11 model, the atmosphere is allowed to relax for $9 \mathrm{~s}$ and $60 \mathrm{~s}$, respectively, but the gradual phase evolution is not analyzed here.

We also analyze a coronal heating simulation without energy deposition from an electron beam. We simulate the atmospheric response to an energy flux of $10^{11} \mathrm{erg} \mathrm{cm}^{-2} \mathrm{~s}^{-1}$ deposited uniformly $\left(Q=125 \mathrm{erg} \mathrm{cm}^{-3} \mathrm{~s}^{-1}\right)$ over the upper $7.5 \mathrm{Mm}$ of the model corona. The heating duration for this model is $5 \mathrm{~s}$, and produces a corona with a temperature of $T \sim 30 \mathrm{MK}$. This simulation is used for a comparison of the NUV continuum emission and line profiles that are produced from a large conductive flux into the chromosphere, as done by other authors (e.g., Emslie \& Nagai 1985; Reep et al. 2016). A flux of $10^{11} \mathrm{erg} \mathrm{cm}^{-2} \mathrm{~s}^{-1}$ is 50 times larger than the value of the conductive flux from the heated corona in this flare found at the locations without detectable RHESSI hard X-ray emission (Battaglia et al. 2015).

The modeling analysis is divided into the following subsections: in Section 5.3, we calculate the excess NUV continuum intensity and $30 \%$ bisector velocity for the $\mathrm{H} \alpha$ line. The continuum quantities can be directly compared to the observations, and the $\mathrm{H} \alpha$ bisector is used as a proxy to what we expect for an optically thick line like the $\mathrm{Mg}$ II lines in the NUV. In Section 5.4 we summarize the hydrodynamics in the 5F11 simulation, which most adequately explains the observed properties of BFP1 and BFP2. In Section 5.5, we discuss the origin of the NUV continuum emission in the 5F11 model. In Appendix A, we discuss the upper photospheric heating and optical continuum emission in the 5F11 model. In Sections 5.6 and 5.7, we analyze the physical processes that reproduce the Fe II line profiles from Section 4.2.1. We analyze two time steps in the 5F11 model in detail at $t=1.8 \mathrm{~s}$ and $3.97 \mathrm{~s}$, and we also consider the differences in the model predictions at $t>4 \mathrm{~s}$ in the short heating run ( $4 \mathrm{~s}$ heating duration) and the extended heating run (15 $\mathrm{s}$ heating duration).

\subsection{Model Observables}

For the model runs, we calculate an excess C2826 continuum intensity and $30 \%$ line bisector for comparison to the observations of BFP1 and BFP2 (Section 4). The excess continuum values are calculated by subtracting the pre-flare model spectrum.
Table 2

Model Observables

\begin{tabular}{lccc}
\hline \hline Time & $\begin{array}{c}\text { Excess C2826 } \\
(\text { RADYN) }\end{array}$ & $\begin{array}{c}\text { Excess C2826 } \\
(\mathrm{RH})\end{array}$ & $\begin{array}{c}\text { H } \alpha 30 \% \text { Bisector } \\
\text { Velocity }\left(\mathrm{km} \mathrm{s}^{-1}\right)\end{array}$ \\
\hline $5 \mathrm{~F} 11$ & & & \\
\hline $\begin{array}{l}t=1.8 \mathrm{~s} \\
t=3.97 \mathrm{~s}\end{array}$ & 2.2 & 2.4 & $\sim 15$ \\
$\begin{array}{l}t=15 \mathrm{~s} \text { (extended } \\
\text { heating) }\end{array}$ & 5.1 & 5.4 & $\sim 30$ \\
$\begin{array}{l}t=0-8 \mathrm{~s} \text { (short } \\
\text { heating) }\end{array}$ & 1.2 & 4.1 & $\sim 10$ \\
$\begin{array}{l}t=0-8 \mathrm{~s} \text { (extended } \\
\text { heating) }\end{array}$ & 3.9 & $\ldots$ & $\ldots$ \\
\hline $\begin{array}{l}\text { F11 } \\
\quad\end{array}$ & $\ldots .7$ & $\ldots$ \\
$\begin{array}{l}t=3 \mathrm{~s} \\
t=18 \mathrm{~s}\end{array}$ & 0.64 & 0.78 & $\sim 0$ \\
\hline $\begin{array}{l}\text { Coronal heating } \\
\text { model }\end{array}$ & 0.7 & 1 & $<5$ \\
\hline \begin{tabular}{l}
$t=3 \mathrm{~s}$ \\
\hline
\end{tabular} & & & \\
\hline
\end{tabular}

Note. The excess $\mathrm{C} 2826$ values are the excess intensity values in units of $10^{6} \mathrm{erg} \mathrm{cm}^{-2} \mathrm{~s}^{-1}$ s.r. $^{-1} \AA^{-1}$. The pre-flare intensity from the RADYN calculation at $2826 \AA$ is $1.4 \times 10^{6} \mathrm{erg} \mathrm{cm}^{-2} \mathrm{~s}^{-1}$ s.r. $^{-1} \AA^{-1}$. The pre-flare intensity from the RH calculation including the $\mathrm{Mg}$ II $h+k$ wing opacity is $8.4 \times 10^{5} \mathrm{erg} \mathrm{cm}^{-2} \mathrm{~s}^{-1}$ s.r. $^{-1} \AA^{-1}$. A value of “..." means that these values were not necessary to calculate in this work.

The model observables are summarized in Table 2. For the excess C2826 continuum intensity, we first discuss the excess from the RADYN calculation (column 2), which does not include the $\mathrm{Mg}$ II wing opacity. In Section 5.5, we use the RH code to include the $\mathrm{Mg}$ II level populations for a refined calculation of the excess C2826 at select time steps (column 3). The excess C2826 values with Mg II wing opacities are comparable to or larger than the excess C2826 values obtained directly from RADYN; these differences are discussed in Section 5.5.

\subsubsection{Excess Continuum Intensity}

The time-evolution of the excess C2826 from the RADYN simulation is shown in Figure 7 for the the 5F11 model (for the extended $15 \mathrm{~s}$ and short $4 \mathrm{~s}$ beam heating durations). The C2826 attains a value of $2.2 \times 10^{6} \mathrm{erg} \mathrm{cm}^{-2} \mathrm{~s}^{-1}$ s.r. $^{-1} \AA^{-1}$ after $1.8 \mathrm{~s}$, and thus the excess NUV continuum intensity of the brightest flare footpoints BFP1 and BFP2 (Section 4, Table 1) is achieved in the 5F11 simulation. The NUV continuum continues to brighten to $5 \times 10^{6} \mathrm{erg} \mathrm{cm}^{-2} \mathrm{~s}^{-1}$ s.r. ${ }^{-1} \AA^{-1}$ at $4 \mathrm{~s}$ in the $5 \mathrm{~F} 11$. After $5 \mathrm{~s}$, the NUV continuum decreases slowly for the extended heating model and decreases rapidly in the short heating model. The F11 produces an excess C2826 that is at least a factor of three lower at all times. The coronal heating model produces an excess C2826 of $\lesssim 4 \times 10^{5} \mathrm{erg} \mathrm{cm}^{-2} \mathrm{~s}^{-1}$ s.r. ${ }^{-1} \AA^{-1}$, which is nearly a factor of two lower than the value that the F11 attains at comparable times. The relationship between the values of the excess C2826 and the atmospheric response are discussed in detail in Section 5.5.

The IRIS integration times of the NUV spectra range from 2.4-8 s, which are long compared to the continuum brightness time-evolution in the 5F11 model. Accounting for an IRIS 


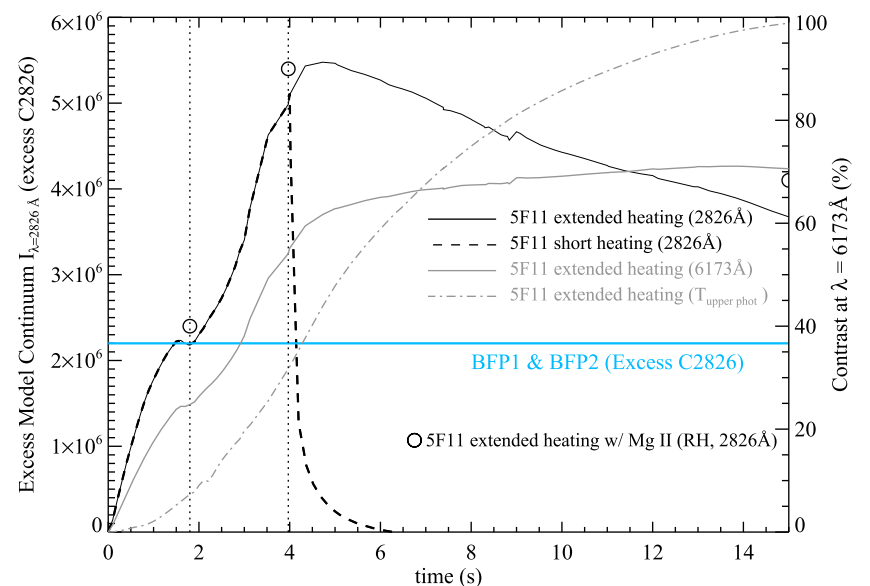

Figure 7. Light curve of the excess C2826 continuum intensity from the RADYN 5F11 simulation with short heating (dashed line) and extended heating (solid line). The excess continuum intensity at $\mathrm{C} 2826$ is shown for BFP1 and BFP2 (solid light blue horizontal line). The times analyzed in the early phase of the $5 \mathrm{~F} 11$ run are indicated by the two vertical dotted lines at $1.8 \mathrm{~s}$ and $3.97 \mathrm{~s}$. The open circles are the excess $\mathrm{C} 2826$ values calculated with the Mg II $h+k$ wing opacities at selected times (see text). The 5F11 model attains an excess NUV continuum intensity that is consistent with the spectral observations at $1.8 \mathrm{~s}$ and then continues to brighten as the chromospheric condensation increases in density and cools from $T \sim 25,000 \mathrm{~K}$ to $T \sim 10,000 \mathrm{~K}$. The evolution of the continuum after $4 \mathrm{~s}$ is markedly different if the beam heating continues or is turned off. The continuum contrast at $\lambda=6173 \AA$ (gray solid line) and the temperature increase in the upper photosphere (dashed-dotted line) are discussed in Appendix A; the temperature increase in the upper photosphere ranges from 4750 to $5680 \mathrm{~K}$ on the right axis.

integration time by averaging over the first $8 \mathrm{~s}$ gives excess C2826 values of $\sim 4 \times 10^{6} \mathrm{erg} \mathrm{cm}^{-2} \mathrm{~s}^{-1}$ s.r. ${ }^{-1} \AA^{-1}$ for the extended 5F11 heating run and $1.2 \times 10^{6} \mathrm{erg} \mathrm{cm}^{-2} \mathrm{~s}^{-1} \mathrm{~s}$. r. $^{-1} \AA^{-1}$ for the short $5 \mathrm{~F} 11$ heating run. An excess C2826 value of $\sim 4 \times 10^{6} \mathrm{erg} \mathrm{cm}^{-2} \mathrm{~s}^{-1}$ s.r. ${ }^{-1} \AA^{-1}$ is notably brighter than either BFP1 and BFP2; in Section 6, we compare the 5F11 prediction to the brightest pixels in the SJI 2832 image \#173 of BK2830 (Figure 1).

\subsubsection{Chromospheric Line Bisector}

The Mg II triplet lines in the NUV are not computed in the RADYN models, and thus we cannot directly compare the bisector velocities to the observations. As a proxy for the optically thick chromospheric emission lines, we use the $\mathrm{H} \alpha$ line which is calculated in RADYN. The line profiles for $\mathrm{H} \alpha$ at $t=1.8,3.97 \mathrm{~s}$ are shown in Figure 8 , and the $30 \%$ bisector velocity values are given in Table 2 (column 4). In the 5F11, a $\sim 30 \mathrm{~km} \mathrm{~s}^{-1}$ bisector velocity obtained from $\mathrm{H} \alpha$ is near the values obtained from $\mathrm{Mg}$ II $\lambda 2791.6$ (30\% bisector velocities of $\sim 40 \mathrm{~km} \mathrm{~s}^{-1}$; Section 4.2). A similar $30 \%$ bisector velocity measure of $\mathrm{H} \alpha$ is obtained in the coronal heating model. Large bisector redshifts are not obtained in the F11 model in the first $20 \mathrm{~s}$ of beam heating (see also Kuridze et al. 2015).

We conclude that the NUV continuum excess intensity and the bisector velocity of optically thick chromospheric lines are both adequately reproduced in the 5F11 model. In Sections 5.55.7 we use the $5 \mathrm{~F} 11$ model to describe the physical processes that produce the excess NUV continuum (C2826) intensity and the redshifted emission (RWA) component in the chromospheric Fe II lines in Figures 4-5.

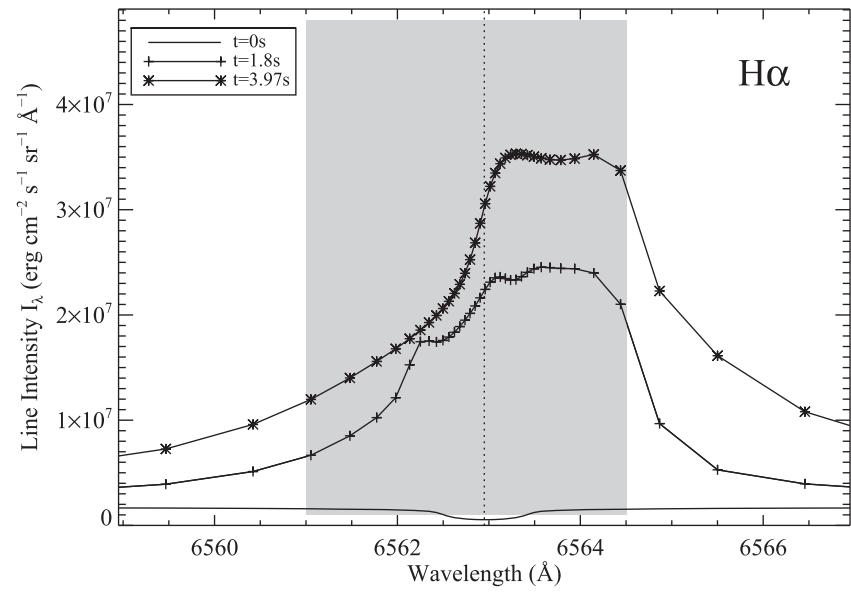

Figure 8. Instantaneous $\mathrm{H} \alpha$ line profiles from the 5F11 simulation. The rest wavelength is indicated by a vertical dotted line. This wavelength range is the same as in the Canfield \& Gayley (1987) figures, to facilitate comparison. The wavelength range of the IBIS spectral scans of $\mathrm{H} \alpha$ during this flare from Rubio da Costa et al. (2016) are indicated by the gray shaded region. Note, the default value of the microturbulence parameter in RADYN of $2 \mathrm{~km} \mathrm{~s}^{-1}$ is used for these profiles. A bisector velocity of $\sim 30 \mathrm{~km} \mathrm{~s}^{-1}$ is achieved at $t=3.97 \mathrm{~s}$, which is similar to the value obtained from the $\mathrm{Mg}$ II lines from IRIS.

\subsection{Dynamics of the $5 F 11 \mathrm{Model}$}

The atmospheric evolution of the $5 \mathrm{~F} 11$ is similar to the response of an $\mathrm{M}$ dwarf atmosphere to an F13 electron beam described in Kowalski et al. (2015b); and we refer the reader to Section 3.2 of Kowalski et al. (2015b) for a detailed description. Compared to the F13, the dynamical timescales of the 5F11 are longer and the magnitudes of the changes in the atmospheric parameters are generally less extreme. The main points are as follows: At $t=1.8 \mathrm{~s}$, explosive mass motions upward and downward have already developed from the formation of a $T \sim 10 \mathrm{MK}$ temperature plug at mid-chromospheric heights. The downward mass motions that originate in the chromosphere are referred to as the CC (chromospheric condensation) and the upward motions as chromospheric evaporation. The evaporation velocities in the lower corona $\left(n_{e}=10^{11}-10^{12} \mathrm{~cm}^{-3}\right)$ range between 150 and $500 \mathrm{~km} \mathrm{~s}^{-1}$. These were produced initially by a large increase in thermal pressure below the pre-flare transition region, and this is the evaporation of transition region material. On the lower side of this high temperature region, the large increase in thermal pressure drives material into a high density $\left(\rho_{\max }=1.7 \times 10^{-10} \mathrm{~g} \mathrm{~cm}^{-3}, T \approx 23,000 \mathrm{~K}\right) \mathrm{CC}$ with a height extent of approximately $25 \mathrm{~km}$.

The CC is a heated compression of the lower atmosphere that increases in density and decreases in temperature and speed as it accrues dense material at lower heights. A time step of the velocity and density evolution of the $5 \mathrm{~F} 11$ at $t=3.97 \mathrm{~s}$ (when the NUV continuum is nearly maximum) is shown in Figure 9. The evolution here can be compared directly to Figure 2 from Kowalski et al. (2015b) that describes the CC evolution in an F13 electron beam simulation (see also Appendix $\mathrm{C}$ here). The bottom panels of Figure 9 show the temperature, velocity, and mass density evolution at the location of the highest mass density in the $\mathrm{CC}$ for the $5 \mathrm{~F} 11$ model with extended heating. The downflowing velocities of the $\mathrm{CC}$ correspond to material with temperatures ranging from $T \approx 7500-5 \mathrm{MK}$ throughout the simulation, and the 

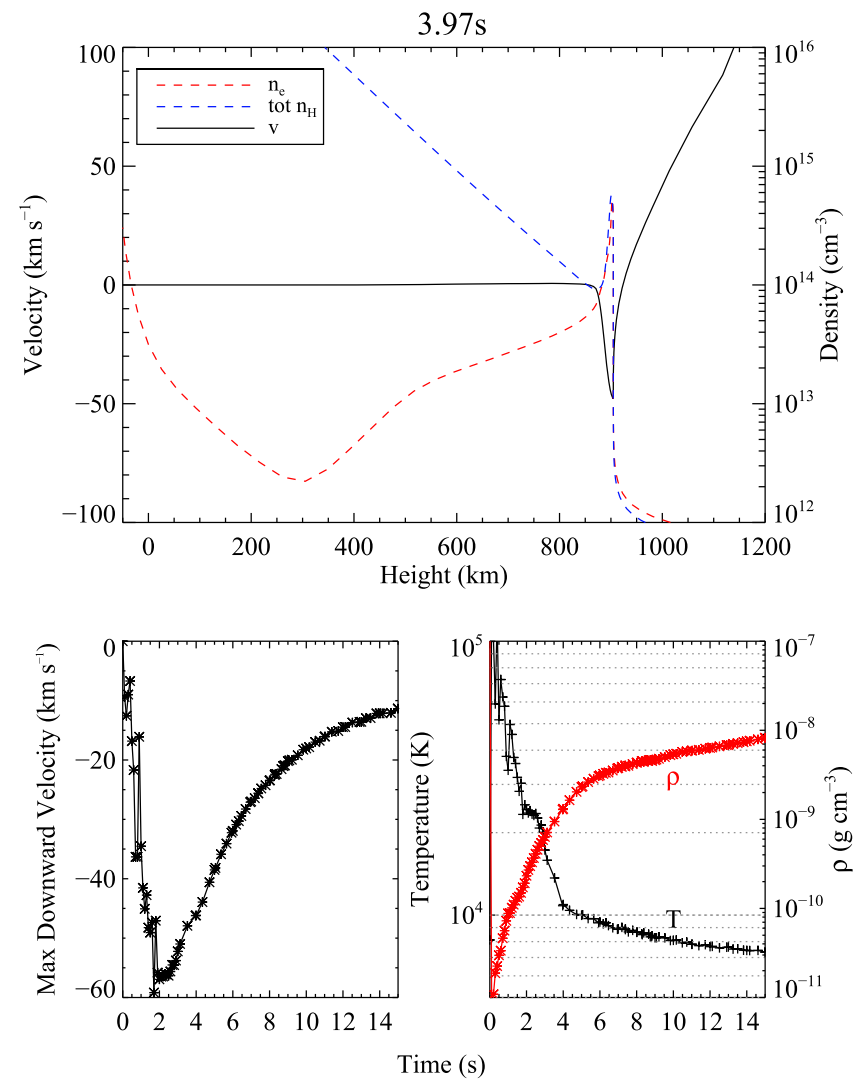

Figure 9. (Top) The density and velocity profiles of the lower atmosphere at $t=3.97 \mathrm{~s}$ in the 5F11 model. (Bottom, left) Time evolution of the maximum downward vertical velocity. (Bottom, right) Time-evolution of the temperature (black crosses) and density (red asterisks) at the location of the densest region of the $\mathrm{CC}$ for the extended 5F11 heating run. In the bottom right panel, horizontal dashed lines indicate the logarithmic tick marks on the left axis. The density increase in the top panel at $z \sim 900 \mathrm{~km}$ is the $\mathrm{CC}$, and the temperature and density evolution are anti-correlated.

temperature at the maximum density decreases from $T \sim 10^{5} \mathrm{~K}$ initially to $10^{4} \mathrm{~K}$ at $4 \mathrm{~s}$, as the CC increases in density. The condensation continues to cool below $T=10^{4} \mathrm{~K}$, increase in density, and decrease in speed after $4 \mathrm{~s}$ (for the extended heating run). The $\mathrm{CC}$ evolution qualitatively follows the analytic description from Fisher (1989) and the model atmosphere response to a softer $(\delta=7-8)$ electron beam over a longer $(\sim 100 \mathrm{~s})$ time in Kennedy et al. (2015). The 5F11 model is the first model to follow the development of a high flux beam-heated CC past the peak of the NUV response on short timescales.

\subsection{NUV Continuum Analysis of the 5F11 Model}

Figure 10 shows the evolution of the RADYN continuum spectrum in the 5F11 model. The flare spectrum consists of bright emission blueward of the Balmer limit at $\lambda=3646 \AA$. In this section, we discuss the atmospheric parameters and emission mechanisms that produce the emergent C2826 flare continuum intensity at $t=1.8$ and $3.97 \mathrm{~s}$; these times correspond to the mid-rise and near the peak, respectively (Figure 7). In Appendices A and B, we discuss the properties and evolution of continuum emission at other wavelengths in the NUV and optical that are indicated in Figure 10.

We calculate the contribution function to the emergent continuum intensity (Magain 1986; Carlsson 1998) and the optical depth at $\lambda=2826 \AA(\mu=0.77)$ for these

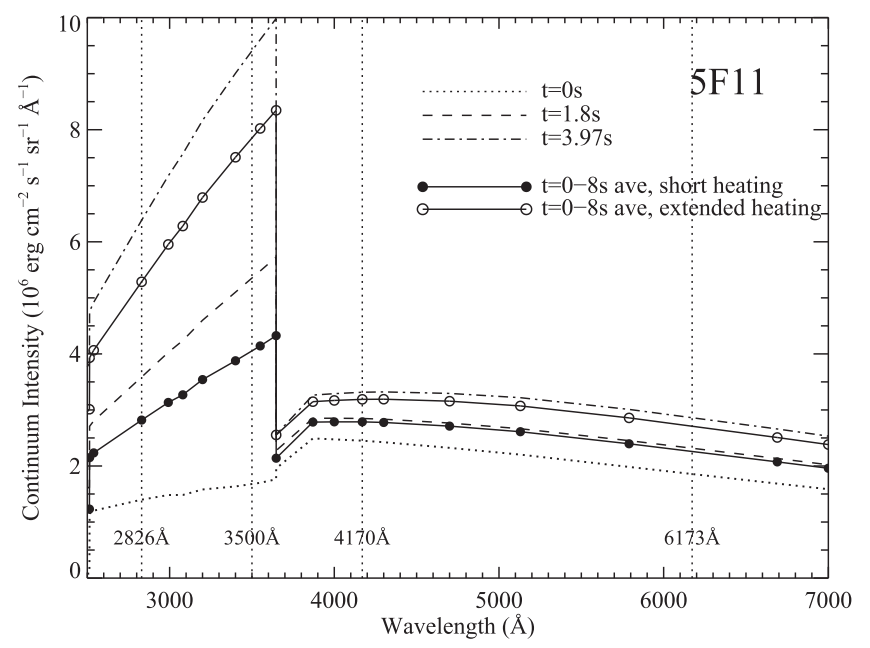

Figure 10. Continuum spectral evolution at selected times $(0 \mathrm{~s}, 1.8 \mathrm{~s}$, and $3.97 \mathrm{~s}$ ) in the $5 \mathrm{~F} 11$ and averaged over the first $8 \mathrm{~s}$ for the extended and short 5F11 model runs. Specific wavelengths discussed in the text and Appendix A are indicated by vertical dotted lines. The excess continuum emission at $\lambda<3646 \AA$ (the Balmer limit) is predominantly hydrogen recombination radiation emitted over low $\left(\tau_{\lambda} \lesssim 0.2\right)$ optical depth in two flaring layers, with relatively more emergent intensity from the $\mathrm{CC}$ at $3.97 \mathrm{~s}$ than at $1.8 \mathrm{~s}$. The ratio of excess NUV continuum to excess optical continuum is large (see Appendix A) due to the low optical depth at $T \sim 10,000 \mathrm{~K}$.

representative times and for $t=0 \mathrm{~s}$. Following Kowalski et al. (2015b) and Leenaarts et al. (2013b), we use the following form of the contribution function:

$$
C_{I}=\frac{d I_{\lambda}}{d z}=\frac{j_{\nu}}{\mu} e^{-\tau_{\nu} / \mu} \frac{c}{\lambda^{2}}
$$

where $j_{\nu}$ is the total continuum emissivity at a given height and $\tau_{\nu}$ is the optical depth at a given height obtained by integrating the total continuum opacity $\left(\chi_{\nu}(z)\right)$ over height. The NLTE spontaneous b-f emissivity and b-f opacity corrected for stimulated emission (Equations (1)-(7) of Mihalas 1978) are calculated using a six-level hydrogen atom with the population densities of each level given by the non-equilibrium ionization, NLTE populations from RADYN at each time step. The hydrogen f-f emissivity (where $B=S$ ) is included using the non-equilibrium ionization, NLTE proton density. Hydrogenlike helium has recombination edges in the NUV, and we include the NLTE b-f opacity and emissivity from these continua. Other continuum opacities and emissivities (from higher levels of hydrogen, $\mathrm{H}^{-}$and metals ${ }^{10}$ ) are calculated in LTE, as done internally in RADYN. We include the Thomson and Rayleigh scattering in the opacity and in the emissivity by calculating the angle-averaged intensity with a Feautrier solver from the RADYN code. These two processes are nonnegligible compared to the total emissivity during the flare only at heights where the NUV continuum contribution function is very small.

We use the RH code (Uitenbroek 2001) to include the Mg II wing opacity in the calculation of the emergent NUV continuum intensity, for a refined comparison to the excess

\footnotetext{
${ }^{10}$ Although the population of $\mathrm{H}^{-}$is not considered in the level population equation, its population density is calculated from the actual non-equilibrium, NLTE densities of electrons and neutral hydrogen atoms and included in the opacity in the equation of radiative transfer.
} 
C2826 obtained from the spectral observations. RH includes partial redistribution and overlapping opacities, which are appropriate for the Mg II $h+k$ line wings. Using a 3 level + continuum model $\mathrm{Mg}$ II+III atom, we calculate the NLTE population densities of the upper and lower levels of the $h$ and $k$ lines with statistical equilibrium. Thus, we are able to include the equilibrium ionization, NLTE Mg II $h+k$ wing opacity and emissivity at $\lambda=2826 \AA$ in Equation (2) with the nonequilibrium ionization, NLTE calculations of hydrogen and helium from RADYN.

The excess C2826 intensity values including the Mg II wing opacities are calculated by integrating Equation (2) and are given in Table 2 (column 3, "RH") at representative times: at $t=0,1.8,3.97,15 \mathrm{~s}$ in the $5 \mathrm{~F} 11$ model, at $t=3,18 \mathrm{~s}$ in the F11 run, and at $t=3 \mathrm{~s}$ in the coronal heating model. ${ }^{11}$ The C2826 intensity for the $t=0 \mathrm{~s}$ solar atmosphere is lower by $40 \%$ in the RH calculation than in the RADYN calculation, which is expected when the photospheric wing absorption profile is included. The RH calculation at $t=0 \mathrm{~s}$ falls between the range of values for the observed pre-flare intensity (Table 1) as expected since BFP1 occurs near a darker region and BFP2 occurs near brighter plage, but there is likely an error due to 3D effects and excluding Mg I in the NLTE calculation (Leenaarts et al. 2013b). For the excess flare intensity values, we find that the excess intensity in the F11 model at $t=18 \mathrm{~s}$ is $10^{6} \mathrm{erg} \mathrm{cm}^{-2} \mathrm{~s}^{-1}$ s.r. ${ }^{-1} \AA^{-1}$ and is higher than from the RADYN calculation by about $40 \%$, yet at least a factor of two lower than the observed C2826 values for BFP1 and BFP2. The refined excess C2826 values with Mg II $h+k$ opacities are shown in Figure 7 (open circles) for comparison to the timeevolution of the excess C2826 from RADYN. The excess C2826 values in the 5F11 model are 5\%-10\% larger in the RH calculation than in the RADYN calculation but follow the same trend. The 5F11 continuum prediction is closest to the observations at $t=1.8 \mathrm{~s}$, as concluded from the RADYN model continuum excess spectra without the Mg II wing opacity.

At $t \leqslant 0.05 \mathrm{~s}$ in the $5 \mathrm{~F} 11$, the contribution function for $\lambda=2826 \AA$ at photospheric and sub-photospheric heights decreases at the onset of the flare heating due to continuum dimming from high nonthermal collision rates in the chromosphere (Abbett \& Hawley 1999; Allred et al. 2005). After $t=0.05 \mathrm{~s}$, the maximum of the contribution function in the sub-photosphere (at $z \sim-40 \mathrm{~km}$ ) is $\sim 70 \%$ of the $t=0 \mathrm{~s}$ value due to opacity from increased thermal rates in the heated chromosphere and upper photosphere. The excess continuum intensity calculations that include the Mg II $h+k$ wing opacity ("RH" column 3 in Table 2) are thus larger than the excess continuum values without the wing opacity ("RADYN" column 2 in Table 2) because there is a smaller amount of decrease of (sub-)photospheric emission when Mg II $h+k$ wing opacity is included.

The contribution functions for the emergent intensity $\left(I_{\mu=0.77)}\right)$ at $\lambda=2826 \AA$ at $t=1.8$ and $3.97 \mathrm{~s}$ are shown in Figures 11 and 12 compared to the temperature and density structure at these two times. Following Kowalski (2016), we also calculate the normalized cumulative contribution

\footnotetext{
${ }^{11}$ For $t=1.8 \mathrm{~s}$ in the $5 \mathrm{~F} 11$ and $t=3 \mathrm{~s}$ in the coronal heating model, we have set the velocities in the atmosphere to $0 \mathrm{~km} \mathrm{~s}^{-1}$ to facilitate convergence. We do not expect this to affect the calculation of continuum wavelengths in the far wing of the line.
}
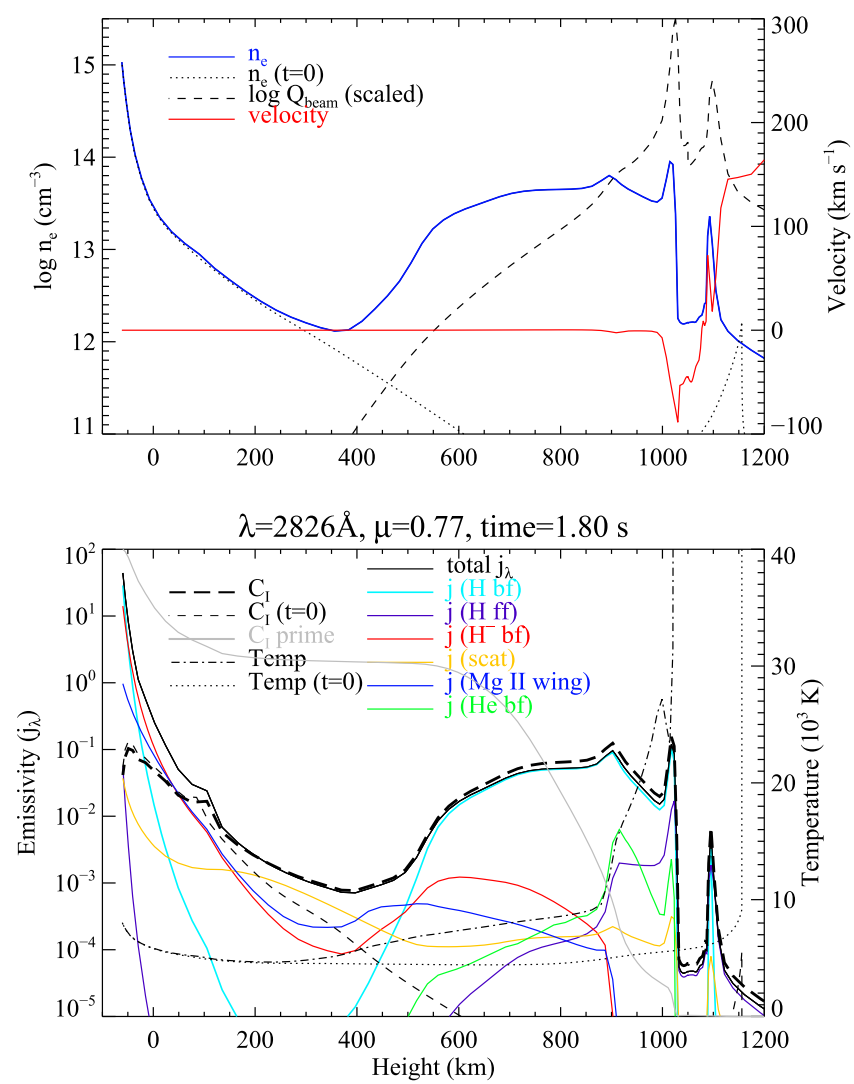

Figure 11. (Top) Atmospheric parameters at $t=1.8 \mathrm{~s}$. The volumetric beam heating is shown over 4.5 dex on the left axis. (Bottom) The $\lambda=2826 \AA$ contribution function at $\mu=0.77$ at $t=1.8 \mathrm{~s}$ compared to the temperature profile (right axis). The total emissivity and the emissivity for relevant atomic processes are shown as a function of height; the solid gray line is the cumulative contribution function $\left(\mathrm{C}_{I}^{\prime}\right)$, which ranges from 0 to 1 and is scaled linearly to the right axis. The pre-flare contribution function is shown as a dashed line. $j_{\text {scat }}$ includes Rayleigh and Thomson scattering. Spontaneous hydrogen b-f Balmer emissivity from the stationary flare layers from $z \sim 600-1000 \mathrm{~km}$ dominates the emergent NUV intensity at this time when the $\mathrm{CC}$ is $T \sim 25,000 \mathrm{~K}$.

function $C_{I}^{\prime}$ :

$$
C_{I}^{\prime}(z, \mu)=1-\frac{\int_{z \geqslant z_{\mathrm{lim}}}^{z=10 \mathrm{Mm}} C_{I}(z, \mu) d z}{\int_{z_{\lim }}^{z=10 \mathrm{Mm}} C_{I}(z, \mu) d z},
$$

where $z$ is the height variable (the height variable is defined as $z=0$ at $\tau_{5000}=1 ; z=10 \mathrm{Mm}$ corresponds to the top of the model corona), and the denominator is equal to the emergent intensity $I_{\lambda}(\mu)$ if $z_{\lim }$ corresponds to the height of the lowest grid point of the model atmosphere. The physical depth range parameter, $\Delta z$, is the height difference between $C_{I}^{\prime}=0.95$ and $C_{I}^{\prime}=0.05$ and thus quantifies the vertical extent of the atmosphere over which a majority of the emergent intensity is formed. ${ }^{12}$ The variation of $\Delta z(\lambda)$ is a proxy for comparing the optical depth among continuum wavelengths. $C_{I}^{\prime}(z)$ is also useful for determining the fraction of emergent intensity that originates from atmospheric layers above $z$. The $C_{I}^{\prime}(z, \mu)$ curves are shown in Figures 11 and 12 and linearly scaled from 0 to 1 on the right axis $\left(z_{\lim }=-60 \mathrm{~km}\right)$.

\footnotetext{
${ }^{12}$ In Kowalski et al. (2015b), the physical depth range of the CC was calculated as the FHWM of the contribution function within the CC.
} 

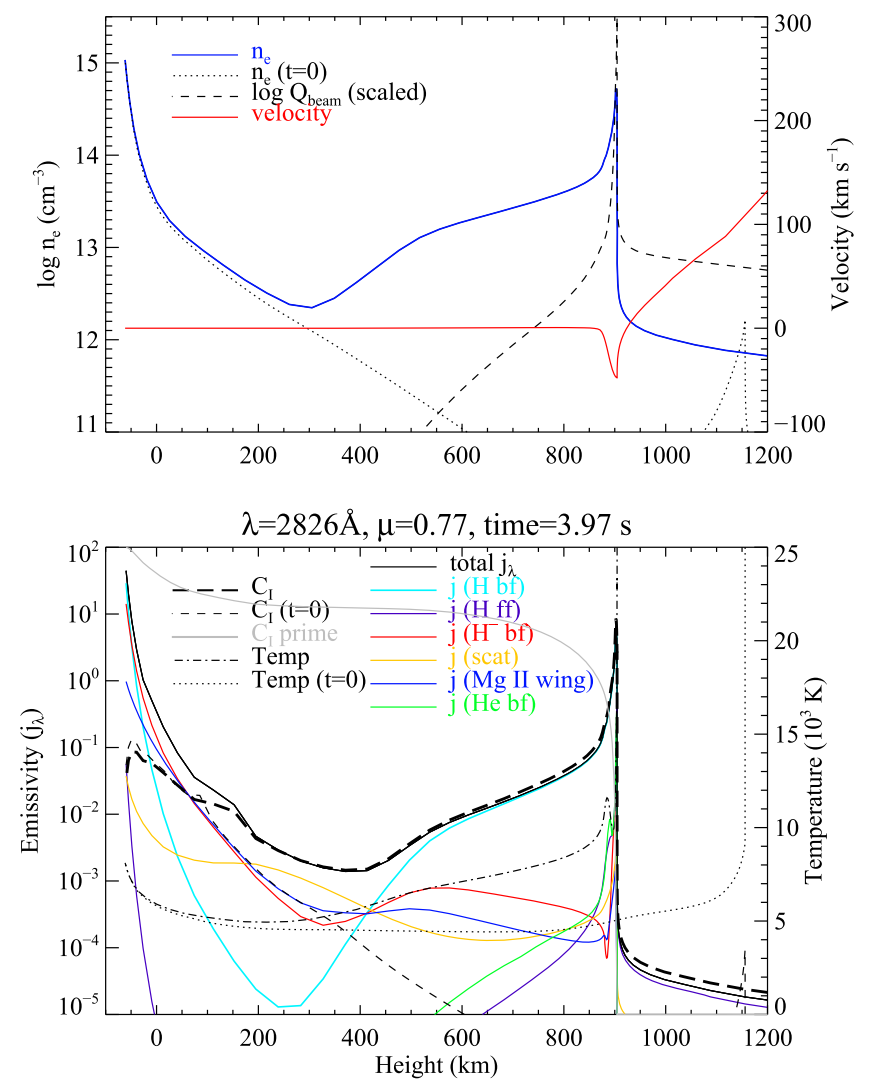

Figure 12. Same as for Figure 11 but at $t=3.97 \mathrm{~s}$. Spontaneous hydrogen Balmer b-f emissivity from a narrow height range in the $\mathrm{CC}$ at $z \sim 900 \mathrm{~km}$ dominates the emergent NUV continuum intensity at this time.

\subsubsection{The Origin of the NUV Continuum Intensity in the 5F11 Model}

The excess NUV continuum intensity (C2826) during the 5 F11 originates over low optical depth continuum-emitting atmospheric layers at $z>500 \mathrm{~km}$, which we refer to as the flare chromosphere. In Figures 11 and 12, the $C_{I}^{\prime}$ curves are flat from $z \approx 150-550 \mathrm{~km}$ showing that at these times there is not any contribution to the emergent NUV intensity over this height range. At $z<500 \mathrm{~km}$, there is not a relatively large amount of enhanced NUV continuum emissivity during the rise phase (from $t=0-4 \mathrm{~s}$ in Figure 7). Only at later times in the extended 5F11 simulation does the upper photospheric temperature increase (Appendix A) to produce a net increase in the height-integrated continuum emission at $\mathrm{C} 2826$ at $z<500 \mathrm{~km}$. For calculations of $\Delta z$ in the first $10 \mathrm{~s}$, we use $z_{\text {lim }}=500 \mathrm{~km}$ in Equation (3) since increased emissivity predominantly occurs in higher layers. The physical depth range for $\mathrm{C} 2826$ at $1.8 \mathrm{~s}$ as $\Delta z=390 \mathrm{~km}$, from $z \sim 630-1020 \mathrm{~km}$. At $3.97 \mathrm{~s}, \Delta z=160 \mathrm{~km}$, from $z \sim 750-905 \mathrm{~km}$. Thus the region producing the NUV continuum emission becomes narrower while peaking near $z \sim 900 \mathrm{~km}$ during the rise phase of the excess C2826 as it increases in brightness by a factor of $\sim 2$.

In the bottom panels of Figures 11 and 12, we show the emissivity for several atomic processes that are included in the contribution function for C2826. Where most of the excess NUV continuum intensity originates $(z>500 \mathrm{~km})$, the dominant process for the emission of NUV photons is hydrogen (Balmer, $n=2$ ) b-f emission. Although the optical depths increase during the flare, $\tau_{2826}$ attains a value of only 0.15 at $z=500 \mathrm{~km}$ at $t=1.8 \mathrm{~s}$ and increases to only 0.2 at $t=3.97 \mathrm{~s}$.
These values of the optical depth are comparable to the value obtained in Heinzel \& Kleint (2014) using the static F11 beam model calculations from Ricchiazzi \& Canfield (1983). The density increases in the $\mathrm{CC}$ at $t=3.97 \mathrm{~s}$ to $n_{\mathrm{H}, \max }=5 \times 10^{14}$ $\mathrm{cm}^{-3}$ but the optical depth at $\lambda=2826 \AA$ in the CC remains low at $\tau_{2826} \sim 0.07$. The radiation from the $\mathrm{CC}$ is optically thin because the relatively low column density for $n=2$ level of hydrogen, which predominantly determines the optical depth at this temperature, density, and wavelength, in the $\mathrm{CC}$ is only $2 \times 10^{15} \mathrm{~cm}^{-2}$. This value is far from the value that produces optically thick emission in the NUV $\left(3 \times 10^{17} \mathrm{~cm}^{-2}\right.$; Kowalski et al. 2015b). The physical depth range does not vary largely with wavelength over the NUV and optical wavelength regimes in the $5 \mathrm{~F} 11$ and the logarithm of the contribution function for C2826 traces the logarithm of the electron density profile and evolution (Figures 11 and 12), which are generally indicative of optically thin hydrogen recombination radiation from a flare atmosphere. ${ }^{13}$ Because the observed excess NUV continuum intensity is achieved in the 5F11 model (Figure 7), we conclude that the NUV continuum emission from BFP1 and BFP2 can be explained by Balmer continuum radiation from a flaring chromosphere with a CC that has a high density but low optical depth.

The flare-enhanced NUV continuum intensity primarily originates from two beam-heated flare layers with increased electron density at $z>500 \mathrm{~km}$ : downflowing $\mathrm{CC}$ layers and non-moving flare layers below the $\mathrm{CC}$. In the top panels of Figures 11 and 12, the downflowing (negative) velocities correspond to peaks in the electron density and in the C2826 contribution function (bottom panels); these are the cooler layers of the $\mathrm{CC}$ with $T \lesssim 25,000 \mathrm{~K}$ (the $\mathrm{CC}$ includes material with temperatures as high as several MK over the evolution, but only the high density cooler regions give rise to the continuum and NUV line emission). We define the vertical extent of the $\mathrm{CC}$ as the layers where the velocity is $v<-5 \mathrm{~km} \mathrm{~s}^{-1}$ and where $C_{I, 2826}^{\prime}>0.05$ (i.e., those that are visible in NUV continuum light). The $\mathrm{CC}$ is only about $\sim 25 \mathrm{~km}$ in vertical extent at these two times. There is also continuum emission that originates from the layers with nearly negligible velocity that extend from the bottom of the $\mathrm{CC}$ to several hundred $\mathrm{km}$ below the CC. There is a small upflow velocity of $<1 \mathrm{~km} \mathrm{~s}^{-1}$ that develops in these layers, but this is small and therefore not possible to robustly detect in the observations. We therefore refer to these flare layers as the stationary flare layers. Using Equation (3), we calculate that $\sim 90 \%$ and $20 \%$ of the emergent C2826 intensity originates from the stationary flare layers at $t=1.8$ and $3.97 \mathrm{~s}$, respectively. The increase in brightness from $1.8 \mathrm{~s}$ to the peak in Figure 7 is a result of the large increase in the $\mathrm{CC}$ contribution to the emergent C2826 intensity.

The relative contributions from the stationary and CC layers to the excess C2826 intensity is a combination of the evolution of the electron density and temperature in these two flare layers at $z>500 \mathrm{~km}$. In Table 3 , we give the representative physical parameters in the $\mathrm{CC}$ and in the stationary flare layers over time steps of the simulation. The representative electron density and temperatures in each flare layer are given as the C2826

\footnotetext{
3 Figures 2, 4, and 6 of Kowalski et al. (2015b) illustrate how the contribution function of Balmer continuum emission over large optical depth does not follow the electron density in a flare atmosphere with a much more dense CC than in the 5F11 model, and Figure 2 of Kowalski (2016) shows how the physical depth range of emergent continuum intensity is affected by large optical depth. See also Appendix C here.
} 
Table 3

Essential Parameters of the Lower Flare Atmosphere

\begin{tabular}{|c|c|c|c|c|c|c|c|c|c|c|c|}
\hline $\begin{array}{l}\text { Time } \\
\text { (s) }\end{array}$ & $\underset{(\mathrm{km})}{\Delta z}$ & $\begin{array}{c}z_{1} \\
(\mathrm{~km})\end{array}$ & $\begin{array}{c}z_{2} \\
(\mathrm{~km})\end{array}$ & $\begin{array}{c}z_{3} \\
(\mathrm{~km})\end{array}$ & $T \underset{(\mathrm{K})}{\left(z_{1}-z_{2}\right)}$ & $\begin{array}{c}T\left(z_{2}\right)(\mathrm{K}) \\
(\mathrm{K})\end{array}$ & $\underset{(\mathrm{K})}{T\left(z_{2}-z_{3}\right)}$ & $\begin{array}{c}n_{e}\left(z_{1}-z_{2}\right) \\
\left(\mathrm{cm}^{-3}\right)\end{array}$ & $\begin{array}{c}n_{e}\left(z_{2}-z_{3}\right) \\
\left(\mathrm{cm}^{-3}\right)\end{array}$ & $Q_{b}\left(z>z_{2}\right)$ & $Q_{b}\left(z>z_{3}\right)$ \\
\hline \multicolumn{12}{|c|}{ 5F11 Extended Heating Model } \\
\hline 1.8 & 390 & 632 & 1000 & 1020 & 10570 & 27170 & 24250 & $4.6 e+13$ & $7.4 \mathrm{e}+13$ & 0.85 & 0.30 \\
\hline 3.97 & 160 & 744 & 878 & 904 & 8790 & 10330 & 12000 & $4.8 e+13$ & $3.6 \mathrm{e}+14$ & 0.96 & 0.47 \\
\hline 7.0 & 50 & 741 & 766 & 794 & 8190 & 8520 & 10670 & $4.3 e+13$ & $4.6 e+14$ & 0.99 & 0.65 \\
\hline
\end{tabular}

Note. $\Delta z$ is the physical depth range at $\lambda=2826 \AA$; we used $500 \mathrm{~km}$ as the minimum height ( $z_{\text {lim }}$ in Equation (3)). $z_{1}$ is the bottom of the stationary flare layer where $C_{I}^{\prime}=0.95, z_{2}$ is the bottom of the chromospheric condensation where the downward gas speed falls below $5 \mathrm{~km} \mathrm{~s}^{-1}, z_{3}$ is the top of the chromospheric condensation where $C_{I}^{\prime}=0.05$. The temperature and density values are weighted by the continuum contribution function in each region of the atmosphere, and $Q_{b}$ is the fraction of beam energy flux deposited at heights greater than $z$.

contribution function-weighted values in the two flare layers in columns $6,8,9$, and 10 . The bottom extent of the stationary flare layers $\left(z_{1}\right)$ is defined as the height where $C_{I}^{\prime}=0.95$ (this height changes as a function of continuum wavelength), and layers just below the $C_{I}^{\prime}=0.95$ layer for $\lambda=2826 \AA$ are also heated but to a lesser extent such that comparable amounts of NUV continuum emission to the contributions in the $\mathrm{CC}$ and stationary flare layers are not produced at $z<500 \mathrm{~km}$. The value of $z_{2}$ is the division between the stationary and CC layers; the temperature at $z_{2}$ is given in column 7. $z_{3}$ is defined as the top of the CC with $v<-5 \mathrm{~km} \mathrm{~s}^{-1}$ and where $C_{I, 2826}^{\prime}=0.05$. Over the evolution of the simulation from $1.8 \mathrm{~s}$ to $3.97 \mathrm{~s}$, the $\mathrm{CC}$ increases in electron density from $7 \times 10^{13} \mathrm{~cm}^{-3}$ to nearly $4 \times 10^{14} \mathrm{~cm}^{-3}$. At $1.8 \mathrm{~s}$, the $\mathrm{CC}$ and stationary flare layers have comparable electron densities $\left(\sim 5-7 \times 10^{13} \mathrm{~cm}^{-3}\right)$ but at $3.97 \mathrm{~s}$, the electron density in the $\mathrm{CC}$ is nearly an order of magnitude larger than in the stationary layers and thus the relative amount of continuum emission is larger.

The ambient electron density and the temperature in the two flaring layers is determined by the evolution the beam heating rate, which is a function of the electron beam parameters and column mass evolution. In both flare layers, the beam energy deposition (shown in Figures 11 and 12) dominates the sources that contribute to the increase in internal energy at $z>550 \mathrm{~km}$ in the stationary flare layers and in the $\mathrm{CC}$ for temperatures $<25,000 \mathrm{~K}$. At the height corresponding to $T \sim 25,000 \mathrm{~K}$, there is a steep temperature gradient at the lower shock (Section 5.4) where conductive heating dominates. At $z \lesssim 550 \mathrm{~km}$, radiative heating from the absorption of photons in the Balmer continuum wavelength range dominates the sources that increase the internal energy.

In the last two columns, we give the fraction of beam energy deposited at heights greater than the stationary flare layers $\left(z>z_{2}\right.$; column 11) and heights greater than the $\mathrm{CC}\left(z>z_{3}\right.$; column 12) for the 5F11 model. Due to the increasing density in the corona (from ablation), a larger fraction of the beam energy deposition occurs at larger heights over time. By $t=3.97 \mathrm{~s}$, only $50 \%$ of the 5F11 beam energy flux is deposited at heights within the $\mathrm{CC}$ and below $\left(z<z_{3}\right)$ and is less at later times. The temperature and the electron density thus drop in the stationary flare layers (e.g., at $z \sim 750 \mathrm{~km}$ ) from $1.8 \mathrm{~s}$ to $3.97 \mathrm{~s}$. From 0 to $4 \mathrm{~s}$, almost all of the material in a $175 \mathrm{~km}$ region from $z \sim 900-1075 \mathrm{~km}$ has been compressed into a $25 \mathrm{~km}$ region (the CC) at $z \sim 900 \mathrm{~km}$. Due to the increasing density in the $\mathrm{CC}$ (from compression), an approximately constant fraction $(\sim 50 \%)$ of the beam energy deposition occurs in the descending, narrow height range of the $\mathrm{CC}$, and thus more energetic electrons heat at lower heights (Kennedy et al. 2015).

Following the analysis of Kennedy et al. (2015), we calculate the classical thick-target stopping depth (Emslie 1978) as a function of electron energy for several times in the 5F11. First, to confirm that the classical stopping depth formula gives reasonable values, we use the evolved 5F11 atmosphere at $3.97 \mathrm{~s}$ and calculate the Fokker-Planck energy deposition with $\delta=10$ and lower energy cutoffs of $E_{c}=25,35,50,85 \mathrm{keV}$ which are nearly monoenergetic beams with $E_{\text {ave }} \sim E_{c}$. Beam electrons with energies initially $E>50 \mathrm{keV}$ at $1.8 \mathrm{~s}$ and $E>80 \mathrm{keV}$ at $3.97 \mathrm{~s}$ heat the stationary flare layers, whereas lower energy beam electrons primarily heat the $\mathrm{CC}$ and the upward flows in the flare corona. As less of the beam energy is deposited in the stationary flare layers (column 11) and the CC becomes brighter from an order magnitude increase in density as the temperature decreases to $\sim 10,000 \mathrm{~K}$, most of the emergent intensity originates from the $\mathrm{CC}$ and the physical depth range of the continuum emission decreases from 390 to $160 \mathrm{~km}$.

\subsection{Fe II $\lambda 2814.45$ Modeling}

With hydrodynamic modeling, we can rigorously test the model predictions of the dynamics over the continuum-emitting layers by synthesizing flare chromospheric lines that have low optical depth and probe the temperatures $(T \sim 10,000 \mathrm{~K})$ of white-light continuum formation. In this section, we model the Fe II lines in LTE to show that the flare atmospheric evolution of the $5 \mathrm{~F} 11$ is qualitatively consistent with the resolved spectral components at $\lambda_{\text {rest }}$ and the RWA emission in the NUV spectra of BFP1 and BFP2 (Figures 3-5, Section 4.2.1).

We use the atomic data for the Fe II $\lambda 2814.45$ and Fe II $\lambda 2832.39$ lines from Sikström et al. (1999), Nave \& Johansson (2013), and Raassen \& Uylings (1998) using the National Institute of Standards and Technology database and the partition function from Halenka \& Grabowski (1984) to calculate the LTE level populations of the upper and lower levels of the transitions. The temperature, electron density, mass density, and velocity are obtained from each time step in the 5F11, F11, and coronal heating RADYN simulations at approximately $0.1-0.3 \mathrm{~s}$ intervals (thus, the LTE Fe II calculations include the non-equilibrium ionization, NLTE electron density at each time). We assume that scattering is not important for these lines, and therefore the LTE line emissivity and opacity can be written as Equations (2.69) and (3.87), respectively, from Rutten (2003). The rates for (stronger) Fe II lines in the NUV are collisionally dominated for the electron 

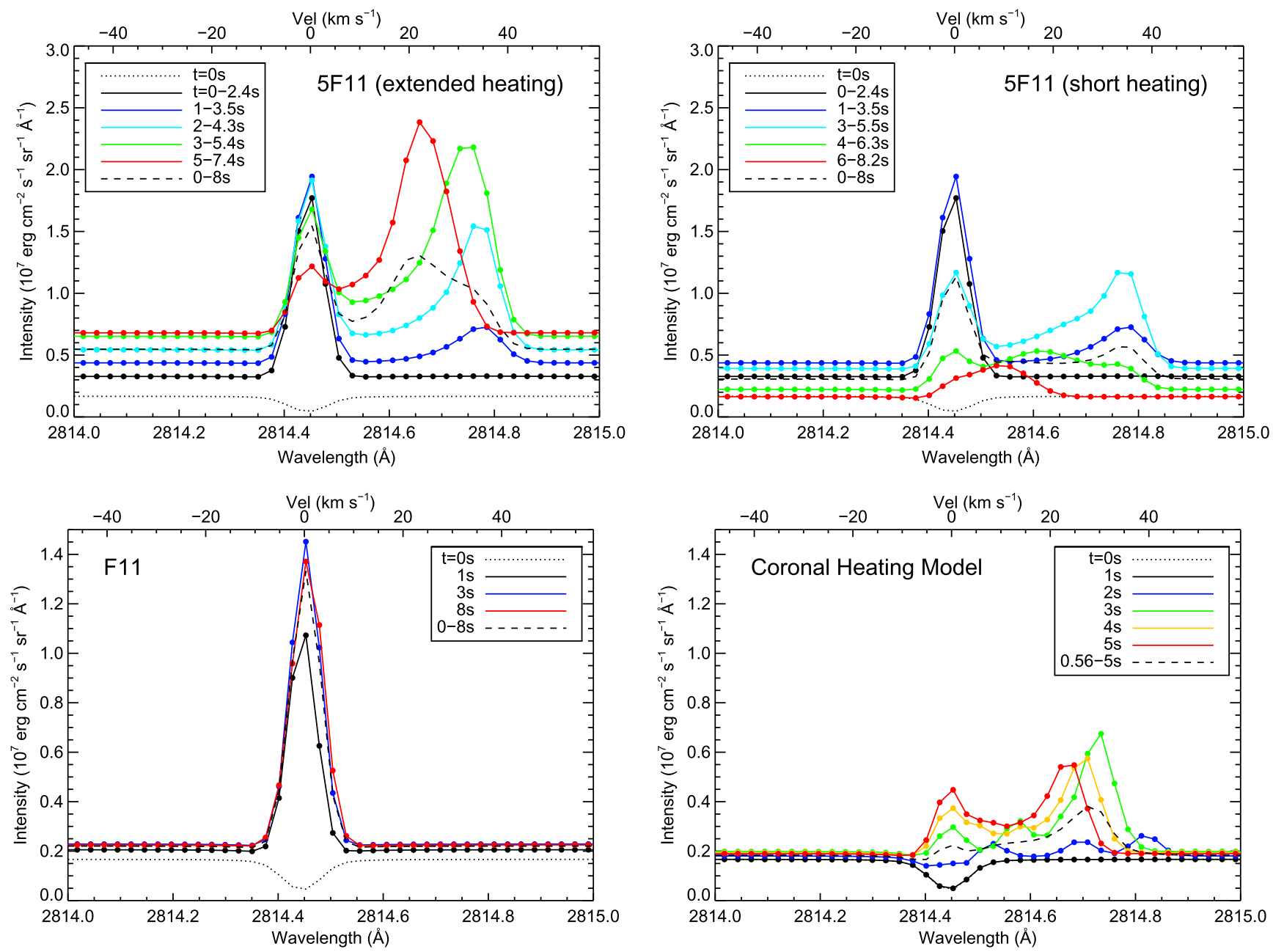

Figure 13. (Top) Evolution of the Fe II $\lambda 2814.45$ line for the $5 \mathrm{~F} 11$ model with extended heating (top left) and short heating (top right) averaged over select $2.4 \mathrm{~s}$ intervals. The preflare LTE calculation is shown as a dotted line, and the dashed curve is the $8 \mathrm{~s}$ exposure average spectrum from Figure 4 . (Bottom left) The evolution of instantaneous LTE Fe II $\lambda 2814.45$ profiles in the F11 simulation at selected times; the average over the first $8 \mathrm{~s}$ is shown as a dashed line. (Bottom right) The evolution of the instantaneous LTE Fe II $\lambda 2814.45$ profiles for the coronal heating model at selected times; the average over the first $5 \mathrm{~s}$ is shown as a dashed line. Note the small increase in the continuum emission and the smaller ranges on the $y$-axes in the bottom panels. Nonthermal broadening is not used in these calculations. Averaged over the heating, the F11 model produces no RWA intensity, the coronal heating model produces too much RWA intensity compared to intensity at the rest wavelength, and the 5F11 model produces the observed amount of RWA intensity relative to the intensity at the rest wavelength. The RWA intensity in the 5F11 model appears at large positive velocity and the peak shifts to bluer wavelengths as the CC decreases in velocity (Figure 9).

density of the quiet Sun (Judge et al. 1992); since the flare atmospheres increase the density and temperature, LTE is a plausible approximation for the minor Fe II lines (see also Section 7). The continuum emissivity and opacity from Section 5.5 are also included in the calculation (however, we exclude Mg II from these calculations). The time-averaged emergent intensity spectra are calculated for each model over a simulated IRIS exposure time of $8 \mathrm{~s}$ and are convolved with the instrumental resolution (FWHM $\sim 5.5 \mathrm{~km} \mathrm{~s}^{-1}$ ). For the $5 \mathrm{~F} 11$ model, we average the short $5 \mathrm{~F} 11$ heating run ( $4 \mathrm{~s}$ heating) over the first $8 \mathrm{~s}$ of the simulation, and we average the extended (15 s) $5 \mathrm{~F} 11$ heating run over the first $8 \mathrm{~s}$ of the simulation. We also calculate average spectra over $2.4 \mathrm{~s}$ intervals to compare to the shorter exposure times resulting from the IRIS AEC adjustment during the flare.

The $8 \mathrm{~s}$ average model intensity spectra for the Fe II $\lambda 2814.45$ line are shown as the black and pink curves for the short and extended heating runs, respectively, in the middle panel of Figure 4. The models produce a broad RWA component and a narrower component at $\lambda_{\text {rest }}$, as in the observations (top panel of Figure 4). The value of $I_{\mathrm{RWA}} / I_{\lambda_{\text {rest }}}$ from the models are $\sim 1 / 3$ and $2 / 3$ as for the spectra of BFP1 and BFP2, respectively (Section 4.2.1). The extended heating model is similar to the spectrum of BFP2, but the NUV exposure time of the BFP2 was adjusted by the IRIS AEC to be $2.4 \mathrm{~s}$. We average over the short and extended 5F11 heating runs in $2.4 \mathrm{~s}$ durations for the first $8 \mathrm{~s}$, which are shown in the top panels of Figure 13; the two emission line components appear in the shorter exposure time average as long as the average is not the first $2.4 \mathrm{~s}$ of the heating.

We calculate the contribution function over the wavelength range of the Fe II $\lambda 2814.45$ line in the 5F11 model. The emergent intensity over the Fe II $\lambda 2814.45$ line and in the NUV (C2826) continuum originate over the same two flaring layers at $z>500 \mathrm{~km}$. The RWA emission component is primarily due to emission from the $\mathrm{CC}$ and the component at $\lambda_{\text {rest }}$ originates primarily from the stationary flare layers just below the CC. In Figure 14, we show the contribution function at $\lambda_{\text {rest }}$ for the 


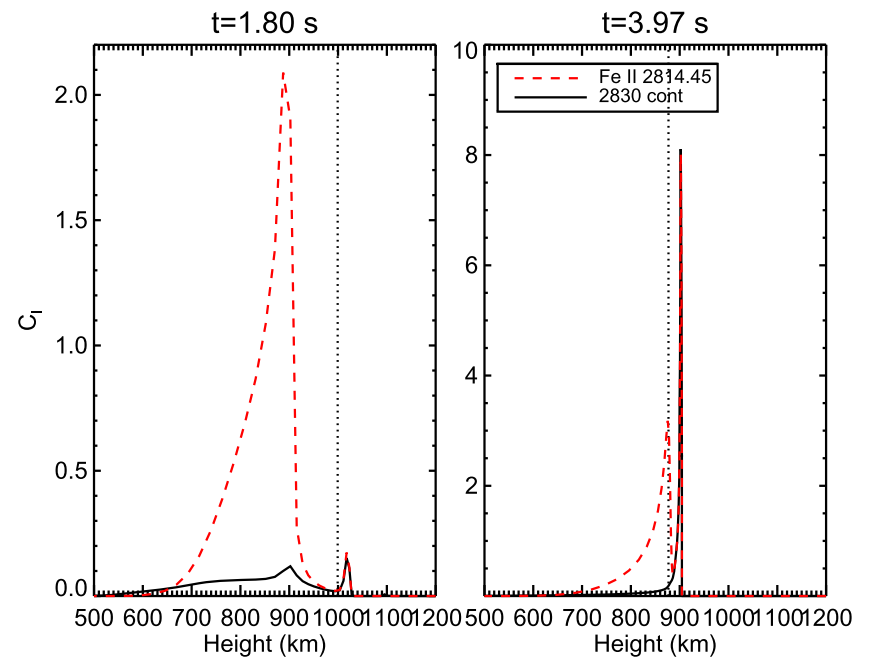

Figure 14. Contribution function for the rest wavelength of Fe II $\lambda 2814.45$ and for $\mathrm{C} 2826$ at $t=1.8 \mathrm{~s}$ and $t=3.97 \mathrm{~s}$ in the $5 \mathrm{~F} 11$ model. The vertical dotted lines indicate the bottom of the chromospheric condensation $\left(z=z_{2}\right.$ in Table 3). A nonthermal broadening parameter of $\xi=7 \mathrm{~km} \mathrm{~s}^{-1}$ is used here and the value of $\tau=1$ for line-center of Fe II $\lambda 2814.45$ is between $z=805-810 \mathrm{~km}$ at both times. Fe II $\lambda 2814.45$ is not completely optically thin and thus probes the conditions in the stationary flare layers from 800 to $900 \mathrm{~km}$; the intensity at $\lambda_{\text {rest }}$ decreases as the chromospheric condensation descends into the stationary flare layers below $z \sim 900 \mathrm{~km}$.

Fe II $\lambda 2814.45$ line $^{14}$ compared to the contribution function for the nearby continuum at $\lambda=2826 \AA$ at $t=1.8 \mathrm{~s}$ and $t=3.97 \mathrm{~s}$. At both times, the optical depth at $\lambda_{\text {rest }}$ reaches a value of $\tau \sim 1$ at $z \sim 800 \mathrm{~km}$ : the Fe II $\lambda 2814.45$ line is not optically thin over the stationary flare layers at $z>500 \mathrm{~km}$. The continuum is optically thin over the heights of Fe II $\lambda 2814.45$ formation and is thus formed over a larger physical depth range. The physical depth ranges are $170 \mathrm{~km}$ (at $1.8 \mathrm{~s}$ ) and $145 \mathrm{~km}$ (at $3.97 \mathrm{~s}$ ) for $\lambda_{\text {rest }}$ of Fe II $\lambda 2814.45$, and the physical depth ranges are $390 \mathrm{~km}$ (at $1.8 \mathrm{~s}$ ) and $160 \mathrm{~km}$ (at $3.97 \mathrm{~s}$ ) for C2826 (using $z_{\lim }=500 \mathrm{~km}$ in Equation (3)).

The relation between the Fe II $\lambda 2814.45$ line profile and the evolution of the flare atmosphere over the first $8 \mathrm{~s}$ of the $5 \mathrm{~F} 11$ heating runs is as follows (see Figure 13 for reference). The $\lambda_{\text {rest }}$ emission component is bright by $0.1 \mathrm{~s}$ due to beam heating in the stationary flare layers at $z>500 \mathrm{~km}$. After $2.5 \mathrm{~s}$, the bright RWA emission component appears at $\lambda-\lambda_{\text {rest }}=35 \mathrm{~km} \mathrm{~s}^{-1}$ as the density of the $\mathrm{CC}$ increases and the temperature decreases to 24,000 K (Figure 9, Table 3). The RWA emission reaches maximum intensity at $4 \mathrm{~s}$ while still centered at $\lambda-\lambda_{\text {rest }}=$ $35 \mathrm{~km} \mathrm{~s}^{-1}$ as the CC decreases to a temperature of $12,000 \mathrm{~K}$; the C2826 intensity also increases in brightness over this time due to increasing density in the CC (Figure 7). For the short 5F11 heating, the continuum drops to the preflare level by $5 \mathrm{~s}$ (the electron beam heating is turned off at $4 \mathrm{~s}$ in the short heating model) and the two emission line components decrease in brightness. Therefore, the average of the first $8 \mathrm{~s}$ of the $5 \mathrm{~F} 11$ with short heating has a relatively brighter $\lambda_{\text {rest }}$ component $\left(I_{\mathrm{RWA}} / I_{\lambda_{\text {rest }}} \ll 1\right)$ because the RWA does not develop until about $t=2.5-3 \mathrm{~s}$. At $t=4-7 \mathrm{~s}$ in the extended 5F11 heating run, the $\lambda_{\text {rest }}$ emission component decreases in intensity while the RWA component remains bright and its peak shifts to

\footnotetext{
${ }^{14}$ We use a constant nonthermal broadening of $\xi=7 \mathrm{~km} \mathrm{~s}^{-1}$ at $z>500 \mathrm{~km}$; see Section 5.6.1.
}

$\lambda-\lambda_{\text {rest }}=15-20 \mathrm{~km} \mathrm{~s}^{-1}$ by $8 \mathrm{~s}$. The $\mathrm{CC}$ maintains a high temperature between 8500 and $11,000 \mathrm{~K}$ with a high density of $4 \times 10^{14} \mathrm{~cm}^{-3}$ over this time as it decreases in speed while moving into the stationary flare layers below. From Table 3, the value of $z_{2}$ (top of the stationary flare layers) has decreased significantly by $7 \mathrm{~s}$ due to the stationary flare layers being swept up by the $\mathrm{CC}$; thus, some material that was emitting $\lambda_{\text {rest }}$ emission at $4 \mathrm{~s}$ emits redshifted emission at $7 \mathrm{~s}$ (similarly, some material that emits $\lambda_{\text {rest }}$ photons at $1.8 \mathrm{~s}$ emits redshifted photons at $3.97 \mathrm{~s}$; see Figure 14).

The broadening and location of the peak of the RWA component in the $8 \mathrm{~s}$ exposure average in the extended 5F11 heating run is due to the velocity evolution of the $\mathrm{CC}$, which decreases from $\sim 45 \mathrm{~km} \mathrm{~s}^{-1}$ at $2 \mathrm{~s}$ to $\sim 18 \mathrm{~km} \mathrm{~s}^{-1}$ at $8 \mathrm{~s}$. The velocity gradient within the $\mathrm{CC}$ at each time also contributes toward emission between $\lambda_{\text {rest }}$ and the peak of the RWA but is relatively minor. Therefore, the average of the first $8 \mathrm{~s}$ of the 5F11 with extended heating has a brighter RWA component $\left(I_{\text {RWA }} / I_{\lambda_{\text {rest }}} \sim 2 / 3\right)$ because of the decreasing intensity of the $\lambda_{\text {rest }}$ emission component from material being swept up by the $\mathrm{CC}$ while the $\mathrm{CC}$ produces bright redshifted emission after $4 \mathrm{~s}$ when the density is increasing and the temperature remains high near $T \sim 10,000 \mathrm{~K}$.

\subsubsection{Nonthermal Broadening}

In the two exposure averages of the 5F11 model in Figure 4 (middle panel), the $\lambda_{\text {rest }}$ emission components in Fe II $\lambda 2814.45$ have comparable peak intensity values (accounting for the different continuum levels), as do the $\lambda_{\text {rest }}$ components in the spectra of BFP1 and BFP2. However, the model $\lambda_{\text {rest }}$ components in the Fe II lines are significantly narrower than the width of this component in the observations (top panel of Figure 4). In these LTE calculations, we include natural damping and thermal broadening, which are small. The quadratic electron pressure broadening is applied using the prescription in the $\mathrm{RH}$ code, but the largest values of the quadratic electron pressure damping parameter in our flare atmospheres are as small as the natural damping parameter and thus do not contribute measurable broadening. It is also known that theoretical quadratic electron pressure broadening using the adiabatic approximation underestimates the damping parameter (Mihalas 1978), but only an extremely large discrepancy by several orders of magnitude could produce the observed broadening. Because the model (black) profile is too narrow in the line-center emission component, we add a nonthermal broadening (microturbulence) parameter, $\xi$.

A nonthermal broadening parameter of $\xi=6-7 \mathrm{~km} \mathrm{~s}^{-1}$ at $z>500 \mathrm{~km}$ is adequate to reproduce the width of the $\lambda_{\text {rest }}$ emission, but the observed broadening of the RWA component is still not well-reproduced. In Figure 4 (middle panel), the RWA component of the model Fe II $\lambda 2814.45$ line is broader than the emission in any instantaneous profile due to the velocity evolution of the $\mathrm{CC}$ over the $8 \mathrm{~s}$ exposure time. The red extent of the model profile is determined by the velocity of the CC when it attains a temperature of $\sim 20,000 \mathrm{~K}$. The extrapolation of the $\mathrm{C} 2826$ continuum intensity to the wavelength range of $\mathrm{Fe}$ II $\lambda 2814.45$ in Figure 4 (top panel) demonstrates that the observed RWA component extends to $\lambda-\lambda_{\text {rest }} \sim 120 \mathrm{~km} \mathrm{~s}^{-1}$ for BFP1; for BFP2, the tail of the RWA component decreases to the continuum level at $\lambda-\lambda_{\text {rest }} \sim 70-80 \mathrm{~km} \mathrm{~s}^{-1}$. 
Table 4

Time-variable Nonthermal Broadening in the $\mathrm{CC}$

\begin{tabular}{lc}
\hline \hline Time $(\mathrm{s})$ & $\xi\left(\mathrm{km} \mathrm{s}^{-1}\right)$ \\
\hline 1 & 68 \\
1.5 & 55 \\
2 & 42 \\
2.5 & 31 \\
3 & 25 \\
4 & 18 \\
5 & 13 \\
6 & 11 \\
7 & 10 \\
8 & 10 \\
15 & 7 \\
\hline
\end{tabular}

Note. Values of the nonthermal broadening (microturbulence) parameter $\xi$ assumed at heights corresponding to the chromospheric condensation; see Section 5.6.1 for details.

A detailed investigation of the physical origin of the nonthermal broadening is outside the scope of this paper; here, we assume the nonthermal broadening in the CC layers is velocity broadening (turbulent broadening with an isotropic Gaussian distribution). To approximate time-dependent velocity broadening in the CC, a simple prescription for $\xi(t)$ is used. We assume that $\xi(t) \propto \rho_{\max }(t)^{-1 / 2}$ where $\rho_{\max }$ is the maximum density of the CC given in Figure 9, and $\xi(t)$ is constant as a function of height throughout the CC. This relation may be reasonable if a constant amount of flare energy goes into generating the kinetic energy of turbulence, such that the turbulent velocity decreases as the CC accretes mass. The evolution of $\xi(t)$ is given in Table 4 and is normalized to $7 \mathrm{~km} \mathrm{~s}^{-1}$ at $15 \mathrm{~s}$, when the RWA emission component merges into the $\lambda_{\text {rest }}$ emission component. A value of $\xi=7 \mathrm{~km} \mathrm{~s}^{-1}$ is kept for the stationary flare layers at $z>500 \mathrm{~km}$.

The LTE, exposure-averaged profile over $8 \mathrm{~s}$ for the $5 \mathrm{~F} 11$ extended heating run with this prescription for $\xi(t)$ is shown as the light blue line in Figure 4 (middle panel) and in the bottom panel of Figure 4 overlayed on the observations. Although the intensity of the model is a factor of $\sim 2$ larger than the observations, the $30 \%$ bisector of $13 \mathrm{~km} \mathrm{~s}^{-1}$ is in satisfactory agreement with the measurement from BFP2 (30\% bisector velocity of $9 \mathrm{~km} \mathrm{~s}^{-1}$; Section 4.2.1). From $t=2$ to $8 \mathrm{~s}$, the velocity of the CC decreases from $\sim 45$ to $\sim 18 \mathrm{~km} \mathrm{~s}^{-1}$; notably, the gas velocity is significantly larger than the $30 \%$ bisector velocity of Fe II $\lambda 2814.45$. The Fe II $\lambda 2814.45$ model profile exhibits a RWA component that extends to redder wavelengths and a profile that is more consistent with the spectrum of BFP2 (top panel) than the calculation without nonthermal broadening (the variable prescription of $\xi(t)$ better accounts for the far red wing emission at $\lambda-\lambda_{\text {rest }} \sim 45-70 \mathrm{~km} \mathrm{~s}^{-1}$ than with a time-independent value of $\xi=7 \mathrm{~km} \mathrm{~s}^{-1}$ in the CC). However, more emission is required to account for the observations in the far red wing at $\lambda-\lambda_{\text {rest }}>70 \mathrm{~km} \mathrm{~s}^{-1}$. In the Mg II $\lambda 2791$ and $k$ lines, the observed RWA components extend to $\lambda-\lambda_{\text {rest }}>100 \mathrm{~km} \mathrm{~s}^{-1}$ and are much brighter (e.g., Figure 3). Although the Fe II $\lambda 2814.45$ line is more optically thin and provides a better diagnostic of the velocity field over the continuum-emitting flare layers, calculations of the Mg II lines with partial frequency redistribution (e.g., Leenaarts et al. 2012; Leenaarts et al. 2013a, 2013b) can be used to better understand the extremely red RWA emission, which we leave to future work.

\subsection{Comparison of Fe II $\lambda 2814.45$ to Fe II $\lambda 2832.39$ LTE Models}

We repeat the LTE calculation for the Fe II $\lambda 2832.39$ line profiles to compare to the observations of BFP1 and BFP2 in Figure 5 (top and middle panels). In the $8 \mathrm{~s}$ average over the extended 5F11 heating model, the peak of the RWA component is comparably bright to the peak of the $\lambda_{\text {rest }}$ emission component $\left(I_{\mathrm{RWA}} / I_{\lambda_{\text {rest }}} \sim 1\right)$ and the peak wavelength is closer to $\lambda_{\text {rest }}$, whereas in the $8 \mathrm{~s}$ average over the short $5 \mathrm{~F} 11$ heating the peak of the RWA emission component is less intense than the peak intensity of the $\lambda_{\text {rest }}$ emission component $\left(I_{\mathrm{RWA}} / I_{\lambda_{\text {rest }}} \sim 1 / 2\right)$ and has a larger redshift. Similar to Fe II $\lambda 2814.45$, the short $5 \mathrm{~F} 11$ heating model (black) spectrum qualitatively resembles the spectrum of BFP1, and the extended 5F11 heating model (pink) spectrum qualitatively resembles the spectrum of BFP2. In the middle and bottom panels of Figure 5, we show the $\mathrm{Fe}$ II $\lambda 2832.39$ profile with the $\xi(t)$ prescription from Section 5.6.1. Although the line intensity is significantly larger than the observations, the value of $I_{\mathrm{RWA}} / I_{\lambda_{\text {rest }}}$ is well-reproduced. Thus, we are able to reproduce the range of observed values of $I_{\mathrm{RWA}} / I_{\lambda_{\text {rest }}}$ in the brightest pixels with the 5F11 model.

The differences in the optical depth between the Fe II $\lambda 2814.45$ and Fe II $\lambda 2832.39$ lines contribute to the differences in the values of $I_{\mathrm{RWA}} / I_{\lambda_{\text {res }}}$ for the exposureaveraged simulated profiles in Figures 4 and 5. The RWA component in the Fe II $\lambda 2832.39$ profile becomes comparably bright to the $\lambda_{\text {rest }}$ component at earlier times $(t=3 \mathrm{~s})$ than for the Fe II $\lambda 2814.45(t=4 \mathrm{~s})$. The time lag to increase to comparable brightness to the emission at $\lambda_{\text {rest }}$ is due to larger optical depth at $\lambda_{\text {rest }}$, and therefore a smaller physical depth range in the stationary flare layers, for Fe II $\lambda 2832.39$. For example, at $3 \mathrm{~s}$ in the $5 \mathrm{~F} 11, \tau_{\lambda \text {,rest }}=1$ at $890 \mathrm{~km}$ for Fe II $\lambda 2832.39$ whereas $\tau_{\lambda \text {,rest }}=1$ at $805 \mathrm{~km}$ for Fe II $\lambda 2814.45$. At $t=3.97 \mathrm{~s}$ in the $5 \mathrm{~F} 11$, the physical depth range ${ }^{15}$ is $145 \mathrm{~km}$ for Fe II $\lambda 2814.45$ at $\lambda_{\text {rest }}$ and is only $30 \mathrm{~km}$ for Fe II $\lambda 2832.39$ at $\lambda_{\text {rest }}$. For Fe II $\lambda 2832.39$, the larger optical depth and smaller physical depth range at $\lambda_{\text {rest }}$ suppresses the $\lambda_{\text {rest }}$ emission component relative to the RWA emission component, producing a $I_{\mathrm{RWA}} / I_{\lambda_{\text {rest }}}>1$ at times also when the beam heating in the stationary flare layers is reduced due to the increasing CC and coronal density (Table 3). The RWA component is brighter than the $\lambda_{\text {rest }}$ emission in Fe II $\lambda 2832.39$ for a longer fraction of the exposure time average, which results in $I_{\mathrm{RWA}} / I_{\lambda_{\text {rest }}} \sim 1$ for the $8 \mathrm{~s}$ exposure averaged profile; for Fe II $\lambda 2814.45$ the value of $I_{\mathrm{RWA}} / I_{\lambda_{\text {rest }}} \sim 2 / 3$ for the $8 \mathrm{~s}$ exposure averaged profile. We speculate that the spectra of Mg II $\lambda 2791.6$ (Figure 3), where the value of $I_{\mathrm{RWA}} / I_{\lambda_{\text {rest }}}$ is larger than for the Fe II $\lambda 2814.45$ line, can also be explained by the larger optical depth at $\lambda_{\text {rest }}$ for this line compared to both of the Fe II lines.

The optical depth in the RWA affects the value of $I_{\mathrm{RWA}} / I_{\lambda_{\text {rest }}}$ in the opposite way: the optical depth of the RWA wavelengths in the Fe II $\lambda 2814.45$ line is always low, but for Fe II $\lambda 2832.39$ the optical depth of the RWA wavelengths is non-negligible and this tends to decrease the value of $I_{\mathrm{RWA}} / I_{\lambda_{\text {rest }}}$. However, the optical depth effects at $\lambda_{\text {rest }}$ contribute the most to the value of $I_{\mathrm{RWA}} / I_{\lambda_{\text {rest }}}$ because the RWA emission originates in the CC,

${ }^{15}$ Using $\xi=7 \mathrm{~km} \mathrm{~s}^{-1}$ at $z>500 \mathrm{~km}$. 
which has a 10x larger density than the stationary flare layers (Table 3), and therefore is very bright even when the physical depth range of emission at RWA wavelengths is comparably small.

\subsection{Comparison of Heating Model Predictions for Fe II}

In Figure 13, we compare the predictions of the 5F11 Fe II $\lambda 2814.45$ spectrum to an LTE calculation for the F11 model (lower left panel) and a coronal heating model (lower right panel) that produces a large conductive flux into the transition region and upper chromosphere. While the 5F11 model satisfactorily explains the two emission line components in Fe II $\lambda 2814.45$, the F11 and coronal heating model do not produce the two line components and the observed excess NUV continuum intensity. An RWA emission component is not produced by the F11 model due to the lack of dense, fast downflows (as discussed in Kuridze et al. (2015), for this model), and there is only bright emission at $\lambda_{\text {rest }}$. The coronal heating model produces a relatively bright RWA component in Fe II $\lambda 2814.45$ and a $30 \%$ bisector velocity of $30 \mathrm{~km} \mathrm{~s}^{-1}$ for $\mathrm{H} \alpha$ (Table 2), but the excess NUV continuum emission in the coronal heating model is faint $\left(\leqslant 4 \times 10^{5} \mathrm{erg} \mathrm{cm}^{-2} \mathrm{~s}^{-1} \mathrm{~s}\right.$. $\mathrm{r}^{-1} \AA^{-1}$ ) and is a factor of 6 below the observed values in BFP1 and BFP2 (Table 1). In the coronal heating model, high velocity, Balmer continuum emitting layers are produced as in the 5F11 model but with much lower emergent continuum intensity. The stationary flare layers at $z \sim 750 \mathrm{~km}$ are heated to $T \sim 7000 \mathrm{~K}$ (from $T \sim 4460 \mathrm{~K}$ at $t=0 \mathrm{~s}$ ) and produce the Fe II emission at $\lambda_{\text {rest }}$. The temperature increase in the stationary flare layers is due to backwarming from the absorption of NUV photons in the range from $\lambda \approx$ 1500-3000 $\AA^{16}{ }^{16}$ In the coronal heating model, the RWA for the $\mathrm{Fe}$ II $\lambda 2814.45$ line develops before the line center emission, whereas the $5 \mathrm{~F} 11$ beam model exhibits a $\sim 2.5 \mathrm{~s}$ delay in the brightening of the RWA after the initial brightening of the line-center component. High time-resolution $(\Delta t<1 \mathrm{~s})$ spectra and hard X-ray data could be useful to distinguish between these two heating scenarios (Canfield \& Gayley 1987).

\subsection{The RWA Component in Chromospheric Emission Lines}

We hypothesize that the spectrally resolved redshifted components (RWA) in the Fe II line profiles of the 5F11 model are analogous to the RWA often observed in the $\mathrm{H} \alpha$ line profile which has been attributed to the phenomenon of chromospheric condensation (Ichimoto \& Kurokawa 1984; Canfield \& Gayley 1987; Canfield et al. 1990). In future work, we will improve on the modeling predictions of the $\mathrm{H} \alpha$ line in Figure 8. To accurately model the $\mathrm{H} \alpha$ line, we will include an improved prescription of the electron pressure broadening and explore the effect of the large microturbulence parameter inferred from the IRIS lines (the standard value of $\xi$ used in RADYN is $2 \mathrm{~km} \mathrm{~s}^{-1}$ ). In the $5 \mathrm{~F} 11$ model, this line is very optically thick and does not have a significant emission component at $\lambda_{\text {rest }}$ that originates from the stationary flare layers; the entire line exhibits a redshift at $t=3.97 \mathrm{~s}$ (Figure 8), which is qualitatively consistent with some previous flare observations of $\mathrm{H} \alpha$ (Canfield et al. 1990) but not the

\footnotetext{
${ }^{16}$ This type of chromospheric heating will be discussed in detail in a future modeling study of stellar flare data from the Hubble Space Telescope (Kowalski et al. 2017, in preparation).
}

observations of Ichimoto \& Kurokawa (1984). However, when a redshift of the entire line is observed during flares, the maximum redshift occurs when the intensity of the line is low and this is not predicted by the 5F11 model.

The wavelength range of the observations of $\mathrm{H} \alpha$ for this flare (Kleint et al. 2015; Rubio da Costa et al. 2016) from the DST/ IBIS (Cavallini 2006) is indicated in Figure 8. The 5F11 prediction at $t=1.8 \mathrm{~s}$ predicts that $I_{6564} \approx I_{6563}>I_{6562}$ as in the observations of the brightest regions of the flare (cf. Figure 2 panel $b$ of Rubio da Costa et al. 2016), but the intensity of the model is a factor of 5-10 larger than these observations.

\section{The Brightest SJI 2832 Kernel in the 2014 March 29th X1 Flare}

The excess $\mathrm{C} 2826$ in the $5 \mathrm{~F} 11$ model reaches a maximum of $5-5.5 \times 10^{6} \mathrm{erg} \mathrm{cm}^{-2} \mathrm{~s}^{-1}$ s.r. ${ }^{-1} \AA^{-1}$ between $t=4$ and $t=6 \mathrm{~s}$ (Figure 7), which is significantly larger than the highest values of excess C2826 obtained from the spectra of BFP1 and BFP2 $\left(\sim 2-2.2 \times 10^{6} \mathrm{erg} \mathrm{cm}^{-2} \mathrm{~s}^{-1}\right.$ s.r. $\left.^{-1} \mathrm{~A}^{-1}\right)$. The Fe II emission line profiles for the extended heating run (those that produce the strongest RWA components) also produce very bright emission line profiles that are a factor of 1.5-3 larger than the observations (bottom panels of Figures 4 and 5), while the short heating prediction for the Fe II $\lambda 2814.45$ intensity is rather well-reproduced (middle panel of Figure 4). However, the locations of BFP1 and BFP2 are not the brightest locations during the impulsive phase due to the long rastering cadence of $\sim 75 \mathrm{~s}$ and the relatively small spatial coverage of the slit, which notably misses the brightest regions of the $\left(\sim 10^{16} \mathrm{~cm}^{2}\right)$ flare kernel BK2830 at $(x, y)=(519.5,263 . " 8)$ in SJI 2832 \#173 (Figure 1). BK2830 corresponds to the location of the hard X-ray source revealed by RHESSI imaging of the plage ribbon (Kleint et al. 2016). As noted by Kleint et al. (2016), the IRIS raster intersects the half-maximum of this hard X-ray source.

Using the intensity calibration of SJI 2832 (Section 2), we estimate the maximum excess continuum intensity in BK2830 to be $\sim 10^{7} \mathrm{erg} \mathrm{cm}^{-2} \mathrm{~s}^{-1}$ s.r. $^{-1} \AA^{-1}$ and $\sim 0.7 \times 10^{7} \mathrm{erg} \mathrm{cm}^{-2} \mathrm{~s}^{-1} \mathrm{~s}$. r. $^{-1} \AA^{-1}$ in SJI 2832 \#173 (mid peak phase) and \#174 (early fast decay phase), respectively. These values are 3-4.5 times larger than the values of excess $\mathrm{C} 2826$ obtained from the spectra and are a factor of $\sim 10$ larger than the preflare values. The excess SJI 2832 emission includes several Fe II emission lines, which may exhibit bright RWA components. Unfortunately, the spectral region corresponding to the SJI 2832 wavelengths was not readout for these observations, and we cannot determine the relative contributions for this flare. Using full spectral readout data during an X-class flare on 2014 Oct 25 (Kowalski et al. 2016, in preparation), we identify that the bright Fe II emission lines within SJI 2832 wavelength range include Fe II $\lambda 2826.58$, $\lambda 2826.85, \lambda 2828.26, \lambda 2829.46, \lambda 2829.51$ and $\lambda 2832.39$, and these account for a significant fraction of the SJI 2832 bandpass intensity.

To estimate the SJI 2832 intensity predicted by the $5 \mathrm{~F} 11$ model, we use the LTE approximation for these Fe II lines at $t=3.97 \mathrm{~s}$ as done for the $\mathrm{Fe}$ II $\lambda 2814.45$ and $\mathrm{Fe}$ II $\lambda 2832.39$ lines in Section 4.2.1 and include the continuum opacity and emissivity. The intensity spectrum is converted to $\mathrm{DN} \mathrm{s}^{-1}$ pix $^{-1}$ by integrating over the effective area curve of SJI 2832, which is then converted to a flux density as done for the data (Section 2). The excess SJI 2832 intensity from the 5F11 model at $t=3.97 \mathrm{~s}$ corresponds to $\sim 10^{7} \mathrm{erg} \mathrm{cm}^{-2} \mathrm{~s}^{-1}$ s.r. $^{-1} \AA^{-1}$, 
which is a lower bound because a few emission lines in the bandpass aside from Fe II are not included in the calculation. At its brightest times, the 5F11 model is consistent with the excess intensity from the brightest pixels of BK2830 in SJI 2832 image \#173. Furthermore, this model predicts that the excess SJI 2832 intensity values include nearly equal contributions from flare continuum emission and Fe II lines. In Section 3, we inferred similar proportions of line and continua emission using spatially coincident but temporally contiguous observations from the slit jaw images and spectra.

\section{Model Uncertainties and Assumptions}

In our modeling approach, we have made several assumptions that warrant discussion in light of our findings.

\subsection{The LTE Assumption for Fe II $\lambda 2814.45$ and Fe II $\lambda 2832.39$ Profiles}

Using the RH code for the $5 \mathrm{~F} 11$ at $t=3.97 \mathrm{~s}$ and a large Fe II atom (Walkowicz et al. 2008) that comes with the $\mathrm{RH}$ distribution, we confirmed that the LTE profile of Fe II $\lambda 2814.45$ satisfactorily matches the NLTE profile from RH. All relevant sources of broadening (including electron pressure broadening) are included in this calculation, which justifies implementing an ad hoc microturbulent parameter to match the line broadening. Because the LTE assumption is employed at all time steps in the simulations to produce the exposure-averaged spectra, the spectra for the Fe II $\lambda 2814.45$ and Fe II $\lambda 2832.39$ lines (Section 5.6) are approximations. The LTE modeling assumption of the Fe II lines within the SJI 2832 camera effective area at $t=3.97 \mathrm{~s}$ provides a reasonable approximation to the amount of excess SJI 2832 emission that is due to Fe II flare lines with bright RWA components because the collisional rates are high at this time.

\subsection{Return Current and Beam Instability Effects}

The large electron flux $\left(8.5 \times 10^{18}\right.$ electrons $\left.\mathrm{s}^{-1} \mathrm{~cm}^{-2}\right)$ for the 5F11 beam model produces an electric field given that nonthermal protons do not neutralize the beam (Emslie 1980; van den Oord 1990). Using the equations from Holman (2012), we estimate that an energy loss of $\sim 30 \mathrm{keV}$ per beam electron would occur over the upper $8 \mathrm{Mm}$ of the corona due to the return current electric field (at $t=0 \mathrm{~s}$ ). The return current electric field imparts a drift velocity to ambient electrons, and the energy loss from the beam over the potential drop thus increases the thermal energy of the corona from the Joule heating (Holman 1985, 2012).

We have used an approximate prescription for the heating from the return current $\left(Q_{\mathrm{RC}}\right)$ in the F11 and 5F11 models (Holman 2012; Allred et al. 2015). The maximum temperature in the corona for the $5 \mathrm{~F} 11$ model at $4 \mathrm{~s}$ is $30 \mathrm{MK}$ and is due to comparable amounts of return current heating and beam heating; after $10 \mathrm{~s}$, the maximum temperature exceeds 50 MK. We note that a coronal temperature of $T \sim 30 \mathrm{MK}$ is reasonable compared to the RHESSI observations ( $T \sim 25 \mathrm{MK}$; Battaglia et al. 2015) but a detailed emission measure analysis of the model Fe XXI line in the IRIS range is necessary for a meaningful comparison. The mean free path of the ambient drifting electrons (at $t=0 \mathrm{~s}$ ) is $\sim 150 \mathrm{~km}$ so they are thermalized in a relatively short distance. At all times, we assume the electrons are thermalized over a short distance so that the return current is in steady state $\left(E_{\mathrm{RC}}=\eta J_{\text {beam }}\right.$, where $\eta$ is the plasma resistivity). We also assume that ambient electrons are not accelerated out of the thermal distribution, but the $5 \mathrm{~F} 11$ is at the threshold where this assumption starts to break down.

High beam fluxes of $\gtrsim 10^{12} \mathrm{erg} \mathrm{cm}^{-2} \mathrm{~s}^{-1}$ are commonly inferred from high spatial resolution flare data of the brightest flaring footpoints for small and large GOES class flares alike (L. Fletcher 2015, private communication; Hudson et al. 2006; Fletcher et al. 2007; Krucker et al. 2011; Gritsyk \& Somov 2014; Milligan et al. 2014; Jing et al. 2016) and return currents have been attributed to the spectral differences between coronal and chromospheric hard X-ray sources (Battaglia \& Benz 2006, 2008). A method for determining the importance of return currents in a large sample of flares (including the SOL2014-03-29T17:48 flare) using a self-consistent analysis of the soft and hard X-ray spectra will be presented in Alaoui et al. 2017 , in preparation. The potential drop from a return current electric field is expected to flatten the electron distribution function at low energies (Zharkova \& Gordovskyy 2006), but the early impulsive phase of the SOL2014-03-29T17:48 flare can be fit satisfactorily by a single power law (Kleint et al. 2016; Alaoui et al. 2017, in preparation). The presence of a large potential drop of $30 \mathrm{keV}$ from a high flux (5F11) beam at $t=0 \mathrm{~s}$ cannot be affirmed from the hard X-ray data of this flare, but the potential drop is expected to lower as the resistivity decreases as the corona heats up from its initial temperature.

The 5F11 beam density is $10 \%$ of the ambient coronal (preflare) density, which is $8 \times 10^{9} \mathrm{~cm}^{-3}$ in our model and is consistent with the upper limits of the solar coronal density above an active region (Krucker et al. 2010). This beam density results in a return current drift speed of $\sim 10^{9} \mathrm{~cm} \mathrm{~s}^{-1}$ and exceeds the electron thermal speed of the pre-flare corona. We do not consider the effects of beam instabilities and doublelayers that result from a large return current drift speed (e.g., Lee et al. 2008; Li et al. 2014) or from a sharp low-energy cutoff (Hannah et al. 2009). The effects from beam instabilities (which can form on very short timescales) for $n_{\text {beam }} / n_{\text {bckgd }}<1$ will likely need to be addressed in more detail in future work on high beam fluxes.

Lower electron beam fluxes have been considered in previous radiative hydrodynamic flare modeling work of this flare, but these simulations do not produce the bright red wing emission of the $\mathrm{Mg}$ II $h$ and $k$ lines although the $\lambda_{\text {rest }}$ intensity is closer to the observations (Rubio da Costa et al. 2016). The F11 model also produces a continuum intensity that is inadequate to explain the brightest footpoint emission (see also Heinzel et al. 2016). A combination of several heating mechanisms, such as electron beams, proton/ion beams (Allred et al. 2015), and Alfvén waves (Russell \& Fletcher 2013; Reep \& Russell 2016) may alleviate the requirement of large nonthermal electron beam fluxes to account for both the dense, heated CCs and stationary flare layers in the impulsive phase. However, one must still account for the observed hard X-ray bremsstrahlung emission. Rubio da Costa et al. (2015) presented a novel modeling method using an initial electron distribution from stochastic acceleration theory (Petrosian \& Liu 2004), which could produce downflows for low beam fluxes thus mitigating return current effects (as also suggested by Rubio da Costa et al. 2016, for this flare). The downflows are conductively driven from low-energy electron energy deposition in the corona. It remains to be determined whether 
beam distributions from stochastic acceleration theory have enough energy in high energy electrons to heat the downflows and stationary flare layers enough to produce the observed NUV continuum intensity. Alternatively, it has been proposed that the bulk of the nonthermal electrons are accelerated by Alfvén wave energy transported to lower heights in the atmosphere (Fletcher \& Hudson 2008).

\subsection{Spatial Resolution}

We compare the $(\mu=0.77)$ intensity from the model to the observations, which requires that the NUV continuum footpoints are sufficiently spatially resolved. The spatial profiles of BFP1 and BFP2 have a FWHM of $\sim 11$ pixels (Section 3, Figure 6) and thus they are resolved. We estimated the leading edge spatial extent to $\sim 7$ pixels, which is also adequately resolved. Several locations of the ribbon, such as the third bright footpoint BFP3 $\left((x, y) \sim\left(520^{\prime \prime}, 262^{\prime \prime}\right)\right.$ in Figure 1$)$, do not appear resolved. If the filling factor of an IRIS resolution element is significantly less than 1 , then the model continuum intensity must be degraded and a series of sequentially heated elements would be more comparable to the observations from a single IRIS resolution element (Heinzel et al. 2016).

\subsection{Other Modeling Approximations}

Ionization equilibrium is assumed for atoms and ions included in the optically thin loss function. Also, several ions (Fe II, Si II, Mg II) are excluded from the optically thin loss function because these elements are likely optically thick at low temperature. The ionization fraction of neutral magnesium is not included in the calculations of the $\mathrm{Mg}$ II wing opacities. The Mg I 2852 line wing affects the intensity in the wavelength range of SJI 2832 (Leenaarts et al. 2013b), but this is likely a small effect compared to the chromospheric flare intensity produced in the 5F11 model. Mg I also has edges in the NUV (e.g., $\lambda=2513 \AA$ ), and the population may be reduced in the lower atmosphere during the flare from absorption of Balmer continuum photons. We employ a 3 level+continuum $\mathrm{Mg}$ II ion for the RH calculations, and using a more complex model ion (like that in Leenaarts et al. (2013a); Rubio da Costa et al. (2016)) may slightly affect the wing emission in the upper photosphere.

\subsection{Future Work}

Solar flares exhibit a range of inferred electron beam parameters (e.g., Fletcher et al. 2007; Kuhar et al. 2016), properties of the Balmer jump spectral region (e.g., Neidig 1983), H $\alpha$ profiles (e.g., Ichimoto \& Kurokawa 1984; Canfield et al. 1990; Kuridze et al. 2015), and $\mathrm{Mg}$ II $h$ and $k$ profiles (e.g., Graham \& Cauzzi 2015; Kerr et al. 2015). A comprehensive flare model must be able to self-consistently explain the range of these properties observed in different flares. In future work, we will analyze IRIS observations of the brightest kernels in many flares and use the NUV continuum and Fe II modeling techniques developed here to compare to RHD simulations covering a range of electron beam parameters inferred from RHESSI. We will also employ constraints from the FUV continuum from IRIS for a multiwavelength characterization of the continuum distribution. Higher cadence sit-and-stare observations will be used to improve on the constraints of time-evolution of the continuum intensity and RWA evolution (Section 5.6).
In future modeling work for the March 29th flare, we will explore high-flux ( $\geqslant \mathrm{F} 11)$ beam models with $E_{c}<25 \mathrm{keV}$ to determine if these models produce a more accurate exposureaveraged continuum intensity and Fe II line profile shapes and intensity; the $5 \mathrm{~F} 11$ with $E_{c}=25 \mathrm{keV}$ model achieves a continuum and line brightness that exceeds the spectroscopic constraints by a factor of $\sim 2$. We will also incorporate the NLTE predictions of the Mg II line profiles (as in Rubio da Costa et al. (2016)) with the effects of nonthermal collision rates and compare our CC models in detail to observations of $\mathrm{H} \alpha$ with the DST/IBIS (Kleint et al. 2015; Rubio da Costa et al. 2016) and to lines from IRIS that probe hotter temperatures (Young et al. 2015). In flares, the Si IV lines also exhibit two, spectrally resolved emission components (referred to as "CB" and "CR" Brannon et al. 2015) which will be compared to the $\lambda_{\text {rest }}$ and RWA components in the chromosphere flare lines.

Beam propagation effects such as the return current will need to be addressed in future work modeling of the impulsive phase. The self-consistent treatment of energy loss from the beam and ambient heating will be included in the FokkerPlanck equation and modeled in future work (Allred 2017, in preparation). A prescription for ramping the beam flux down (Kašparová et al. 2009) and other gradual phase heating mechanisms will be necessary for a comparison to observations of the gradual phase. An abrupt turn-off of the flux in the short heating $5 \mathrm{~F} 11$ run results in a $\sim 100 \%$ decrease in the NUV continuum within two seconds, whereas the observations show that continuum intensity decreases by only $\sim 80 \%-90 \%$ in $75 \mathrm{~s}$ (Figure 3).

\section{Summary}

We modeled the $1 \mathrm{D}$ radiative-hydrodynamic response of the solar atmosphere to a high energy flux density of nonthermal electrons $\left(5 \times 10^{11} \mathrm{erg} \mathrm{cm}^{-2} \mathrm{~s}^{-1}\right)$ similar to that inferred from the thick target modeling of RHESSI hard X-ray observations combined with high spatial resolution areal constraints (Kleint et al. 2016). The model comparison to the data is summarized in Table 5. We found that the NUV continuum intensity and Fe II line profile shapes in the 5F11 model are in satisfactory agreement with the observations of the brightest flare footpoints that were observed spectroscopically during the SOL2014-03-29T17:48 X1 solar flare. Given the uncertainty in the duration and time-profile of the beam heating, we used a constant heating rate for a short time (4s) and an extended time $(15 \mathrm{~s})$. The instantaneous excess continuum intensity near $\lambda \sim 2826 \AA$ in the 5F11 is achieved after only a few seconds of beam heating, and an exposure average including the early rise phase emission and the development of the chromospheric condensation is necessary to reproduce the two chromospheric $\mathrm{Fe}$ II emission line profile components. The Fe II chromospheric emission lines and NUV continuum intensity originate over two flare layers and are complementary constraints on the model predictions of flare heating and the atmospheric conditions that produce white-light emission.

\section{Conclusions}

The conclusions from our work are the following:

1. The radiative-hydrodynamic modeling of the atmospheric response to a high flux (5F11) nonthermal electron beam is an improvement over static flare modeling because the 
Table 5

Model Comparison Checklist and Where They Are Not Compared with the Data in This Work ( $\cdots)$

\begin{tabular}{|c|c|c|c|c|c|}
\hline Time & $\begin{array}{l}\text { NUV Continuum Intensity } \\
\text { Excess C2826 }\end{array}$ & $\begin{array}{c}\lambda_{\text {rest }} \text { Intensity } \\
\text { Fe II } \lambda 2814.45, \\
\text { Fe II } \lambda 2832.39\end{array}$ & $\begin{array}{c}I_{\mathrm{RWA}} / I_{\lambda_{\text {rest }}} \\
\text { Fe II } \lambda 2814.45, \\
\text { Fe II } \lambda 2832.39\end{array}$ & $\begin{array}{l}\mathrm{H} \alpha \text { Bisector } \\
\geqslant 30 \mathrm{~km} \mathrm{~s}^{-1}\end{array}$ & SJI 2832 Intensity \\
\hline
\end{tabular}

\begin{tabular}{|c|c|c|c|c|c|}
\hline \multicolumn{6}{|l|}{$5 \mathrm{~F} 11$} \\
\hline$t=1.8 \mathrm{~s}$ & $\checkmark$ & $>, \cdots$ & $\mathrm{X}, \cdots$ & $<$ & $\cdots$ \\
\hline$t=3.97 \mathrm{~s}$ & $>$ & $>, \cdots$ & $\checkmark, \cdots$ & $\checkmark$ & $\checkmark$ \\
\hline$t=0-8 \mathrm{~s}$ ave (short heating) & $<$ & $\checkmark,>$ & $\checkmark, \checkmark$ & $\cdots$ & $\cdots$ \\
\hline $\begin{array}{l}t=0-8 \mathrm{~s} \text { ave (extended } \\
\text { heating) }\end{array}$ & $>$ & $>,>$ & $\checkmark, \checkmark$ & $\cdots$ & $\cdots$ \\
\hline \multicolumn{6}{|l|}{ F11 } \\
\hline$t=3 \mathrm{~s}$ & $<$ & $>, \cdots$ & $\mathrm{X}, \cdots$ & $\mathrm{X}$ & $\cdots$ \\
\hline$t=18 \mathrm{~s}$ & $<$ & $>, \cdots$ & $\mathrm{X}, \cdots$ & $\mathrm{X}$ & $\cdots$ \\
\hline \multicolumn{6}{|l|}{ Coronal heating model } \\
\hline$t=3 \mathrm{~s}$ & $<$ & $<, \cdots$ & $>, \cdots$ & $\checkmark$ & $\ldots$ \\
\hline
\end{tabular}

Note. For each of the models at selected times, we indicate where they are consistent with the data constraints $(\checkmark)$, where they exceed the data constraints $(>)$, where they are too low $(<)$, where they fail altogether to produce the indicated feature ("X"), and where they are not compared to the data in this work (-). Except for the last column, the data that we compare to are the quantities obtained from BFP1 at 17:46:08 and BFP2 at 17:46:24 (Table 1), and the H $\alpha$ bisector is used as a proxy for the bisector of an optically thick chromospheric line.

dynamic effects on the emergent spectrum can be critically examined and compared to redshifted emission line components. The NUV continuum brightness changes due to the atmosphere density evolution within a heated, chromospheric compression (condensation) that develops on short (several second) timescales in the 5F11 model. The 5F11 produces an electron density $\left(\sim 4-5 \times 10^{13} \mathrm{~cm}^{-3}\right)$ in the early phase that is consistent with the values inferred from previous static, slab modeling of the Balmer continuum and lines (DonatiFalchi et al. 1985), but the density in NUV continuum emitting layers increases by another order of magnitude as the condensation develops.

2. In the brightest spectra of the SOL2014-03-29T17:48, the excess NUV continuum excess can be explained by hydrogen Balmer recombination emission over several hundred $\mathrm{km}$ at chromospheric heights $(z \sim 630-1020 \mathrm{~km})$ with low optical depth $\left(\tau_{2826} \leqslant 0.2\right)$. The excess NUV continuum emission originates over two flaring layers that are heated by the nonthermal electron beam: a chromospheric condensation with vertical downward velocities of $\sim 20-55 \mathrm{~km} \mathrm{~s}^{-1}$ and stationary flare layers just below the condensation.

A variety of methods have inferred a low optical depth over the region producing the white-light continuum intensity (Hudson 1972; Neidig 1983; Potts et al. 2010; Heinzel \& Kleint 2014). The high flux 5F11 RHD model demonstrates that a heated, downflowing compression increases the density to large values $\left(n_{\mathrm{H} \text {,max }} \sim 5 \times 10^{14}\right.$ $\mathrm{cm}^{-3}$ ) after $4 \mathrm{~s}$ when the continuum has nearly reached maximum brightness, but this density is not enough to produce a large optical depth.

3. The low energy electrons $(E \sim 25-50 \mathrm{keV})$ in the beam heat the chromospheric condensation and higher energy electrons $(E \gtrsim 50 \mathrm{keV}$ initially, $E \gtrsim 80 \mathrm{keV}$ after the condensation becomes dense) heat the stationary flare layers. In the first few seconds of the beam heating the
NUV continuum emission in the stationary flare layers contributes to the majority of the emergent intensity enhancement. Therefore, we expect less continuum intensity in the first few seconds of footpoint brightening (before the $\mathrm{CC}$ becomes bright and dominates the contribution to the emergent intensity) for flares with softer (higher $\delta$ ) time-averaged electron beams (but for same energy flux) because fewer $E>50 \mathrm{keV}$ electrons are available to heat the stationary flare layers. Although there has been no significant observational relationship established between hard X-ray spectral hardness and flare peak optical continuum intensity (Matthews et al. 2003; Kuhar et al. 2016), higher cadence observations than currently available for flare white-light emission are needed to critically test the models by constraining the properties of the continuum emission when it predominantly originates from the stationary flare layers, before the RWA line components develop. It has been proposed that the hardness of the electron beam for very high beam fluxes could explain the interflare variation in observed continuum flux ratios in dMe flares (Kowalski et al. 2016).

4. We have developed a technique to include the $\mathrm{Mg}$ II $h+k$ wing opacity in the calculations of the excess continuum intensity for an accurate comparison to IRIS NUV observations. The far wing opacities are important for an accurate treatment of (sub-)photospheric continuum dimming as well as the amount of $\mathrm{Mg}$ II wing emission in the upper photosphere in response to a moderate temperature change. If these opacities are neglected, errors of $15 \%-30 \%$ result for the excess continuum intensity in lower beam flux models such as the F11 model (Table 2).

5. The Fe II $\lambda 2814.45$ and Fe II $\lambda 2832.39$ profiles provide new information on the dynamics in continuum-emitting layers at $T \sim 8500-25,000 \mathrm{~K}$ and electron densities of $\sim 5 \times 10^{13}-5 \times 10^{14}$. The LTE Fe II line profiles 
predicted by the 5F11 are qualitatively consistent with the spectral observations of the brightest flaring footpoints: the profiles are spectrally resolved and exhibit an emission component close to the rest wavelength that is produced in the stationary flare layers and a bright redshifted emission component that is produced in the chromospheric condensation.

6. The physical depth range parameter is an important parameter for understanding how the emergent intensity varies as a function of wavelength for emission lines and continua because the physical depth range reflects the variation of $\tau_{\lambda}$. The Fe II $\lambda 2814.45$ line is optically thin enough at line center to probe the conditions over a significant physical depth range in the flare chromosphere, but is more optically thick than the NUV continuum intensity. Interestingly, the spectrum of BFP1 (with an exposure time of $8 \mathrm{~s}$ ) and the spectrum of BFP2 (with an exposure time of $2.4 \mathrm{~s}$ ) exhibited a similar excess NUV continuum intensity and brightness in the rest-wavelength component of the chromospheric emission lines, but showed striking differences in the strength and redshift of the RWA emission component in the chromospheric line profiles (Figures 4 and 5).

The range of relative brightnesses of the two emission components $\left(I_{\mathrm{RWA}} / I_{\lambda_{\text {rest }}}\right)$ for each Fe II line is adequately reproduced in the 5F11 model (using simulated exposure times of $2.4-8 \mathrm{~s}$ ). The differences in $I_{\mathrm{RWA}} / I_{\lambda_{\text {rest }}}$ between Fe II $\lambda 2814.45$ and Fe II $\lambda 2832.39$ are due to optical depth differences at $\lambda_{\text {rest }}$ : Fe II $\lambda 2832.39$ is more optically thick at $\lambda_{\text {rest }}$ and has a larger value of $I_{\mathrm{RWA}} / I_{\lambda_{\text {rest }}}$. Although there may be departures from LTE that are not accounted for in our models of Fe II, the LTE assumption identifies several important atmospheric parameters that lead to the line profile properties over an exposure time: (1) the brightness at $\lambda_{\text {rest }}$ is determined by the optical depth at line center, the beam energy deposition evolution, and the extent to which the stationary flare layers have been accrued by the chromospheric condensation; (2) the peak wavelength (and to some extent the broadening) of the redshifted emission line component is determined by the density and velocity evolution of the $\mathrm{CC}$ as it cools from $T=25,000 \mathrm{~K}$ to $T=8500 \mathrm{~K}$.

7. The Fe II lines do not exhibit measurable thermal or pressure broadening, and a nonthermal broadening is required to account for the width of the observed line component at $\lambda_{\text {rest }}$. We find that a time-variable nonthermal broadening given by a simple physical prescription for velocity broadening is appropriate for the profile shape, reproduces the observed bisector velocity, and accounts for some of the far red emission of the RWA emission component.

8. The coronal heating model and 5F11 beam model produce redshifts of the $\mathrm{H} \alpha$ line by $\sim 30 \mathrm{~km} \mathrm{~s}^{-1}$ and a relatively bright RWA emission component in the LTE model of the Fe II $\lambda 2814.45$ line, but the conductive heating flux into the chromosphere does not produce bright NUV continuum intensity as observed in the spectra of BFP1 and BFP2.

9. At the brightest times in the 5F11, the continuum intensity exceeds the spectroscopic constraints of the continuum intensity, and the predicted Fe II profiles are very bright (a factor of 1.5-3 brighter than the spectral observations). The goal of this study is not to precisely match all possible observables to the model intensity values, given the limits on spatial resolution and our simplified prescription of the electron beam heating function such as a constant heating flux and not including return current effects. Our goal is to quantify the range of continuum brightness values and line profile shapes achieved by the models and determine if they sweep through the regime of the observations as we change the beam flux to the highest values that are within reason. In future work, we intend to explore the range of fluxes between F11 and 5F11, which were chosen to bracket the value of the inferred flux for the brightest kernel (Kleint et al. 2016), and the range of possible values of $E_{c}$. A $2.5 \mathrm{~F} 11$ - 3.5F11 may produce a closer match to the observed intensity in the spectra. We speculate that a softhard-soft variation as observed in short hard X-ray flare bursts on the Sun (e.g., Fletcher \& Hudson 2002; Grigis $\&$ Benz 2004) may also help reduce the rest-wavelength intensity averaged over an exposure time because less beam energy is deposited in the stationary flare layers for softer (higher $\delta$ ) beams.

Nonetheless, we have tested the 5F11 against the slit jaw constraints of the brightest kernel in the flare and found that the very bright continuum and Fe II emission lines at $t \sim 4 \mathrm{~s}$ are consistent with these constraints (Section 6). However, spectroscopic confirmation of these extremely bright continuum and emission line intensities is necessary. In a follow-up paper on the 5F11 model, we present optical predictions of the Balmer and Paschen jump region at $t=3.97 \mathrm{~s}$ using the modeling techniques of the Balmer edge region in $\mathrm{dMe}$ flares (Kowalski et al. 2015b) in order to test the 5F11 model against future spectral observations of the brightest flaring kernels, such as with the Daniel K. Inouye Solar Telescope.

A high flux electron beam using the free-streaming, thicktarget model reproduces several of the critical observations for the March 29th 2014 X1 flare, but the high nonthermal electron flux of 5F11 will require further modeling of the energy loss from the return current electric field as well as including the effects of beam instabilities. Despite the simplifications in the thick target electron beam model employed here, we conclude that flare heating with a high flux electron beam can be used as a powerful tool to interpret spectral phenomena and to understand important radiative-hydrodynamic processes in the brightest flaring footpoints. The consistencies between the 5F11 model predictions and the IRIS observations will serve as a benchmark for models with additional physical processes that address observational challenges (Battaglia \& Benz 2006; Krucker et al. 2011; Martínez Oliveros et al. 2012; Dickson \& Kontar 2013; Simões \& Kontar 2013) to the standard electron beam model for the brightest hard X-ray flare footpoints. Measurements of the Balmer jump ratio as a constraint on optical depth, the broadening of the hydrogen lines as a constraint on electron density, and modeling lines such as Si I (Judge et al. 2014) for constraints on the heating of the upper photospheric layers will complement future comparisons of IRIS flare spectra and RHD models.

We thank an anonymous referee for improvements to the manuscript. A.F.K. thanks Dr. L. Kleint for helpful discussions about the 2014 March 29 flare, Dr. J. Klimchuk for discussions 
on non-thermal broadening and coronal heating, Dr. F. Reale for the suggestion to compare to a coronal heating model, Dr. A. Inglis and Dr. B. Dennis for discussions about RHESSI imaging and spectroscopy, Dr. S. Jaeggli for discussions on IRIS data, Dr. J. Drake for discussions about beam instabilities and double-layers, Dr. H. Uitenbroek for the use of the RH code, M. Alaoui for helpful discussions on the return current effects during solar flares, Dr. G. Del Zanna for helpful conversations about non-equilibrium ionization, and Dr. $\mathrm{H}$. Hudson, Dr. L. Fletcher for helpful discussions about this work. AFK also acknowledges helpful discussions at Dr. P. Testa's workshop on microflare heating at the International Space Science Institute and discussions at Dr. L. Harra's workshop on energy transformation in solar and stellar flares at the International Space Science Institute, AFK acknowledges funding that supported this work from the NASA Heliophysics Guest Investigator Grant NNX15AF49G and funding from the Goddard Planetary Heliophysics Institute (GPHI) Task 132, and support from the AAS Solar Physics Division 2015 Metcalf Travel Award to present results from this work at the Hinode 9 workshop at Queen's University Belfast. The research leading to these results has received funding from the European Community's Seventh Framework Programme (FP7/2007-2013) under grant agreement no. 606862 (FCHROMA)and ERC grant agreement no. 291058 (CHROMPHYS). IRIS is a NASA small explorer mission developed and operated by LMSAL with mission operations executed at NASA Ames Research center and major contributions to downlink communications funded by ESA and the Norwegian Space Centre.

\section{Appendix A \\ The Balmer Jump Ratio, Upper Photospheric Heating, and Red Optical Continuum Emission in the 5F11 Model}

An important property of optically thin hydrogen recombination emission is a large ratio of NUV to optical excess continuum intensity (Kowalski et al. 2015b), which is evident in the continuum spectrum of the 5F11 model in Figure 10. The ROSA instrument (Jess et al. 2008) employs two custom continuum filters at $\lambda=3500$ and $4170 \AA$ which can provide measurements of the Balmer jump ratio as done for flares on other stars with the high speed camera ULTRACAM (Kowalski et al. 2016). For the 5F11 model the Balmer jump ratio $\left(F_{3500} / F_{4170}\right)$ of the excess continuum emission is $\sim 9$ in the rise phase and peak of Figure 7, which is consistent with an optically thin hydrogen recombination spectrum. These Balmer jump ratio measurements are not available for this flare, so we also calculate a ratio of excess C2826 to excess red optical continuum emission (C6173) near in wavelength $(\lambda=6173 \AA)$ to $S D O / \mathrm{HMI}$, which is often used as a proxy for the optical continuum intensity in solar flares (e.g., Kuhar et al. 2016). Using the data from Table 2 of Kleint et al. (2016), this ratio is approximately 5 for the brightest footpoints observed with both instruments (e.g., BFP1 and BFP2). In the 5F11 model, the $\mathrm{C} 2826 / \mathrm{C} 6173$ ratio is 5 at $t=1.8 \mathrm{~s}$ and $t=3.97 \mathrm{~s}$, and is generally consistent with these observational constraints. ${ }^{17}$

The temperature evolution of the 5F11 atmosphere at a representative height in the pre-flare upper photosphere

\footnotetext{
17 We have assumed that the $S D O / \mathrm{HMI}$ flare contrast is spatially resolved in this flare; using the IRIS data, the BFP1 and BFP2 footpoints are just resolved at the resolution of HMI (see Section 7).
}

$(z=140 \mathrm{~km})$ is shown in Figure 7 on the right axis with values ranging from $T=4750 \mathrm{~K}$ to $T=5680 \mathrm{~K}$. The height of $z \sim 140 \mathrm{~km}$ in the $5 \mathrm{~F} 11$ model is at the same column mass $\left(0.25 \mathrm{~g} \mathrm{~cm}^{-2}\right)$ as $z \sim 350 \mathrm{~km}$ in the VAL3C used in the phenomenological modeling studies of this flare with the RH code (Judge et al. 2014; Kleint et al. 2016). In the 5F11 model, the temperature increase at $z \lesssim 500 \mathrm{~km}$ is due to the absorption of Balmer continuum photons produced in the stationary flare layers and CC (radiative backwarming; Machado et al. 1989), as in the RHD models of Allred et al. $(2005,2006)$ and Cheng et al. (2010). The 5F11 model predictions of the upperphotospheric heating from NUV backwarming are in general agreement with the increase in temperature in the upper photosphere from the phenomenological models of Kleint et al. (2016).

The evolution of the emergent intensity contrast $\left(\frac{I-I_{\text {pre }}}{I_{\text {pre }}}\right)$ at $\lambda=6173 \AA$ is also shown in Figure 7. A maximum of $\sim 65 \%$ $70 \%$ contrast is achieved at $\sim 5 \mathrm{~s}$ while slightly increasing after this time for the extended heating run. The maximum contrast is several times larger than the peak values in this region of the SOL2014-03-29T17:48 flare (15\%; Kleint et al. 2016), but the contrast is only $\sim 25 \%$ at $1.8 \mathrm{~s}$ in the $5 \mathrm{~F} 11$.

Following the NUV continuum emissivity analysis in Section 5.5 , we calculate that $\sim 75 \%$ of the excess red optical (C6173) continuum intensity in the first four seconds of the $5 \mathrm{~F} 11$ originates from the the $\mathrm{CC}$ (and stationary flare layers) at $z>744 \mathrm{~km}$ (Table 3). Most of this emission is optically thin Paschen recombination radiation. The remaining $\sim 25 \%$ of the excess $\mathrm{C} 6173$ intensity originates from $\mathrm{H}^{-}$emission below the stationary flare layers at $z \sim 200-744 \mathrm{~km}$; the upper photosphere at $z \lesssim 200 \mathrm{~km}$ does not produce large $\mathrm{H}^{-}$emissivity until it heats more than the $\Delta T \sim 300 \mathrm{~K}$ that occurs within the first four seconds. In contrast to the NUV, the total optical continuum emissivity at $z>200 \mathrm{~km}$ has non-negligible amounts of hydrogen free-free emission ${ }^{18}$ at higher temperatures near $T \sim 25,000 \mathrm{~K}$ and $\mathrm{H}^{-}$bound-free emission from the cooler layers, which are heated by the beam and backwarming at $z<744 \mathrm{~km}$.

We extend the red optical continuum analysis to $t=15 \mathrm{~s}$ in the extended 5F11 heating model which gives insight into the difference in the time evolution of the excess C2826 and the C6173 contrast in Figure 7. The temperature of the $\mathrm{CC}$ at $15 \mathrm{~s}$ $\left(T=7500 \mathrm{~K}\right.$; Figure 9) produces increasingly strong $\mathrm{H}^{-}$ emission at $\lambda=6173 \AA$ which is comparable to the Paschen recombination emission in the $\mathrm{CC}$ at this time. Due to the temperature increase in the upper photosphere, $\Delta T_{\text {upperphot }} \sim 900$ $\mathrm{K}$, the C6713 emissivity from lower heights $(z<500 \mathrm{~km})$ is nearly comparable to the emission from the CC at $t=15 \mathrm{~s}$.

The excess red optical light curve (Figure 7) is rather flat at later times in the extended 5F11 simulation and the NUV light curve decreases. Therefore, the Balmer jump ratio decreases over time. By $15 \mathrm{~s}$, the amount of height-integrated hydrogen NUV (Balmer) b-f emissivity in the CC and stationary flare layers has decreased. In the NUV, the increased amount of $\mathrm{H}^{-}$ emission from the upper photosphere is insignificant compared to the (bright) Balmer continuum emission and thus the light curve is dominated by the evolution of the NUV continuum emission in the CC. The NUV emergent continuum intensity decreases after $5 \mathrm{~s}$ due to a small physical depth range

\footnotetext{
18 At $t=1.8 \mathrm{~s}$ there are equal amounts of hydrogen free-free and hydrogen bound-free red optical emissivity in the $\mathrm{CC}$.
} 
( $\Delta z \sim 25 \mathrm{~km}$ at $15 \mathrm{~s}$, compared to $160 \mathrm{~km}$ at $t=3.97 \mathrm{~s}$ ). The Balmer jump ratio of the excess spectrum decreases from 9 at $t=3.97 \mathrm{~s}$ to $\sim 5-6$ at $15 \mathrm{~s}$ in the extended $5 \mathrm{~F} 11$ heating simulation; the C2826/C6173 continuum ratio decreases from 5 to $\sim 3$. The decrease in the ratio of excess NUV to optical continuum intensity is due to the smaller physical depth range of Balmer continuum intensity in the NUV and the increasing $\mathrm{H}^{-}$optical emissivity from the upper photosphere which heats to $T \sim 5700 \mathrm{~K}$ and from the CC which cools below $T \sim 8000 \mathrm{~K}$.

In summary, upper photospheric heating of $\Delta T \sim 900 \mathrm{~K}$ occurs in the 5F11 model but is delayed with respect to the NUV continuum emission. The heating in the upper photosphere is caused by radiative backwarming by photons in the Balmer continuum range as in previous RHD models with lower beam fluxes (Allred et al. 2005). The temperature increase inferred from the phenomenological modeling of the IRIS NUV data during this flare in Kleint et al. (2016), Judge et al. (2014) is generally consistent with the 5F11 model backwarming effects. Upper photospheric $(z \sim 140 \mathrm{~km})$ heating by $\Delta T \sim 300 \mathrm{~K}$ that occurs within the first four seconds in the 5F11 but the majority of the excess red optical continuum intensity in the early times of the heating is from optically thin Paschen recombination emission in the $\mathrm{CC}$ and stationary flare layers. An increase in the $\mathrm{H}^{-}$emission occurs in all layers from the upper photosphere to the chromospheric condensation as the upper photosphere heats up and the condensation cools. A predominantly optically thin hydrogen recombination spectrum in the early phase predicts a large Balmer jump ratio, and the increasing $\mathrm{H}^{-}$emission decreases the ratio for extended beam heating.

\section{Appendix B \\ Constraints on Hot $(T \sim 9000 \mathrm{~K})$ Blackbody-like Emission at Blue Optical Wavelengths}

Kretzschmar (2011) measured an optical color temperature of $T \sim 9000 \mathrm{~K}$ in Sun-as-a-Star narrowband continuum data in blue $(4020 \AA)$, green $(5000 \AA)$, and red $(8620 \AA)$ filters from the VIRGO/SPM instrument (on the $\mathrm{SOHO}$ spacecraft) for superposed flares of GOES class $\mathrm{C}, \mathrm{M}$, and X-class in Solar Cycle 23.

Kleint et al. (2016) combined the IRIS NUV intensity values with HMI $(\lambda \sim 6173 \AA)$ and $\operatorname{IR~}(\lambda \sim 10832 \AA)$ intensity measurements to constrain the coarse properties of the whitelight spectral energy distribution (for the footpoints observed with spectra, including BFP1 and BFP2) in the SOL2014-0329T17:48 flare. A blackbody with $T \sim 6300 \mathrm{~K}$ could fit the optical and IR data, and a Balmer continuum emission component was necessary to account for the IRIS NUV continuum enhancement (see Kowalski et al. 2010, 2013, for a similar conclusion using spectra of dMe flares). Moreover, Kleint et al. (2016) noted that subtracting a pre-flare blackbody $(T=5770 \mathrm{~K})$ from the flare blackbody $(T=6300 \mathrm{~K})$ results in an optical color temperature of $T \sim 9000 \mathrm{~K}$ for the SOL201403-29T17:48 flare, putting the results for this flare in line with the results from the Sun-as-a-star analysis of Kretzschmar (2011).

We repeat the calculations (L. Kleint 2016, private communication) using the data from Kretzschmar (2011) while adjusting for the fraction of the solar surface no longer emitting at pre-flare values during the flare (Equation 3 of Kowalski et al. 2016) for a direct comparison to a blackbody flare model.
The high-thresh area in the SJI2832 images for the SOL201403-29T17:48 flare is $\sim 10 \operatorname{arcsec}^{2}$ (Section 3), which is also the typical area of white-light emission sources in TRACE/WL data (L. Fletcher 2015, priv. communication; Hudson et al. 2006; Fletcher et al. 2007). Assuming this area for the optical flare emission observed with VIRGO/SPM, the color temperature range that characterizes the peaks of the average $\mathrm{X}$-class and average M-class flares becomes $T \sim 8000-8500$ $\mathrm{K}$, or about $1000 \mathrm{~K}$ less than the values ${ }^{19}$ in Kretzschmar (2011). However, the high-thresh (where $I_{\lambda, \text { excess }} \geqslant 3 \times 10^{6} \mathrm{erg} \mathrm{cm}^{-2} \mathrm{~s}^{-1}$ s.r. $^{-1} \AA^{-1}$ ) excess specific luminosity accounts for only $\sim 1 / 3$ of the low-thresh excess specific luminosity in the impulsive phase and thus the emission from the high-thresh area is not likely to dominate a Sun-as-a-star measurement as would be observed with VIRGO/SPM; a larger area may be closer to the relevant area to use to interpret the measurements in Kretzschmar (2011). If we use the area of the low-thresh region for the SJI 2832 images $\left(\sim 100 \operatorname{arcsec}^{2}\right)$, then the color temperature range that characterizes the peaks of the VIRGO/SPM flares becomes $\sim 7000 \mathrm{~K}$ (or less for larger areas), which is closer to the observed color temperature in this flare (Kleint et al. 2016). The assumptions for these calculations include that the area emits at the standard irradiance value in each filter before the flare occurs. If this area were brighter than the standard value before the flare, then the inferred color temperature decreases; if this area were dimmer the inferred color temperature increases.

The spectra of BFP1 and BFP2 at 17:46:08+75 s and $17: 46: 24+75 \mathrm{~s}$, respectively, in Figure 3 are in the decaying phase of these sources and show NUV continuum emission with a C2826 excess of $\sim 0.25-0.5 \times 10^{6} \mathrm{erg} \mathrm{cm}^{-2} \mathrm{~s}^{-1}$ s.r. ${ }^{-1} \AA^{-1}$. This range is less than the low thresh criterion for SJI 2832 emission $\left(I_{\lambda, \text { excess }}>0.6 \times 10^{6} \mathrm{erg} \mathrm{cm}^{-2} \mathrm{~s}^{-1}\right.$ s.r. ${ }^{-1} \AA^{-1}$; Section 3). Accounting for some contribution from emission lines in SJI 2832, faint NUV continuum intensity would be expected to contribute to the areas corresponding to the low-thresh value. However, the Balmer continuum-emitting (NUV) areas have been inferred to be an order of magnitude larger than optical-continuum emitting areas in dMe flares (Kowalski et al. 2010), and the optical and IR continuum source size in an X-class solar flare has been found to vary as a function of wavelength (Xu et al. 2012). High spatial resolution measurements of solar flare optical and NUV areal measurements would clarify the source area to use when interpreting the data from Kretzschmar (2011).

We measure the color temperature of the blue-to-red optical continuum in the 5F11 model in Figure 10 for comparison to the observational constraints in Kleint et al. (2016). In the first $4 \mathrm{~s}$, the color temperature from $\lambda=4020 \AA$ to $5000 \AA$ ranges between $T=6600-6900 \mathrm{~K}$, which is comparable to the observed color temperature $(6300 \mathrm{~K})$ in the SOL2014-0329T17:48 flare. The color temperature during the 5F11 flare heating is lower than the color temperature $(T \sim 7100 \mathrm{~K})$ at $t=0 \mathrm{~s}$ because the RADYN model ${ }^{20}$ does not include line haze opacity at blue and violet wavelengths (Vernazza et al. 1976). In the 5F11 model, the lower color temperature of the blue optical wavelength range during the flare results

\footnotetext{
${ }^{19}$ For the 2003 October 28 flare considered in Kretzschmar (2011) with an area that has been directly measured to be $130 \operatorname{arcsec}^{2}$, we obtain a refined estimate of $7900 \mathrm{~K}$.

${ }^{20}$ At $t=0 \mathrm{~s}, T_{\mathrm{rad}}=5740 \mathrm{~K}$ and $T_{\mathrm{rad}}=6040 \mathrm{~K}$ at $\lambda=5000 \AA$ thus giving a higher color temperature.
} 
because the flare emission is dominated by optically thin Paschen recombination radiation (with a smaller contribution from hydrogen free-free emission) as found for the red optical continuum intensity in Appendix A.

We use the high spatial resolution of the IRIS SJI 2832 data to characterize the largest possible radiation temperature for the brightest pixels of BK2830 in the SOL2014-03-29T17:48 flare, which corresponds to $T_{\mathrm{rad}} \approx 7560 \mathrm{~K}$. The blackbody radiation temperature of $\sim 7560 \mathrm{~K}$ is close to the refined range of $T=8000-8500 \mathrm{~K}$ for the VIRGO/SPM data of Kretzschmar (2011) using the smaller estimate for the area. However, the 5F11 model provides an alternative explanation (Section 6) for the brightest NUV flare pixels in the SJI 2832 data of the SOL2014-03-29T17:48 flare as a combination of Fe II emission lines and hydrogen Balmer recombination radiation from low optical depth. For the brightest pixels with spectra and slit jaw data in the impulsive phase, a moderately hot $(T \gtrsim 8000-9000 \mathrm{~K})$ blackbody-like component is thus not necessary to explain the IRIS data. However, due to the relatively low time sampling of the NUV spectral and SJI data relative to the impulsive phase duration $(\sim 120 \mathrm{~s})$, the constraints on the formation of a hot blackbody-like spectrum in a given pixel for this flare are limited. We note that the nonthermal power for the SOL2014-03-29T17:48 flare is low $\left(8 \times 10^{27} \mathrm{erg} \mathrm{s}^{-1}\right.$ Kleint et al. 2016) compared to the nonthermal power inferred for some other flares $\left(\sim 10^{29} \mathrm{erg}\right.$ $\mathrm{s}^{-1}$; Matthews et al. 2003; Fletcher et al. 2007; Milligan et al. 2014). The super-posed epoch analysis of data from Kretzschmar (2011) may be biased toward the brighter whitelight solar flares (Kerr \& Fletcher 2014), and brighter whitelight emission generally occurs in flares with higher electron power above $50 \mathrm{keV}$ (Kuhar et al. 2016).

\section{Appendix C \\ Comparison of 5F11 and F13 RHD Models}

In the impulsive phase of impulsive-type flares from active $\mathrm{M}$ dwarf stars, the NUV and blue optical continuum distribution often exhibits a color temperature of $T \sim$ 10,000-12,000 K, with a small Balmer jump in emission (Hawley \& Pettersen 1991; Kowalski et al. 2013, 2016). Due to a low surface flux of an $\mathrm{M}$ dwarf in quiescence, subtracting an $\mathrm{M}$ dwarf pre-flare spectrum from a flare observation (to infer a color temperature) does not artificially produce a hot continuum in the blue and NUV as is possible for the Sun as discussed in Kleint et al. (2016).

A hot blackbody-like continuum spectrum in the NUV and optical has been produced recently in an RHD simulation of the response of an $\mathrm{M}$ dwarf atmosphere to an F13 nonthermal electron beam (Kowalski et al. 2015b, 2016), which also produces white-light emitting $\mathrm{CC}$ and stationary flare layers. In the $5 \mathrm{~F} 11$ electron beam model, the Balmer continuum emission originates over a large physical depth range $(\Delta z=50-390 \mathrm{~km}$; Table 3), which is not strongly wavelength dependent, due to the low optical depth in the CC. In the F13 simulation, the Balmer continuum emission originates over a much smaller physical depth range of $\sim 1 \mathrm{~km}$ (Kowalski 2016) ${ }^{21}$ due to the larger density and optical depth in the CC. In the F13 model, the physical depth range is strongly wavelength dependent, and

\footnotetext{
${ }^{21}$ Using $\Delta z$ defined as we have in this paper; Kowalski et al. (2015b) used the FHWM of the contribution function as an indication of the physical depth range. Using the FWHM of the contribution function at $t=3.97 \mathrm{~s}$ for the $5 \mathrm{~F} 11$ model, the physical depth range of $\mathrm{C} 2826$ continuum intensity is $3.5 \mathrm{~km}$.
}

only the blue optical photons $(\lambda \sim 4300 \AA)$ have an optical depth that is low enough to escape from the stationary flare layers. Thus, we predict that optically thick lines should not exhibit an emission component at $\lambda_{\text {rest }}$ for flare atmospheres with CC's that exhibit large values of the optical depth at continuum wavelengths, such as a hot $(T \geqslant 9000 \mathrm{~K})$ blackbody-like spectrum.

\section{References}

Abbett, W. P., \& Hawley, S. L. 1999, ApJ, 521, 906

Allred, J. C., Hawley, S. L., Abbett, W. P., \& Carlsson, M. 2005, ApJ, 630, 573 Allred, J. C., Hawley, S. L., Abbett, W. P., \& Carlsson, M. 2006, ApJ, 644, 484 Allred, J. C., Kowalski, A. F., \& Carlsson, M. 2015, ApJ, 809, 104

Avrett, E. H., Machado, M. E., \& Kurucz, R. L. 1986, in The Lower Atmosphere of Solar Flares, ed. D. F. Neidig (Sunspot, NM: National Solar Observatory), 216

Battaglia, M., \& Benz, A. O. 2006, A\&A, 456, 751

Battaglia, M., \& Benz, A. O. 2008, A\&A, 487, 337

Battaglia, M., Kleint, L., Krucker, S., \& Graham, D. 2015, ApJ, 813, 113

Battaglia, M., Kontar, E. P., Fletcher, L., \& MacKinnon, A. L. 2012, ApJ, 752, 4

Boyer, R., Sotirovsky, P., Machado, M. E., \& Rust, D. M. 1985, SoPh, 98, 255 Brannon, S. R., Longcope, D. W., \& Qiu, J. 2015, ApJ, 810, 4

Brown, J. C. 1971, SoPh, 18, 489

Canfield, R. C., \& Gayley, K. G. 1987, ApJ, 322, 999

Canfield, R. C., Penn, M. J., Wulser, J.-P., \& Kiplinger, A. L. 1990, ApJ, 363,318

Carlsson, M. 1998, in Space Solar Physics: Theoretical and Observational Issues in the Context of the SOHO Mission, ed. J. C. Vial, K. Bocchialini, \& P. Boumier (Berlin: Springer), 163

Carlsson, M., Leenaarts, J., \& De Pontieu, B. 2015, ApJL, 809, L30

Carlsson, M., \& Stein, R. F. 1992, ApJL, 397, L59

Carlsson, M., \& Stein, R. F. 1994, in Chromospheric Dynamics, ed. M. Carlsson (Oslo: University), 47

Carlsson, M., \& Stein, R. F. 1995, ApJL, 440, L29

Carlsson, M., \& Stein, R. F. 1997, ApJ, 481, 500

Carlsson, M., \& Stein, R. F. 2002, ApJ, 572, 626

Cauzzi, G., Falchi, A., Falciani, R., \& Smaldone, L. A. 1996, A\&A, 306, 625 Cavallini, F. 2006, SoPh, 236, 415

Cheng, J. X., Ding, M. D., \& Carlsson, M. 2010, ApJ, 711, 185

De Pontieu, B., Title, A. M., Lemen, J. R., et al. 2014, SoPh, 289, 2733

Dickson, E. C. M., \& Kontar, E. P. 2013, SoPh, 284, 405

Donati-Falchi, A., Falciani, R., \& Smaldone, L. A. 1985, A\&A, 152, 165

Donati-Falchi, A., Smaldone, L. A., \& Falciani, R. 1984, A\&A, 131, 256

Dorfi, E. A., \& Drury, L. O. 1987, JCoPh, 69, 175

Emslie, A. G. 1978, ApJ, 224, 241

Emslie, A. G. 1980, ApJ, 235, 1055

Emslie, A. G., \& Nagai, F. 1985, ApJ, 288, 779

Falchi, A., \& Mauas, P. J. D. 2002, A\&A, 387, 678

Fisher, G. H. 1989, ApJ, 346, 1019

Fisher, G. H., Canfield, R. C., \& McClymont, A. N. 1985, ApJ, 289, 434

Fletcher, L., Hannah, I. G., Hudson, H. S., \& Metcalf, T. R. 2007, ApJ, 656, 1187

Fletcher, L., \& Hudson, H. S. 2002, SoPh, 210, 307

Fletcher, L., \& Hudson, H. S. 2008, ApJ, 675, 1645

Fuhrmeister, B., Liefke, C., Schmitt, J. H. M. M., \& Reiners, A. 2008, A\&A, 487, 293

Gan, W. Q., Rieger, E., Zhang, H. Q., \& Fang, C. 1992, ApJ, 397, 694

Graham, D. R., \& Cauzzi, G. 2015, ApJL, 807, L22

Grigis, P. C., \& Benz, A. O. 2004, A\&A, 426, 1093

Gritsyk, P. A., \& Somov, B. V. 2014, AstL, 40, 499

Halenka, J., \& Grabowski, B. 1984, A\&AS, 57, 43

Hannah, I. G., Kontar, E. P., \& Sirenko, O. K. 2009, ApJL, 707, L45

Hawley, S. L., Allred, J. C., Johns-Krull, C. M., et al. 2003, ApJ, 597, 535

Hawley, S. L., \& Fisher, G. H. 1992, ApJS, 78, 565

Hawley, S. L., \& Pettersen, B. R. 1991, ApJ, 378, 725

Hawley, S. L., Walkowicz, L. M., Allred, J. C., \& Valenti, J. A. 2007, PASP, 119,67

Heinzel, P., Kašparová, J., Varady, M., Karlický, M., \& Moravec, Z. 2016, in IAU Symp. 320, Solar and Stellar Flares and their Effects on Planets, ed. A. G. Kosovichev, S. L. Hawley, \& P. Heinzel (Cambridge: Cambridge Univ. Press), 233

Heinzel, P., \& Kleint, L. 2014, ApJL, 794, L23 
Hiei, E. 1982, SoPh, 80, 113

Holman, G. D. 1985, ApJ, 293, 584

Holman, G. D. 2012, ApJ, 745, 52

Holman, G. D., Sui, L., Schwartz, R. A., \& Emslie, A. G. 2003, ApJL, 595, L97

Hudson, H. S. 1972, SoPh, 24, 414

Hudson, H. S., Acton, L. W., Hirayama, T., \& Uchida, Y. 1992, PASJ, 44, L77

Hudson, H. S., Wolfson, C. J., \& Metcalf, T. R. 2006, SoPh, 234, 79

Ichimoto, K., \& Kurokawa, H. 1984, SoPh, 93, 105

Isobe, H., Kubo, M., Minoshima, T., et al. 2007, PASJ, 59, 807

Jess, D. B., Mathioudakis, M., Crockett, P. J., \& Keenan, F. P. 2008, ApJL, 688, L119

Jing, J., Xu, Y., Cao, W., et al. 2016, NatSR, 6, 24319

Judge, P. G., Jordan, C., \& Feldman, U. 1992, ApJ, 384, 613

Judge, P. G., Kleint, L., Donea, A., Sainz Dalda, A., \& Fletcher, L. 2014, ApJ, 796, 85

Kašparová, J., Varady, M., Heinzel, P., Karlický, M., \& Moravec, Z. 2009, A\&A, 499, 923

Kennedy, M. B., Milligan, R. O., Allred, J. C., Mathioudakis, M., \& Keenan, F. P. 2015, A\&A, 578, A72

Kerr, G. S., \& Fletcher, L. 2014, ApJ, 783, 98

Kerr, G. S., Simões, P. J. A., Qiu, J., \& Fletcher, L. 2015, A\&A, 582, A50

Kleint, L., Battaglia, M., Reardon, K., et al. 2015, ApJ, 806, 9

Kleint, L., Heinzel, P., Judge, P., \& Krucker, S. 2016, ApJ, 816, 88

Kowalski, A. F. 2016, in IAU Symp. 320, Solar and Stellar Flares and their Effects on Planets, ed. A. G. Kosovichev \& P. Heinzel (Cambridge: Cambridge Univ. Press), 259

Kowalski, A. F., Cauzzi, G., \& Fletcher, L. 2015a, ApJ, 798, 107

Kowalski, A. F., Hawley, S. L., Carlsson, M., et al. 2015b, SoPh, 290, 3487

Kowalski, A. F., Hawley, S. L., Holtzman, J. A., Wisniewski, J. P., \& Hilton, E. J. 2010, ApJL, 714, L98

Kowalski, A. F., Hawley, S. L., Wisniewski, J. P., et al. 2013, ApJS, 207, 15

Kowalski, A. F., Mathioudakis, M., Hawley, S. L., et al. 2016, ApJ, 820, 95

Kretzschmar, M. 2011, A\&A, 530, A84

Krucker, S., Hudson, H. S., Glesener, L., et al. 2010, ApJ, 714, 1108

Krucker, S., Hudson, H. S., Jeffrey, N. L. S., et al. 2011, ApJ, 739, 96

Kuhar, M., Krucker, S., Martínez Oliveros, J. C., et al. 2016, ApJ, 816, 6

Kuridze, D., Mathioudakis, M., Simões, P. J. A., et al. 2015, ApJ, 813, 125

Lee, K. W., Büchner, J., \& Elkina, N. 2008, A\&A, 478, 889

Leenaarts, J., Pereira, T., \& Uitenbroek, H. 2012, A\&A, 543, A109

Leenaarts, J., Pereira, T. M. D., Carlsson, M., Uitenbroek, H., \& De Pontieu, B. 2013a, ApJ, 772, 89

Leenaarts, J., Pereira, T. M. D., Carlsson, M., Uitenbroek, H., \& De Pontieu, B. 2013b, ApJ, 772, 90

Li, T. C., Drake, J. F., \& Swisdak, M. 2014, ApJ, 793, 7

Lin, R. P., Dennis, B. R., Hurford, G. J., et al. 2002, SoPh, 210, 3

Liu, W., Heinzel, P., Kleint, L., \& Kašparová, J. 2015, SoPh, 290, 3525

Liu, W., Petrosian, V., \& Mariska, J. T. 2009, ApJ, 702, 1553

Livshits, M. A., Badalian, O. G., Kosovichev, A. G., \& Katsova, M. M. 1981, SoPh, 73, 269

Machado, M. E., Avrett, E. H., Vernazza, J. E., \& Noyes, R. W. 1980, ApJ, 242,336
Machado, M. E., Emslie, A. G., \& Avrett, E. H. 1989, SoPh, 124, 303

Magain, P. 1986, A\&A, 163, 135

Martínez Oliveros, J.-C., Hudson, H. S., Hurford, G. J., et al. 2012, ApJL, 753, L26

Matthews, S. A., Harra, L. K., Zharkov, S., \& Green, L. M. 2015, ApJ, 812, 35

Matthews, S. A., van Driel-Gesztelyi, L., Hudson, H. S., \& Nitta, N. V. 2003, A\&A, 409, 1107

Mauas, P. J. D., \& Gomez, D. O. 1997, ApJ, 483, 496

Mauas, P. J. D., Machado, M. E., \& Avrett, E. H. 1990, ApJ, 360, 715

McTiernan, J. M., \& Petrosian, V. 1990, ApJ, 359, 524

Metcalf, T. R., Alexander, D., Hudson, H. S., \& Longcope, D. W. 2003, ApJ, 595,483

Mihalas, D. 1970, Stellar Atmospheres

Mihalas, D. 1978, Stellar Atmospheres (2nd ed.; San Francisco: Freeman)

Milligan, R. O., Kerr, G. S., Dennis, B. R., et al. 2014, ApJ, 793, 70

Nave, G., \& Johansson, S. 2013, ApJS, 204, 1

Nave, G., Johansson, S., Learner, R. C. M., Thorne, A. P., \& Brault, J. W 1994, ApJS, 94, 221

Neidig, D. F. 1983, SoPh, 85, 285

Neidig, D. F. 1989, SoPh, 121, 261

Neidig, D. F., \& Wiborg, P. H., Jr. 1984, SoPh, 92, 217

Penn, M., Krucker, S., Hudson, H., et al. 2016, ApJL, 819, L30

Petrosian, V., \& Liu, S. 2004, ApJ, 610, 550

Potts, H., Hudson, H., Fletcher, L., \& Diver, D. 2010, ApJ, 722, 1514

Raassen, A. J. J., \& Uylings, P. H. M. 1998, JPhB, 31, 3137

Reep, J. W., Bradshaw, S. J., \& Holman, G. D. 2016, ApJ, 818, 44

Reep, J. W., \& Russell, A. J. B. 2016, ApJL, 818, L20

Ricchiazzi, P. J., \& Canfield, R. C. 1983, ApJ, 272, 739

Rubio da Costa, F., Kleint, L., Petrosian, V., Liu, W., \& Allred, J. C. 2016, ApJ, 827, 38

Rubio da Costa, F., Liu, W., Petrosian, V., \& Carlsson, M. 2015, ApJ, 813, 133

Russell, A. J. B., \& Fletcher, L. 2013, ApJ, 765, 81

Rust, D. M., \& Hegwer, F. 1975, SoPh, 40, 141

Rutten, R. J. 2003, Radiative Transfer in Stellar Atmospheres, Lecture Notes Utrecht University

Scharmer, G. B. 1981, ApJ, 249, 720

Scharmer, G. B., \& Carlsson, M. 1985, JCoPh, 59, 56

Sharykin, I. N., \& Kosovichev, A. G. 2014, ApJL, 788, L18

Sikström, C. M., Schultz-Johanning, M., Kock, M., et al. 1999, JPhB, 32, 5687

Simões, P. J. A., \& Kontar, E. P. 2013, A\&A, 551, A135

Uitenbroek, H. 2001, ApJ, 557, 389

van den Oord, G. H. J. 1990, A\&A, 234, 496

Vernazza, J. E., Avrett, E. H., \& Loeser, R. 1976, ApJS, 30, 1

Walkowicz, L. M., Johns-Krull, C. M., \& Hawley, S. L. 2008, ApJ, 677, 593

Watanabe, K., Shimizu, T., Masuda, S., Ichimoto, K., \& Ohno, M. 2013, ApJ, 776,123

Xu, Y., Cao, W., Jing, J., \& Wang, H. 2012, ApJL, 750, L7

Young, P. R., Tian, H., \& Jaeggli, S. 2015, ApJ, 799, 218

Zharkova, V. V., \& Gordovskyy, M. 2006, ApJ, 651, 553

Zhilyaev, B. E., Romanyuk, Y. O., Svyatogorov, O. A., et al. 2007, A\&A, 465,235 